\title{
3D field theories with Chern-Simons term for large $N$ in the Weyl gauge
}

\author{
Moshe Moshe ${ }^{a}$ and Jean Zinn-Justin ${ }^{b}$ \\ ${ }^{a}$ Department of Physics, Technion - Israel Institute of Technology, \\ Haifa, 32000 Israel \\ ${ }^{b}$ CEA/IRFU, Centre de Saclay, \\ 91191 Gif-sur-Yvette Cedex, France \\ E-mail: moshe@technion.ac.il, jean.zinn-justin@cea.fr
}

ABstract: Three dimensional, $\mathrm{U}(N)$ symmetric, field theory with fermion matter coupled to a topological Chern-Simons term, in the large $N$ limit is analyzed in details. We determine the conditions for the existence of a massless conformal invariant ground state as well as the conditions for a massive phase. We analyze the phase structure and calculate gauge invariant corelators comparing them in several cases to existing results. In addition to the non-critical explicitly broken scale invariance massive case we consider also a massive ground state where the scale symmetry is spontaneously broken. We show that such a phase appears only in the presence of a marginal deformation that is introduced by adding a certain scalar auxiliary field and discuss the fermion-boson dual mapping. The ground state contains in this case a massless $\mathrm{U}(N)$ singlet bound state goldstone boson- the dilaton whose properties are determined. We employ here the temporal gauge which is at variance with respect to past calculations using the light-cone gauge and thus, a check (though limited) of gauge independence is at hand. The large $N$ properties are determined by using a field integral formalism and the steepest descent method. The saddle point equations, which take here the form of integral equations for non-local fields, determine the mass gap and the dressed fermion propagator. Vertex functions are calculated at leading order in $1 / N$ as exact solutions of integral equations. From the vertex functions, we infer gauge invariant two-point correlation functions for scalar operators and a current. Indications about the consistency of the method are obtained by verifying that gauge-invariant quantities have a natural $O(3)$ covariant form. As a further verification, in several occasions, a few terms of the perturbative expansion are calculated and compared with the exact results in the appropriate order.

Keywords: 1/N Expansion, Spontaneous Symmetry Breaking, Chern-Simons Theories, Nonperturbative Effects

ARXIV EPRINT: 1410.0558v2 


\section{Contents}

1 Introduction 1

2 3D topological Chern-Simons action 3

2.1 Chiral anomaly, topology and instantons 3

2.2 Topological Chern-Simons action in 3D field theory 5

$\begin{array}{ll}2.3 & \text { The Chern-Simons action in the temporal gauge }\end{array}$

3 Chern-Simons gauge fields coupled to $U(N)$ fermions 9

$\begin{array}{llr}3.1 \text { Conventions } & 9\end{array}$

3.2 The CS action coupled to a gauge-invariant fermion action $\quad 10$

$\begin{array}{lll}3.3 & \text { Large } N \text { expansion: field integral formalism } & 11\end{array}$

$\begin{array}{ll}3.4 & \text { The large } N \text { action } \\ 3.5 & 12\end{array}$

$\begin{array}{ll}3.5 \text { Saddle point equations } & 12\end{array}$

$\begin{array}{lll}3.6 & \text { Solution } & 14\end{array}$

$\begin{array}{ll}3.7 & \text { The free energy density } \\ 3.8 & \text { The }\end{array}$

3.8 The fermion two-point function for $N$ large 17

$\begin{array}{ll}3.9 \text { Gauge-invariant observables } & 18\end{array}$

4 Perturbative calculations at large $N \quad 19$

$\begin{array}{lll}4.1 \text { The fermion two-point function at two-loop order } & 19\end{array}$

4.2 The ground state (or vacuum) energy up to three loops 20

5 Correlation functions involving $R=\bar{\psi} \cdot \psi$ at large $N \quad 22$

5.1 A simple transformation 22

5.2 Field integral formalism: expansion at the saddle point 22

5.3 The $\langle(\bar{\psi} \psi) \psi \bar{\psi}\rangle$ vertex function 24

5.4 The perturbative expansion of the $\langle(\bar{\psi} \psi) \psi \bar{\psi}\rangle$ vertex function at two loops $\quad 25$

$\begin{array}{ll}5.5 \text { Integral equation } & 29\end{array}$

5.6 Solution of the reduced integral equation 31

$6 \quad$ The $R$ two-point function $\quad 32$

$\begin{array}{lll}6.1 & \text { Perturbative calculations } & 32\end{array}$

$6.2 R$ two-point function and vertex three-point function 34

6.3 The exact $R$ two-point function at large $N \quad 36$

$\begin{array}{lll}7 & \text { Mass gap and critical coupling } & 36\end{array}$

8 The current two-point function $\quad 38$

$\begin{array}{ll}\text { 8.1 The }\left\langle J_{3} \psi \bar{\psi}\right\rangle \text { vertex } & 39\end{array}$

$\begin{array}{lll}8.2 & \text { Perturbative calculations } & 39\end{array}$

$\begin{array}{lll}8.3 & \text { Integral equation } & 40\end{array}$ 
8.4 From the $J_{3}$ vertex to the $J_{3}$ two-point function 40

8.5 The $J_{3}$ two-point function 41

8.6 The $R$ three-point function 43

9 Adding a deformation to the Chern-Simons fermion action 44

9.1 Large $N$ limit: gap equation and free energy 45

$\begin{array}{lll}9.2 & \text { Connected scalar two-point functions at zero momentum } & 47\end{array}$

$\begin{array}{lll}9.3 & \text { The generic } R \text { two-point function } & 49\end{array}$

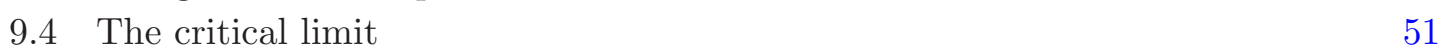

9.5 Situation with vanishing coefficient of $M^{2}$ in the gap equation 52

9.6 Spontaneously broken scale invariance in boson and fermion theories 53

$\begin{array}{ll}10 \text { Summary and conclusions } & 54\end{array}$

A Some explicit expressions $\quad \mathbf{5 5}$

A.1 The function $\Theta(\omega)$ and regularization $\quad 55$

A.2 The function $\mathcal{D}$ and the bound state problem $\quad 56$

$\begin{array}{ll}\text { B Relevant algebraic identities } & 57\end{array}$

B.1 Some elementary though useful identities $\quad 57$

$\begin{array}{lll}\text { B.2 Some relevant integrals } & 57\end{array}$

C The $\langle(\bar{\psi} \psi) \psi \bar{\psi}\rangle$ vertex: two-loop calculations $\quad 61$

$\begin{array}{ll}\text { C.1 Perturbative calculations } & 61\end{array}$

$\begin{array}{lc}\text { D The } J_{3} \text { vertex function at two loops } & 63\end{array}$

E The $R$ two-point function $\quad \mathbf{6 4}$

$\begin{array}{lll}\text { E.1 The } R \text { two-point function at three loops } & 65\end{array}$

$\begin{array}{lll}\text { E.2 The } R \text { two-point function at four loops } & 67\end{array}$

$\begin{array}{lll}\text { F } & \text { Two-point } J_{3} \text { calculations } & 68\end{array}$

$\begin{array}{lll}\text { F.1 One-loop calculation } & 68\end{array}$

$\begin{array}{ll}\text { G Other current components and WT identities } & 70\end{array}$

\section{Introduction}

It has been conjectured [1-4] that AdS/CFT correspondence implies a close relation between the singlet sector of $O(N)$ and $\mathrm{U}(N)$ bosons vector theory in $d=3$ space-time dimensions at large $N$ and Vasiliev's higher spin gravity theory $[5,6]$ on $\mathrm{AdS}_{4}$. Computation of correlation functions of the higher spin gauge theory [7-10] strengthen this conjecture. Since the quantum completion of the gravitational side of this duality is not 
known, only tree level could be considered. The AdS/CFT correspondence in this case provides a testing ground of AdS/CFT ideas. The large $N$ limit of $O(N)_{\kappa}$ and $\mathrm{U}(N)_{\kappa}$ level $\kappa$ Chern-Simons gauge theories, at $N, \kappa \rightarrow \infty$ with a fixed 't Hooft coupling $\lambda=N / \kappa$, coupled to scalar and fermion matter fields in the fundamental representation in $\mathrm{d}=3 \mathrm{di}$ mensions were studied recently [11]-[16]. Since pure Chern-Simons gauge theories has no propagating degrees of freedom, only the matter fields in the fundamental representation provide the true independent canonical degrees of freedom in these $d=3$ theories.

$O(N)$ and $\mathrm{U}(N)$ symmetric field theories at large $N$ are reasonably well understood [17]. In the case of Chern-Simons matter theories in $d=3$ dimensions, the explicit calculations that were performed [11]-[16] shed extra light on the AdS/CFT correspondence. The original $O(N)$ model of $g\left(\phi^{2}\right)^{2}$ in [1] was also deformed to include a marginal interaction term $\sim \lambda_{6}\left(\phi^{2}\right)^{3}$. The massless conformal invariant phase was analysed either by a generalized Hubbard-Stratanovich method [11] or in perturbation theory [12]. It was conjectured that the gravity dual of these $3 \mathrm{D}$ theories on the boundary is a parity broken version of Vasiliev's higher spin theory $[5,6]$ on $\mathrm{AdS}_{4}$ in the bulk with a parity breaking parameter $\theta$ which depends on the 't Hooft coupling $\lambda=N / \kappa$. Supersymmetric extension of this idea was introduced in [13]. It has also been conjectured that there is a duality between the boson and fermion theories [14] since the theory of bosons and fermions in the fundamental representation of $\mathrm{U}(N)$ coupled to Chern-Simons gauge fields are dual to the same bulk gravity theory on $\mathrm{AdS}_{4}$. Calculation of the thermal free energy in large $N$ ChernSimons field coupled to fermions and bosons has further strengthened this duality [16]. The thermal free energy has been calculated also in [11].

It was stated in ref. [16] that the introduction of masses through spontaneous breaking of scale invariance [18-21] is an alternative at large $N$. Though at finite $N$ the breakdown of the scale symmetry is explicit, it is of order $\mathcal{O}\left(N^{-1}\right)$, namely the same order by which the AdS/CFT correspondence is approximated in the above mentioned conjectures. Therefore it was found appealing to further analyse the Chern-Simons matter theory with bosons [22] and fermions [23] with masses introduced through spontaneous breaking of scale invariance, which ensures the existence of a massive ground state but leaves the boundary $d=3$ theory conformal to order $\mathcal{O}\left(N^{-1}\right)$. The calculations mentioned above were done using the light cone gauge fixing for the Chern-Simons gauge theory.

In this article we analyse the large $N$ limit of three dimensional, $\mathrm{U}(N)$ symmetric, field theory with fermion matter coupled by the topological Chern-Simons gauge field. Our calculational framework differs from the above mentioned calculations as we are employing the $A_{3}=0$ gauge rather than the light-cone gauge. In the temporal gauge, as in the lightcone gauge, the Chern-Simons action is reduced to a quadratic form and allows integrating out the gauge field. The large $N$ properties are determined by using then a path integral formalism and the steepest descent method in Euclidean space-time. In section 2 we start with a short discussion of the origin and properties of the topological Chern-Simons action. In section 3, the detailed description is presented of the Chern-Simons gauge field in the $A_{3}=0$ gauge coupled to $\mathrm{U}(N)$ fermions in the fundamental representation. The large $N$ action is discussed and the saddle point equations are determined. The saddle point equations, which take here the form of integral equations for non-local fields are then 
solved. In particular, the fermion dressed two point function $\tilde{\Gamma}^{(2)}(p)$, the gap equation and the ground state energy in the limit $N \rightarrow \infty$ are calculated in details. in section 3 we also derive the gauge-independent correlators of $\bar{\psi}_{\alpha}(x) \psi_{\alpha}(x)$ two- and three-point functions at zero momentum. As mentioned, these results are obtained by calculating the relevant path integral at large $N$ and thus they are further checked in section 4 by calculating in perturbation theory the fermion propagator and the ground state energy up to two-loops and three-loops, respectively. In section 5 the vertex function $\tilde{\Gamma}^{1,2}\left(k ; p_{1}, p_{2}\right)$ is calculated at leading order as solutions of integral equations.

Using the exact expression of the vertex function given in section 5 , we compute in section 6 the exact expression for the two-point function of $R(x)=\frac{1}{N} \bar{\psi}(x) \cdot \psi(x)$. Several correlation functions involving the $J_{\mu}$ current are calculated in section 8. Clearly, all these quantities are gauge-independent and can thus be compared to results obtained in other gauges. Indications about the consistency of the method are obtained by verifying that gauge-invariant quantities have a natural $O(3)$ covariant form. As a verification, we calculate a few terms of the perturbative expansion of the same correlation functions. In section 9, we introduce a scalar field $\sigma(x)$ coupled to the fermions. This deformation is analogous to the triple-trace deformation $\lambda_{6} \phi^{6}(x)$ added to the Chern-Simons boson theory $[16,22]$. We show that when a certain relation between the couplings constants is satisfied, the model exhibits spontaneous breaking of scale invariance, again in clear analogy to the Cherns-Simons boson case [16, 22]. The dilaton massless Goldstone boson excitation is a $\mathrm{U}(N)$ singlet bound state represented by an effective field which, in the large $N$ limit, is proportional to to $\sigma(x)-\langle\sigma\rangle \propto \bar{\psi}(x) \cdot \psi(x)$. Summary and conclusions are found in section 10. Several technical points and useful relations are included in several appendices.

\section{3D topological Chern-Simons action}

We first shortly recall the origin of the 3D Chern-Simons action and its main properties.

\subsection{Chiral anomaly, topology and instantons}

We consider an Abelian axial current in the framework of a non-Abelian gauge theory in four Euclidean dimensions. The fermion fields transform non-trivially under a unitary gauge group $G$ and $\mathbf{A}_{\mu}$ is the corresponding gauge field. The fermion action reads

$$
\mathcal{S}(\bar{\psi}, \psi ; A)=-\int \mathrm{d}^{4} x \bar{\psi}(x) \not \mathbf{D} \psi(x)
$$

with the convention

$$
\mathbf{D}_{\mu}=\mathbf{1} \partial_{\mu}+\mathbf{A}_{\mu}(x), \not D=\not \partial+\not \mathbf{A},
$$

where $\mathbf{A}_{\mu}$ is an anti-hermitian matrix, and the curvature tensor

$$
\mathbf{F}_{\mu \nu}=\left[\mathbf{D}_{\mu}, \mathbf{D}_{\nu}\right]=\partial_{\mu} \mathbf{A}_{\nu}-\partial_{\nu} \mathbf{A}_{\mu}+\left[\mathbf{A}_{\mu}, \mathbf{A}_{\nu}\right] .
$$

In a gauge transformation represented by a unitary matrix $\mathbf{g}(x)$, the gauge field $\mathbf{A}_{\mu}$ and the Dirac operator become

$$
\mathbf{A}_{\mu}(x) \mapsto \mathbf{g}(x) \partial_{\mu} \mathbf{g}^{-1}(x)+\mathbf{g}(x) \mathbf{A}_{\mu}(x) \mathbf{g}^{-1}(x) \Rightarrow \not D \mapsto \mathbf{g}^{-1}(x) \not \mathbf{g}(x) .
$$


The axial current

$$
J_{\mu}^{5}(x)=i \bar{\psi}(x) \gamma_{5} \gamma_{\mu} \psi(x)
$$

is gauge invariant. The axial anomaly [24, 25] leads to a non-vanishing of the divergence of the axial current given by

$$
\left\langle\partial_{\lambda} J_{\lambda}^{5}(x)\right\rangle=-\frac{i}{16 \pi^{2}} \epsilon_{\mu \nu \rho \sigma} \operatorname{tr} \mathbf{F}_{\mu \nu} \mathbf{F}_{\rho \sigma} .
$$

From general arguments, one knows that the expression (2.5) must be a total derivative. Indeed, one verifies that

$$
\epsilon_{\mu \nu \rho \sigma} \operatorname{tr} \mathbf{F}_{\mu \nu} \mathbf{F}_{\rho \sigma}=4 \epsilon_{\mu \nu \rho \sigma} \partial_{\mu} \operatorname{tr}\left(\mathbf{A}_{\nu} \partial_{\rho} \mathbf{A}_{\sigma}+\frac{2}{3} \mathbf{A}_{\nu} \mathbf{A}_{\rho} \mathbf{A}_{\sigma}\right)
$$

Anomaly, topology and instantons. Gauge field configurations can be found that contribute to the chiral anomaly. An especially interesting example is provided by instantons, that is finite action solutions of Euclidean field equations.

General instanton solutions can be exhibited by considering only pure gauge theories and the gauge group $\mathrm{SU}(2)$, since for a Lie group containing $\mathrm{SU}(2)$ as a subgroup the instantons are those of the $\mathrm{SU}(2)$ subgroup.

Instantons can be classified by a topological charge

$$
Q\left(\mathbf{A}_{\mu}\right)=\frac{1}{32 \pi^{2}} \int \mathrm{d}^{4} x \mathbf{F}_{\mu \nu} \cdot \tilde{\mathbf{F}}_{\mu \nu} .
$$

The expression $Q\left(\mathbf{A}_{\mu}\right)$ is proportional to the integral of the chiral anomaly (2.5), here written in $\mathrm{SO}(3)$ notation.

We know that $\mathbf{F}_{\mu \nu} \cdot \tilde{\mathbf{F}}_{\mu \nu}$ is a pure divergence (eq. (2.6)). In $\mathrm{SO}(3)$ notation,

$$
\mathbf{F}_{\mu \nu} \cdot \tilde{\mathbf{F}}_{\mu \nu}=\partial_{\mu} V_{\mu}
$$

with

$$
V_{\mu}=2 \epsilon_{\mu \nu \rho \sigma}\left[\mathbf{A}_{\nu} \cdot \partial_{\rho} \mathbf{A}_{\sigma}+\frac{1}{3} \mathbf{A}_{\nu} \cdot\left(\mathbf{A}_{\rho} \times \mathbf{A}_{\sigma}\right)\right] .
$$

The integral thus depends only on the behaviour of the gauge field at large distances and its values are quantized.

Stokes theorem implies

$$
\int_{\mathcal{D}} \mathrm{d}^{4} x \partial_{\mu} V_{\mu}=\int_{\partial \mathcal{D}} \mathrm{d} \Omega \hat{n}_{\mu} V_{\mu}
$$

where $d \Omega$ is the measure on the boundary $\partial \mathcal{D}$ of the four-volume $\mathcal{D}$ and $\hat{n}_{\mu}$ the unit vector normal to $\partial \mathcal{D}$. We take for $\mathcal{D}$ a sphere of large radius $R$ and find for the topological charge

$$
Q\left(\mathbf{A}_{\mu}\right)=\frac{1}{32 \pi^{2}} \int \mathrm{d}^{4} x \operatorname{tr} \mathbf{F}_{\mu \nu} \cdot \tilde{\mathbf{F}}_{\mu \nu}=\frac{1}{32 \pi^{2}} R^{3} \int_{r=R} \mathrm{~d} \Omega \hat{n}_{\mu} V_{\mu},
$$

The finiteness of the gauge action implies that classical solutions must asymptotically become pure gauges. Since $\mathrm{SU}(2)$ is topologically equivalent to the sphere $S_{3}$, the pure 
gauge configurations on a sphere of large radius $|x|=R$ define a mapping from $S_{3}$ to $S_{3}$. Such mappings belong to different homotopy classes that are characterized by an integer called the winding number. Here, we identify the homotopy group $\pi_{3}\left(S_{3}\right)$, which again is isomorphic to the additive group of integers $\mathbb{Z}$.

Without explicit calculation, one knows from the analysis of the index of the Dirac operator, that the topological charge is an integer:

$$
Q\left(\mathbf{A}_{\mu}\right)=\frac{1}{32 \pi^{2}} \int \mathrm{d}^{4} x \mathbf{F}_{\mu \nu} \cdot \tilde{\mathbf{F}}_{\mu \nu}=n \in \mathbb{Z} .
$$

\subsection{Topological Chern-Simons action in 3D field theory}

We now study some applications to 3D field theory of the existence of topological terms involving gauge fields in 4D quantum field theory, as recalled in section 2.1. We first discuss the pure Chern-Simons action and, then, the Chern-Simons action coupled to fermion matter in the large $N$ limit.

We first consider the 3D Euclidean action in the form of a Chern-Simons (CS) term

$$
\begin{aligned}
& \mathcal{S}_{\mathrm{CS}}(\mathbf{A})=-\frac{i \theta}{8 \pi^{2}} \mathrm{CS}_{3}(\mathbf{A}) \text { with } \\
& \mathrm{CS}_{3}(\mathbf{A})=\epsilon_{\mu \nu \rho} \int \mathrm{d}^{3} x \operatorname{tr}\left[\mathbf{A}_{\mu}(x) \partial_{\nu} \mathbf{A}_{\rho}(x)+\frac{2}{3} \mathbf{A}_{\mu}(x) \mathbf{A}_{\nu}(x) \mathbf{A}_{\rho}(x)\right],
\end{aligned}
$$

where $\mathbf{A}_{\mu}$ is a gauge field associated with $\mathrm{U}(N)$ gauge transformations as defined by equation (2.4):

$$
\mathbf{A}_{\mu}(x) \mapsto \mathbf{A}_{\mu}^{g} \equiv \mathbf{g}(x) \partial_{\mu} \mathbf{g}^{-1}(x)+\mathbf{g}(x) \mathbf{A}_{\mu}(x) \mathbf{g}^{-1}(x) .
$$

The CS action is locally gauge invariant but globally gauge invariant only up to a constant.

Indeed, starting from four dimensions, using the relation (2.6) and Stokes theorem, one infers that the expression

$$
\int \mathrm{d} \sigma_{3} \hat{n}_{\mu} V_{\mu}
$$

where the $\hat{n}_{\mu}$ is the normal to a 3 -surface, $\mathrm{d} \sigma_{3}$ the corresponding surface element and

$$
V_{\mu}=-4 \epsilon_{\mu \nu \rho \sigma} \operatorname{tr}\left(\mathbf{A}_{\nu} \partial_{\rho} \mathbf{A}_{\sigma}+\frac{2}{3} \mathbf{A}_{\nu} \mathbf{A}_{\rho} \mathbf{A}_{\sigma}\right)
$$

is gauge invariant up to a constant that depends only on a topological index, a property explained in section 2.1. The general result can then be applied to three-dimensional flat space.

As a consequence, strict gauge invariance of the integral over gauge fields then implies

$$
\theta=2 \pi \kappa,
$$

where $\kappa$ is an integer. 
Chern-Simons term and gauge transformations. As an exercise, we directly verify how $\mathrm{CS}_{3}\left(\mathbf{A}_{\mu}\right)$ transforms in a gauge transformation. We first rewrite $\mathrm{CS}_{3}\left(\mathbf{A}_{\mu}\right)$ as

$$
\mathrm{CS}_{3}\left(\mathbf{A}_{\mu}\right)=\epsilon_{\mu \nu \rho} \int \mathrm{d}^{3} x \operatorname{tr}\left[\frac{1}{2} \mathbf{A}_{\mu}(x) \mathbf{F}_{\nu \rho}(x)-\frac{1}{3} \mathbf{A}_{\mu}(x) \mathbf{A}_{\nu}(x) \mathbf{A}_{\rho}(x)\right] .
$$

It is convenient to define

$$
\mathbf{B}_{\mu}(x)=-\partial_{\mu} \mathbf{g}^{-1}(x) \mathbf{g}(x)=\mathbf{g}^{-1}(x) \partial_{\mu} \mathbf{g}(x),
$$

in such a way that gauge transformations read

$$
\mathbf{A}_{\mu}^{g}=\mathbf{g}(x)\left[\mathbf{A}_{\mu}(x)-\mathbf{B}_{\mu}(x)\right] \mathbf{g}^{-1}(x) .
$$

Then,

$$
\partial_{\nu} \mathbf{B}_{\rho}(x)=-\mathbf{B}_{\nu}(x) \mathbf{B}_{\rho}(x)+\mathbf{g}^{-1}(x) \partial_{\nu} \partial_{\rho} \mathbf{g}(x) .
$$

For a pure gauge, $\mathrm{CS}_{3}$ becomes

$$
\mathrm{CS}_{3}(\mathbf{g})=\frac{1}{3} \epsilon_{\mu \nu \rho} \int \mathrm{d}^{3} x \operatorname{tr} \mathbf{B}_{\mu}(x) \mathbf{B}_{\nu}(x) \mathbf{B}_{\rho}(x) .
$$

The quantity,

$$
\mathcal{T}=\frac{1}{3} \epsilon_{\mu \nu \rho} \int \mathrm{d}^{3} x \operatorname{tr} \mathbf{B}_{\mu}(x) \mathbf{B}_{\nu}(x) \mathbf{B}_{\rho}(x) .
$$

is a topological term. Let us verify this statement directly. We set

$$
\mathbf{g} \mapsto \mathbf{g}\left(\mathbf{1}+\mathbf{t}+O\left\|\mathbf{t}^{2}\right\|\right) .
$$

Then,

$$
\delta \mathbf{B}_{\mu}(x)=\partial_{\mu} \mathbf{t}(x)+\left[\mathbf{B}_{\mu}(x), \mathbf{t}(x)\right] O\left\|\mathbf{t}^{2}\right\| .
$$

The variation of expression (2.14) is

$$
\delta \mathcal{T} \sim \epsilon_{\mu \nu \rho} \int \mathrm{d}^{3} x \operatorname{tr} \partial_{\mu} \mathbf{t}(x) \mathbf{B}_{\nu}(x) \mathbf{B}_{\rho}(x) .
$$

We integrate by parts. The integrated term vanishes because the manifold has no boundaries. Thus,

$$
\delta \mathcal{T} \sim \epsilon_{\mu \nu \rho} \int \mathrm{d}^{3} x \operatorname{trt}(x)\left[\mathbf{B}_{\mu}(x) \mathbf{B}_{\nu}(x) \mathbf{B}_{\rho}(x)+\mathbf{B}_{\nu}(x) \mathbf{B}_{\mu}(x) \mathbf{B}_{\rho}(x)\right]=0 .
$$

Therefore, the quantity $\mathcal{T}$ does not depend on local changes of $\mathbf{g}(x)$ but only on its global properties.

Using gauge transformations in the form (2.13), we find

$$
\begin{aligned}
\mathrm{CS}_{3}\left(\mathbf{A}_{\mu}^{g}\right)= & \epsilon_{\mu \nu \rho} \int \mathrm{d}^{3} x \operatorname{tr}\left\{\frac{1}{2}\left[\mathbf{A}_{\mu}(x)-\mathbf{B}_{\mu}(x)\right] \mathbf{F}_{\nu \rho}(x)\right. \\
& \left.-\frac{1}{3}\left[\mathbf{A}_{\mu}(x)-\mathbf{B}_{\mu}(x)\right]\left[\mathbf{A}_{\nu}(x)-\mathbf{B}_{\nu}(x)\right]\left[\mathbf{A}_{\rho}(x)-\mathbf{B}_{\rho}(x)\right]\right\} \\
= & \mathrm{CS}_{3}\left(\mathbf{A}_{\mu}\right)+\epsilon_{\mu \nu \rho} \int \mathrm{d}^{3} x \operatorname{tr}\left\{-\mathbf{B}_{\mu}(x)\left[\partial_{\nu} \mathbf{A}_{\rho}(x)+\mathbf{A}_{\nu}(x) \mathbf{A}_{\rho}(x)\right]\right. \\
& \left.+\mathbf{A}_{\mu}(x) \mathbf{A}_{\nu}(x) \mathbf{B}_{\rho}(x)-\mathbf{A}_{\mu}(x) \mathbf{B}_{\nu}(x) \mathbf{B}_{\rho}(x)\right\} \\
& +\frac{1}{3} \operatorname{tr} \mathbf{B}_{\mu}(x) \mathbf{B}_{\nu}(x) \mathbf{B}_{\rho}(x) .
\end{aligned}
$$


We integrate by parts,

$$
\begin{gathered}
-\int \mathrm{d}^{3} x \operatorname{tr} \mathbf{B}_{\mu}(x) \partial_{\nu} \mathbf{A}_{\rho}(x)=-\int \mathrm{d} x_{\mu} \wedge \mathrm{d} x_{\rho} \operatorname{tr} \mathbf{B}_{\mu}(x) \mathbf{A}_{\rho}(x) \\
+\int \mathrm{d}^{3} x \operatorname{tr}\left[-\mathbf{B}_{\nu}(x) \mathbf{B}_{\mu}(x)+\mathbf{g}^{-1}(x) \partial_{\nu} \partial_{\mu} \mathbf{g}(x)\right] \mathbf{A}_{\rho}(x) .
\end{gathered}
$$

The vanishing of the boundary term implies that $\mathbf{B}_{\mu}$ should vanish and, thus, asymptotically $\mathbf{g}(x)$ should go to a constant for $|x|$ large. Therefore, $\mathbb{R}^{3}$ should be given the topology of $S_{3}$, as in the discussion of section 2.1 .

Assuming this condition satisfied and expanding, we conclude

$$
\mathrm{CS}_{3}\left(\mathbf{A}_{\mu}^{g}\right)=\mathrm{CS}_{3}\left(\mathbf{A}_{\mu}\right)+\frac{1}{3} \epsilon_{\mu \nu \rho} \int \mathrm{d}^{3} x \operatorname{tr} \mathbf{B}_{\mu}(x) \mathbf{B}_{\nu}(x) \mathbf{B}_{\rho}(x) .
$$

The Chern-Simons changes by a quantity that depends on global properties of the gauge transformation. Its values have indeed been calculated in section 2.1.

The classical equation of motion in an external source. We add a source to the action and consider the new action

$$
\mathcal{S}(\mathbf{A}, \mathbf{J})=-i \frac{\kappa}{4 \pi} \mathrm{CS}_{3}(\mathbf{A})+\int \mathrm{d}^{3} x \operatorname{tr} \mathbf{J}_{\mu}(x) \mathbf{A}_{\mu}(x) .
$$

The equation of motion obtained by varying $\mathbf{A}_{\mu}$ in $\mathrm{CS}_{3}$ yields

$$
\frac{\delta \mathrm{CS}_{3}}{\delta \mathbf{A}_{\mu}}=\epsilon_{\mu \nu \rho} \mathbf{F}_{\nu \rho}
$$

and, thus,

$$
\mathbf{F}_{\mu \nu}=-i \frac{2 \pi}{\kappa} \epsilon_{\mu \nu \rho} \mathbf{J}_{\rho} \Rightarrow \mathbf{D}_{\mu} \mathbf{J}_{\mu}=0 .
$$

For $\mathbf{J}=0$, the stationary solutions are pure gauges. In general, the gauge field has to be coupled to a gauge-covariant conserved source.

\subsection{The Chern-Simons action in the temporal gauge}

In general, to be able to define integrals over gauge fields, gauge fixing is required. In a general gauge, this leads to the introduction of Faddeev-Popov ghosts and the solution of the quantum field theory in the large $N$ limit involves the summation of planar diagrams [26], a problem whose solution is only known in lower dimensions [27]. However, in the case of the Chern-Simons action, in some special gauges like the light-cone or axial gauges, the cubic interaction term in the CS action vanishes, and the integral over gauge fields can be performed explicitly.

In contrast to previous work that mainly uses the light-cone gauge, we choose the gauge $\mathbf{A}_{3}=0$. Since we use an Euclidean formalism, we may identify $x_{3}$ with the Euclidean time (and below occasionally use the notation $t \equiv x_{3}$ and $\omega \equiv p_{3}$ in the Fourier representation). The CS action reduces to the quadratic form

$$
\mathrm{CS}_{3}(\mathbf{A})=\int \mathrm{d}^{3} x \operatorname{tr}\left[\mathbf{A}_{2}(x) \partial_{3} \mathbf{A}_{1}(x)-\mathbf{A}_{1}(x) \partial_{3} \mathbf{A}_{2}(x)\right] .
$$


Gauge invariance restricted to gauge functions $\mathbf{g}\left(x_{1}, x_{2}\right)$ should be maintained. Assuming some boundary conditions at Euclidean times $t_{i}$ and $t_{f}$ and integrating over time between $t_{i}$ and $t_{f}$, one finds

$$
\int \mathrm{d}^{2} x \operatorname{tr}\left[\partial_{2} \mathbf{g} \mathbf{g}^{-1}\left(\mathbf{A}_{1}\left(t_{f}\right)-\mathbf{A}_{1}\left(t_{i}\right)\right)-\partial_{1} \mathbf{g} \mathbf{g}^{-1}\left(\mathbf{A}_{2}\left(t_{f}\right)-\mathbf{A}_{2}\left(t_{i}\right)\right)\right]=0,
$$

where $\mathrm{d}^{2} x \equiv \mathrm{d} x_{1} \mathrm{~d} x_{2}$. This condition is automatically satisfied for the quantum partition function at finite temperature. Here, we work at zero temperature and set topological issues aside.

The gauge propagator. For convenience we now set,

$$
\frac{g}{4 \pi}=\frac{N}{\kappa} .
$$

The topological action then reads

$$
\mathcal{S}_{\mathrm{CS}}(\mathbf{A})=\frac{N}{i g} \mathrm{CS}_{3}(\mathbf{A})=\frac{N}{i g} \int \mathrm{d}^{3} x \operatorname{tr}\left[\mathbf{A}_{2}(x) \partial_{3} \mathbf{A}_{1}(x)-\mathbf{A}_{1}(x) \partial_{3} \mathbf{A}_{2}(x)\right] .
$$

The propagator can be calculated by adding external sources. Then,

$$
\mathcal{S}_{\mathrm{CS}}(\mathbf{A}, \mathbf{J})=\mathcal{S}_{\mathrm{CS}}(\mathbf{A})-\int \mathrm{d}^{3} x \operatorname{tr}\left[\mathbf{J}_{1}(x) \mathbf{A}_{1}(x)+\mathbf{J}_{2}(x) \mathbf{A}_{2}(x)\right] .
$$

The solutions of the corresponding equations of motion are

$$
\mathbf{A}_{1}(x)=\frac{i g}{2 N} \int^{x_{3}} \mathrm{~d} x_{3}^{\prime} \mathbf{J}_{2}\left(x_{1}, x_{2}, x_{3}^{\prime}\right), \quad \mathbf{A}_{2}(x)=-\frac{i g}{2 N} \int^{x_{3}} \mathrm{~d} x_{3}^{\prime} \mathbf{J}_{1}\left(x_{1}, x_{2}, x_{3}^{\prime}\right) .
$$

Note that, in the absence of explicit boundary conditions, the inverse of $\partial_{3}$ is only defined up to the addition of a function of $x_{1}, x_{2}$. Independence of boundary terms implies

$$
\int \mathrm{d} x_{3} \mathbf{J}_{1,2}(x)=0 \text {. }
$$

These conditions enforce the remaining gauge invariance of the action corresponding to group elements $\mathbf{g}\left(x_{1}, x_{2}\right)$. They can only be used if the time Fourier components are quantized, for example, in the case of the partition function where fermions satisfy antiperiodic boundary conditions.

The action calculated for the solution then is

$$
\mathcal{S}_{\mathrm{CS}}=\frac{i g}{4 N} \int \mathrm{d}^{3} x \mathrm{~d}^{3} x^{\prime} \delta^{(2)}\left(x-x^{\prime}\right) \operatorname{sgn}\left(x_{3}-x_{3}^{\prime}\right) \operatorname{tr} \mathbf{J}_{2}(x) \mathbf{J}_{1}\left(x^{\prime}\right) .
$$

Using

$$
\operatorname{sgn}(t) \underset{\varepsilon \rightarrow 0}{=} \frac{1}{2 \pi} \int \mathrm{d} \omega \mathrm{e}^{i \omega t} \frac{-2 i \omega}{\omega^{2}+\varepsilon^{2}} \Leftrightarrow-\frac{2 \omega i}{\omega^{2}+\varepsilon^{2}} \underset{\varepsilon \rightarrow 0}{=} \int \mathrm{d} t \mathrm{e}^{-i \omega t} \operatorname{sgn}(t),
$$

we find that the $\mathbf{A}_{1} \mathbf{A}_{2}$ propagator, which is proportional to $(i g / 4 N) \operatorname{sgn}\left(t-t^{\prime}\right) \delta^{(2)}\left(x-x^{\prime}\right)$, has the Fourier representation

$$
\Delta_{\mathbf{A}}(p)=\frac{g}{2 N} \lim _{\varepsilon \rightarrow 0} \frac{p_{3}}{p_{3}^{2}+\varepsilon^{2}}=\frac{g}{2 N} \mathrm{PP} \frac{1}{p_{3}} .
$$




\section{Chern-Simons gauge fields coupled to $\mathrm{U}(N)$ fermions}

We first describe a $\mathrm{U}(N)$ symmetric fermion theory with Chern-Simons term and then study its large $N$ limit.

\subsection{Conventions}

In this section, we assume that $\mathbf{A}_{\mu}$ are antihermitian matrices belonging to the adjoint representation of the group $\mathrm{U}(N)$. As a basis of the Lie algebra, one can take $N^{2}$ antihermitian matrices $\mathbf{t}^{a}$ orthogonal by the trace

$$
\operatorname{trt}^{a} \mathbf{t}^{b}=-\delta_{a b}
$$

With this convention,

$$
\left[\mathbf{t}^{a}, \mathbf{t}^{b}\right]=f_{a b c} \mathbf{t}^{c},
$$

where the structure constants $f_{a b c}$ are real and totally antisymmetric. Moreover, the orthogonality relations (3.1) imply

$$
t_{i j}^{a} t_{\ell k}^{a}=-\delta_{i k} \delta_{j \ell} \Rightarrow \mathbf{t}^{a} \mathbf{t}^{a}=-N \mathbf{1} .
$$

In terms of the $\mathbf{t}^{a}$ matrices, the gauge field then can be parametrized as

$$
\mathbf{A}_{\mu}(x)=A_{\mu}^{a}(x) \mathbf{t}^{a} .
$$

The Chern-Simons action then reads

$$
\mathcal{S}(\mathbf{A})=\frac{i N}{g} \int \mathrm{d}^{3} x\left[A_{2}^{a}(x) \partial_{3} A_{1}^{a}(x)-A_{1}^{a}(x) \partial_{3} A_{2}^{a}(x)\right] .
$$

If we add to the action the source terms

$$
-\int \mathrm{d}^{3} x\left[J_{1}^{a}(x) A_{1}^{a}(x)+J_{2}^{a}(x) A_{2}^{a}(x)\right]
$$

and integrate over the gauge field, we obtain

$$
\mathcal{S}=\frac{g}{4 i N} \int \mathrm{d}^{3} x \mathrm{~d}^{3} x^{\prime} \delta^{(2)}\left(x-x^{\prime}\right) \operatorname{sgn}\left(x_{3}-x_{3}^{\prime}\right) J_{1}^{a}(x) J_{2}^{a}\left(x^{\prime}\right) .
$$

The non-vanishing component of the gauge propagator then is

$$
\Delta_{12}^{a b}\left(x-x^{\prime}\right) \equiv\left\langle A_{1}^{a}(x) A_{2}^{b}\left(x^{\prime}\right)\right\rangle=\frac{i g}{4 N} \delta_{a b} \delta^{(2)}\left(x-x^{\prime}\right) \operatorname{sgn}\left(x_{3}-x_{3}^{\prime}\right) .
$$

In the Fourier representation,

$$
A_{\mu}^{a}(x)=\int \mathrm{d}^{3} p \mathrm{e}^{i p x} \tilde{A}_{\mu}^{a}(p), \quad J_{\mu}^{a}(x)=\int \mathrm{d}^{3} p \mathrm{e}^{i p x} \tilde{J}_{\mu}^{a}(p),
$$

the CS action takes the form

$$
\mathcal{S}(\mathbf{A})=-\frac{2 N}{g}(2 \pi)^{3} \int \mathrm{d}^{3} p \tilde{A}_{1}^{a}(p) p_{3} \tilde{A}_{2}^{a}(-p)
$$


and the source terms become

$$
-(2 \pi)^{3} \int \mathrm{d}^{3} p\left[\tilde{J}_{1}^{a}(-p) \tilde{A}_{1}(p)+\tilde{J}_{2}^{a}(-p) \tilde{A}_{2}(p)\right] .
$$

An integration over the gauge field yields the free energy

$$
\ln \mathcal{Z}=(2 \pi)^{3} \frac{g}{2 N} \int \mathrm{d}^{3} p \tilde{J}_{2}^{a}(-p) \frac{1}{p_{3}} \tilde{J}_{1}^{a}(p) .
$$

The Fourier representation of the gauge field propagator is

$$
\Delta_{\alpha \beta}^{a b}(x)=\frac{1}{(2 \pi)^{3}} \int \mathrm{d}^{3} p \mathrm{e}^{i p x} \tilde{\Delta}_{\alpha \beta}^{a b}(p)
$$

where $\alpha, \beta=\{1,2\}$ with

$$
\tilde{\Delta}_{\alpha \beta}^{a b}(p)=\epsilon_{\alpha \beta} \delta^{a b} \frac{g}{2 N} \frac{1}{p_{3}} .
$$

where $\epsilon_{12}=1=-\epsilon_{21}$. Later we use a simple pole notation, but the pole term always stands for a principal part: $\mathrm{PP}\left(1 / p_{3}\right)$ as depicted in eqs. (2.19), (2.20).

\subsection{The CS action coupled to a gauge-invariant fermion action}

We now add to the Chern-Simons action, quantized in the $\mathbf{A}_{3}=0$ gauge, a $\mathrm{U}(N)$ gaugeinvariant action for an $N$-component spinor field $\psi$,

$$
\mathcal{S}(\psi, \bar{\psi}, \mathbf{A})=\mathcal{S}_{\mathrm{CS}}(\mathbf{A})+\mathcal{S}_{\mathrm{F}}(\psi, \bar{\psi}, \mathbf{A})
$$

with

$$
\mathcal{S}_{\mathrm{F}}(\psi, \bar{\psi}, \mathbf{A})=-\int \mathrm{d}^{3} x \bar{\psi}(x)\left(\not D+M_{0}\right) \psi(x),
$$

where $\boldsymbol{D}$ is defined in (2.2) and we have added a mass term, which violates parity, like the CS action. The $\gamma$-matrices here reduce to the three Pauli matrices:

$$
\sigma_{1}=\left(\begin{array}{ll}
0 & 1 \\
1 & 0
\end{array}\right), \sigma_{2}=\left(\begin{array}{cc}
0 & -i \\
i & 0
\end{array}\right), \sigma_{3}=\left(\begin{array}{cc}
1 & 0 \\
0 & -1
\end{array}\right) .
$$

Regularization. Power counting shows that the coupling constant is dimensionless and, thus, UV divergences are expected. Moreover, the gauge field propagator is not isotropic, which leads to additional difficulties. In a first step, we write formal expressions and postpone the regularization problem, a non-trivial issue in a non-Abelian gauge theory with a Chern-Simons term. Fortunately, we will discover that here the number of independent divergent contributions is small and we will thus deal with the problem in a rather empirical way. 


\subsection{Large $N$ expansion: field integral formalism}

Solving non-Abelian gauge theories in the large $N$ limit is in general a highly non-trivial problem [26], but the problem drastically simplifies when the gauge action reduces to a Chern-Simons term, at least in some specific gauges.

To solve the field theory with the action (3.7) in the large $N$ limit we use a field integral formalism, a standard method to generate large $N$ expansions [17], even if here the context is different and new features can be expected [11].

We thus consider the field integral

$$
\mathcal{Z}=\int[\mathrm{d} \psi][\mathrm{d} \bar{\psi}][\mathrm{d} \mathbf{A}] \mathrm{e}^{-\mathcal{S}(\bar{\psi}, \psi, \mathbf{A})},
$$

where $\mathcal{S}$ is the Euclidean action (3.7).

In the $\mathbf{A}_{3}=0$ gauge, the gauge action is quadratic and the integration over the gauge field can be performed explicitly. One then obtains an effective quartic interaction for the fermions, non-local in Euclidean time. The components of the currents coupled to the gauge field are

$$
J_{\mu}^{a}(t, x)=\bar{\psi}_{\alpha}^{i}(x)\left[\gamma_{\mu}\right]_{\alpha \beta} t_{i j}^{a} \psi_{\beta}^{j}(x),
$$

where the lower index is the spinor index and the upper index the $\mathrm{U}(N)$ vector index.

Using the result (3.4), one finds

$$
\begin{aligned}
\mathcal{S}= & -\int \mathrm{d}^{3} x \bar{\psi}(x)\left(\not \partial+M_{0}\right) \cdot \psi(x)+\frac{g}{4 i N} \int \mathrm{d}^{3} x \mathrm{~d}^{3} x^{\prime} \operatorname{sgn}\left(x_{3}-x_{3}^{\prime}\right) \delta^{(2)}\left(x-x^{\prime}\right) \\
& \times \bar{\psi}_{\alpha}^{i}(x)\left[\sigma_{1}\right]_{\alpha \beta} t_{i j}^{a} \psi_{\beta}^{j}(x) \bar{\psi}_{\gamma}^{k}\left(x^{\prime}\right)\left[\sigma_{2}\right]_{\gamma \delta} t_{k l}^{a} \psi_{\delta}^{l}\left(x^{\prime}\right) .
\end{aligned}
$$

With the help of the identity (3.2), the sum over the group index $a$ can be performed and yields

$$
\begin{aligned}
\mathcal{S} & =-\int \mathrm{d}^{3} x \bar{\psi}(x) \cdot\left(\not \partial+M_{0}\right) \psi(x) \\
& +\frac{g}{2 N} \int \mathrm{d}^{3} x \mathrm{~d}^{3} x^{\prime} \operatorname{sgn}\left(x_{3}-x_{3}^{\prime}\right) \delta_{\mathrm{T}}^{(2)}\left(x-x^{\prime}\right) \bar{\psi}_{1}(x) \cdot \psi_{1}\left(x^{\prime}\right) \bar{\psi}_{2}\left(x^{\prime}\right) \cdot \psi_{2}(x),
\end{aligned}
$$

where

$$
\delta_{\mathrm{T}}^{(2)}\left(x-x^{\prime}\right) \equiv \delta\left(x_{1}-x_{1}^{\prime}\right) \delta\left(x_{2}-x_{2}^{\prime}\right) .
$$

In terms of the Fourier components

$$
\psi(x)=\int \mathrm{d}^{3} p \mathrm{e}^{i p x} \psi(p), \bar{\psi}(x)=\int \mathrm{d}^{3} p \mathrm{e}^{-i p x} \bar{\psi}(p),
$$

the action (3.10) becomes

$$
\begin{aligned}
\mathcal{S}= & -(2 \pi)^{3} \int \mathrm{d}^{3} p \bar{\psi}(p) \cdot\left(i \not p+M_{0}\right) \psi(p) \\
& -\frac{i g}{N}(2 \pi)^{3} \int \mathrm{d}^{3} p \mathrm{~d}^{3} p^{\prime} \mathrm{d}^{3} q \mathrm{~d}^{3} q^{\prime} \delta^{(3)}\left(p+q-p^{\prime}-q^{\prime}\right) \\
& \times \bar{\psi}_{1}(p) \cdot \psi_{1}\left(p^{\prime}\right) \mathrm{PP} \frac{1}{q_{3}-p_{3}^{\prime}} \bar{\psi}_{2}(q) \cdot \psi_{2}\left(q^{\prime}\right) .
\end{aligned}
$$




\subsection{The large $N$ action}

To render the $N$-dependence explicit and be able to study the large $N$ limit, we introduce additional bilocal (in Euclidean time) composite fields $\left\{\rho_{\alpha}\left(t^{\prime}, t, x\right)\right\}$ and $\left\{\lambda_{\alpha}\left(t, t^{\prime}, x\right)\right\}, \alpha=$ 1,2 , with $x \in \mathbb{R}^{2}$, to implement the relation

$$
\rho_{\alpha}\left(t^{\prime}, t, x\right)=\frac{1}{N} \bar{\psi}_{\alpha}(t, x) \cdot \psi_{\alpha}\left(t^{\prime}, x\right) .
$$

Note that only $R_{\alpha}(t, x) \equiv \rho_{\alpha}(t, t, x)$ is gauge invariant.

We then add to the action (3.11),

$$
\mathcal{S}(\lambda, \rho)=\int \mathrm{d}^{2} x \mathrm{~d} t \mathrm{~d} t^{\prime} \sum_{\alpha=1}^{2} \lambda_{\alpha}\left(t, t^{\prime}, x\right)\left[N \rho_{\alpha}\left(t^{\prime}, t, x\right)-\bar{\psi}_{\alpha}(t, x) \cdot \psi_{\alpha}\left(t^{\prime}, x\right)\right] .
$$

This is a generalization (see also ref. [11] and references therein) of the standard method [17] and a reflection of the required gauge field integration. The total action can be written as

$$
\begin{aligned}
\mathcal{S}= & -\int \mathrm{d} t \mathrm{~d}^{2} x \bar{\psi}(t, x) \cdot\left(\not \partial+M_{0}\right) \psi(t, x) \\
& +\frac{1}{2} g N \int \mathrm{d}^{2} x \mathrm{~d} t \mathrm{~d} t^{\prime} \operatorname{sgn}\left(t^{\prime}-t\right) \rho_{1}\left(t^{\prime}, t, x\right) \rho_{2}\left(t, t^{\prime}, x\right) \\
& +\int \mathrm{d}^{2} x \mathrm{~d} t \mathrm{~d} t^{\prime} \sum_{\alpha=1}^{2} \lambda_{\alpha}\left(t, t^{\prime}, x\right)\left[N \rho_{\alpha}\left(t^{\prime}, t, x\right)-\bar{\psi}_{\alpha}(t, x) \cdot \psi_{\alpha}\left(t^{\prime}, x\right)\right] .
\end{aligned}
$$

The integration over $\bar{\psi}$ and $\psi$ can be performed and generates the factor

$$
(\operatorname{det} \mathbf{K})^{N}=\mathrm{e}^{N \operatorname{tr} \ln \mathbf{K}},
$$

where the operator $\mathbf{K}$ is represented by the kernel

$$
K_{\alpha \beta}\left(t, x ; t^{\prime}, x^{\prime}\right)=\left(\not \partial_{\alpha \beta}+\delta_{\alpha \beta} M_{0}\right) \delta\left(t-t^{\prime}\right) \delta^{(2)}\left(x-x^{\prime}\right)+\delta_{\alpha \beta} \lambda_{\alpha}\left(t, t^{\prime}, x\right) \delta^{(2)}\left(x-x^{\prime}\right) .
$$

The large $N$ action then reads

$$
\begin{aligned}
& \mathcal{S}_{N} / N=-\operatorname{tr} \ln \mathbf{K} \\
& +\int \mathrm{d}^{2} x \mathrm{~d} t \mathrm{~d} t^{\prime}\left[\sum_{\alpha=1}^{2} \lambda_{\alpha}\left(t, t^{\prime}, x\right) \rho_{\alpha}\left(t^{\prime}, t, x\right)+\frac{1}{2} g \operatorname{sgn}\left(t^{\prime}-t\right) \rho_{1}\left(t^{\prime}, t, x\right) \rho_{2}\left(t, t^{\prime}, x\right)\right] .
\end{aligned}
$$

The integration over the $\rho$-field is also Gaussian and can be performed. It amounts to replacing $\rho$ by the solution of the field equation.

\subsection{Saddle point equations}

A non-trivial large $N$ limit is obtained by taking the limit with $g$ fixed [26]. The integral is then dominated by saddle points, solution of equations obtained by varying the $\rho$ - and $\lambda$-fields. A variation of the $\rho$-fields yields

$$
\lambda_{1}\left(t, t^{\prime}, x\right)=\frac{1}{2} g \operatorname{sgn}\left(t-t^{\prime}\right) \rho_{2}\left(t, t^{\prime}, x\right),
$$




$$
\lambda_{2}\left(t, t^{\prime}, x\right)=-\frac{1}{2} g \operatorname{sgn}\left(t-t^{\prime}\right) \rho_{1}\left(t, t^{\prime}, x\right) .
$$

Varying the $\lambda$-fields, one obtains

$$
-\rho_{\alpha}\left(t, t^{\prime}, x\right)+\left[\mathbf{K}^{-1}\right]_{\alpha \alpha}\left(t, x ; t^{\prime}, x\right)=0, \alpha=1,2 .
$$

One looks for solutions that do not break time and space translation invariance and, thus,

$$
\lambda_{\alpha}\left(t, t^{\prime} ; x\right)=\lambda_{\alpha}\left(t-t^{\prime}\right), \rho_{\alpha}\left(t, t^{\prime} ; x\right)=\rho_{\alpha}\left(t-t^{\prime}\right) .
$$

Equations (3.16a) and (3.16b) then reduce to

$$
\begin{aligned}
& \lambda_{1}(t)=\frac{1}{2} g \operatorname{sgn}(t) \rho_{2}(t), \\
& \lambda_{2}(t)=-\frac{1}{2} g \operatorname{sgn}(t) \rho_{1}(t) .
\end{aligned}
$$

We define

$$
\tilde{\lambda}_{\alpha}(\omega)=\frac{1}{2 \pi} \int \mathrm{d} t \mathrm{e}^{-i \omega t} \lambda_{\alpha}(t), \tilde{\rho}_{\alpha}(\omega)=\frac{1}{2 \pi} \int \mathrm{d} t \mathrm{e}^{-i \omega t} \rho_{\alpha}(t) .
$$

The operator (3.14) in the Fourier representation takes a diagonal form in $\omega, p$ space $(p \in$ $\mathbb{R}^{2}$ ), with elements the $2 \times 2$ matrices

$$
\tilde{\mathbf{K}}(\omega, p)=i \omega \sigma_{3}+i \not p+M_{0}+\pi\left(\tilde{\lambda}_{1}(\omega)+\tilde{\lambda}_{2}(\omega)\right)+\pi\left(\tilde{\lambda}_{1}(\omega)-\tilde{\lambda}_{2}(\omega)\right) \sigma_{3} .
$$

The action density then reads

$$
\begin{aligned}
\mathcal{S}_{N} / N / \text { volume }= & 2 \pi \int \mathrm{d} \omega \tilde{\rho}_{\alpha}(\omega) \tilde{\lambda}_{\alpha}(\omega)+i g \int \frac{\mathrm{d} \omega \mathrm{d} \omega^{\prime}}{\omega-\omega^{\prime}} \tilde{\rho}_{1}(\omega) \tilde{\rho}_{2}\left(\omega^{\prime}\right) \\
& -\frac{1}{(2 \pi)^{3}} \int \mathrm{d} \omega \mathrm{d}^{2} p \operatorname{tr} \ln \tilde{\mathbf{K}}(\omega, p) .
\end{aligned}
$$

It is convenient to set

$$
\mu_{1}(\omega)=M_{0}+i \omega+2 \pi \tilde{\lambda}_{1}(\omega), \quad \mu_{2}(\omega)=M_{0}-i \omega+2 \pi \tilde{\lambda}_{2}(\omega) .
$$

Then, $\tilde{\mathbf{K}}$ reduces to

$$
\tilde{\mathbf{K}}=i p_{1} \sigma_{1}+i p_{2} \sigma_{2}+\frac{1}{2}\left(\mu_{1}(\omega)+\mu_{2}(\omega)\right)+\frac{1}{2}\left(\mu_{1}(\omega)-\mu_{2}(\omega)\right) \sigma_{3} .
$$

Its inverse can be obtained in the form

$$
\mathcal{D} \tilde{\mathbf{K}}^{-1}=\frac{1}{2}\left(\mu_{1}(\omega)+\mu_{2}(\omega)\right)-\frac{1}{2}\left(\mu_{1}(\omega)-\mu_{2}(\omega)\right) \sigma_{3}-i p_{1} \sigma_{1}-i p_{2} \sigma_{2}
$$

with

$$
\mathcal{D}=p_{1}^{2}+p_{2}^{2}+\mu_{1}(\omega) \mu_{2}(\omega)
$$

Symmetries imply

$$
\tilde{\rho}_{2}(\omega)=\tilde{\rho}_{1}(-\omega)=\rho_{1}^{*}(\omega), \quad \mu_{2}(\omega)=\mu_{1}(-\omega)=\mu_{1}^{*}(\omega) .
$$


After Fourier transformation, eqs. (3.18a) and (3.18b) become

$$
\begin{aligned}
& \mu_{1}(\omega)=M_{0}+i \omega-i g \int \frac{\mathrm{d} \omega^{\prime}}{\omega-\omega^{\prime}} \tilde{\rho}_{2}\left(\omega^{\prime}\right), \\
& \mu_{2}(\omega)=M_{0}-i \omega+i g \int \frac{\mathrm{d} \omega^{\prime}}{\omega-\omega^{\prime}} \tilde{\rho}_{1}\left(\omega^{\prime}\right) .
\end{aligned}
$$

Similarly, eqs. (3.17) become

$$
\begin{aligned}
& \tilde{\rho}_{1}(\omega)=\frac{\mu_{2}(\omega)}{(2 \pi)^{3}} \int \frac{\mathrm{d}^{2} k}{k^{2}+\mu_{1}(\omega) \mu_{2}(\omega)}, \\
& \tilde{\rho}_{2}(\omega)=\frac{\mu_{1}(\omega)}{(2 \pi)^{3}} \int \frac{\mathrm{d}^{2} k}{k^{2}+\mu_{1}(\omega) \mu_{2}(\omega)} .
\end{aligned}
$$

The four equations can be summarized by the unique pair of equations

$$
\begin{aligned}
& \mu_{2}(\omega)=M_{0}-i \omega+i g \int \frac{\mathrm{d} \omega^{\prime}}{\omega-\omega^{\prime}} \tilde{\rho}_{1}\left(\omega^{\prime}\right), \\
& \tilde{\rho}_{1}(\omega)=\frac{\mu_{2}(\omega)}{(2 \pi)^{3}} \int \frac{\mathrm{d}^{2} k}{k^{2}+\left|\mu_{2}(\omega)\right|^{2}} .
\end{aligned}
$$

\subsection{Solution}

It is useful to introduce the function

$$
\Theta(\omega)=\frac{1}{(2 \pi)^{3}} \int \frac{\mathrm{d}^{3} p}{\left(\omega-p_{3}\right)\left(M^{2}+p^{2}\right)} .
$$

Here, and later, $1 /\left(\omega-p_{3}\right)$ stands for $P P\left[1 /\left(\omega-p_{3}\right)\right]$. The function $\Theta$ requires some regularization but, with a suitable space symmetric regularization, has a regularizationindependent limit because the integrand is globally odd: it is most easily calculated in real space (see appendix A.1) and one finds

$$
\Theta(\omega)=\frac{1}{4 \pi} \arctan \left(\frac{\omega}{M}\right) .
$$

Then, initially motivated by perturbative calculations (see section 4.1), we set

$$
\tilde{\rho}_{1}(\omega)=\tilde{\rho}_{2}^{*}(\omega)=(M-i \omega) \exp [i g \Theta(\omega)] \frac{1}{(2 \pi)^{3}} \int \frac{\mathrm{d}^{2} p}{p^{2}+\omega^{2}+M^{2}},
$$

where $M$ is a free mass parameter that will be later identified with the fermion physical mass.

From equation (3.24a), one then infers

$$
\mu_{2}(\omega)=M_{0}-i \omega-\frac{i g}{(2 \pi)^{3}} \int \mathrm{d}^{3} p \frac{\left(M-i p_{3}\right)}{\left(p_{3}-\omega\right)\left(p^{2}+M^{2}\right)} \exp \left[i g \Theta\left(p_{3}\right)\right] .
$$

The integral can be performed by expanding in powers of $g$, symmetrizing over all integration momenta and using the identities (B.2)-(B.3), a technique that will be used systematically in many places in this work. 
Using the identity (B.2), one verifies that, for $n>0$,

$$
-\frac{i}{(2 \pi)^{3}} \int \mathrm{d}^{3} p \frac{\left(M-i p_{3}\right)}{\left(p_{3}-\omega\right)\left(p^{2}+M^{2}\right)} \Theta^{n}\left(p_{3}\right)=\frac{i}{n+1}(M-i \omega) \Theta^{n+1}(\omega) .
$$

For $n=0$, an additional term is generated (identity (B.3)) and one finds

$$
-\frac{i}{(2 \pi)^{3}} \int \mathrm{d}^{3} p \frac{\left(M-i p_{3}\right)}{\left(p_{3}-\omega\right)\left(p^{2}+M^{2}\right)}=-\Omega_{1}(M)+i(M-i \omega) \Theta(\omega),
$$

where we define more generally, for later purpose,

$$
\Omega_{n}(M)=\frac{1}{(2 \pi)^{3}} \int \frac{\mathrm{d}^{3} p}{\left(p^{2}+M^{2}\right)^{n}} .
$$

For $n=1$, it is a divergent quantity that has to be regularized.

One obtains

$$
\mu_{2}(\omega)=M_{0}-M-g \Omega_{1}(M)+(M-i \omega) \exp [i g \Theta(\omega)]
$$

We then choose the mass parameter $M$ to be the solution of the gap equation

$$
M_{0}=M+g \Omega_{1}(M)
$$

and we verify later that $M$ is then the fermion physical mass.

We conclude

$$
\mu_{2}(\omega)=(M-i \omega) \exp [i g \Theta(\omega)]
$$

and, thus,

$$
\left|\mu_{2}(\omega)\right|^{2}=M^{2}+\omega^{2} .
$$

The verification of equation (3.24b) is then straightforward.

The constants $\boldsymbol{\Omega}_{\boldsymbol{n}}(\boldsymbol{M})$. For $n>1, \Omega_{n}(M)$, defined by eq. (3.28) is given by

$$
\Omega_{n}(M)=\frac{1}{8 \pi^{3 / 2}} \frac{\Gamma(n-3 / 2)}{(n-1) !} M^{3-2 n} .
$$

For $n=1$, assuming some regularization, we define the UV cut-off $\Lambda$ by

$$
\Omega_{1}(0)=\int \frac{\mathrm{d}^{3} p}{(2 \pi)^{3}} \frac{1}{p^{2}} \equiv \frac{\Lambda}{4 \pi} .
$$

In dimensional regularization (which we will not use) this contribution vanishes.

Then,

$$
\Omega_{1}(M)=\frac{\Lambda-|M|}{4 \pi}+O\left(M^{2} / \Lambda\right) .
$$

In what follows we will omit everywhere the $M^{2} / \Lambda$ correction. 


\subsection{The free energy density}

We define the free energy density, which is proportional to the ground state energy, dividing by a factor $N$, by

$$
W=\frac{1}{N V} \ln \left(\mathcal{Z} / \mathcal{Z}_{0}\right)
$$

where $\mathcal{Z}$ is the partition function, $\mathcal{Z}_{0}$ a normalization and $V$ the volume. From eq. (3.19), one infers, in the large $N$ limit,

$$
\begin{aligned}
W= & -2 \pi \int \mathrm{d} \omega \tilde{\rho}_{\alpha}(\omega) \tilde{\lambda}_{\alpha}(\omega)-i g \int \frac{\mathrm{d} \omega \mathrm{d} \omega^{\prime}}{\omega-\omega^{\prime}} \tilde{\rho}_{1}(\omega) \tilde{\rho}_{2}\left(\omega^{\prime}\right) \\
& +\frac{1}{(2 \pi)^{3}} \int \mathrm{d} \omega \mathrm{d}^{2} p \operatorname{tr} \ln \tilde{\mathbf{K}}(\omega, p),
\end{aligned}
$$

where $\tilde{\lambda}$ and $\tilde{\rho}$ have to be replaced by the solutions of the saddle point equations

$$
\begin{aligned}
& \tilde{\lambda}_{1}=\frac{1}{2 \pi}(M+i \omega)\left(\mathrm{e}^{-i g \Theta(\omega)}-1\right), \\
& \tilde{\rho}_{1}=(M-i \omega) \mathrm{e}^{i g \Theta(\omega)} \frac{1}{(2 \pi)^{3}} \int \frac{\mathrm{d}^{2} k}{k^{2}+\omega^{2}+M^{2}},
\end{aligned}
$$

and complex conjugate for $\tilde{\lambda}_{2}, \tilde{\rho}_{2}$. Moreover,

$$
\operatorname{tr} \ln \tilde{\mathbf{K}}=\ln \left(p^{2}+\omega^{2}+M^{2}\right) .
$$

Then, from the saddle point equation,

$$
i g \int \mathrm{d} \omega^{\prime} \frac{\tilde{\rho}_{2}\left(\omega^{\prime}\right)}{\omega-\omega^{\prime}}=(M+i \omega)\left(\mathrm{e}^{-i g \Theta(\omega)}-1\right) .
$$

Thus,

$$
\begin{aligned}
W= & \frac{1}{(2 \pi)^{3}} \int \mathrm{d}^{3} p \ln \left(1+M^{2} / p^{2}\right) \\
& +i g \int \frac{\mathrm{d} \omega \mathrm{d} \omega^{\prime}}{\omega-\omega^{\prime}}(M-i \omega)\left(M+i \omega^{\prime}\right) \mathrm{e}^{i g\left[\Theta(\omega)-\Theta\left(\omega^{\prime}\right)\right]} \\
& \times \frac{1}{(2 \pi)^{6}} \int \frac{\mathrm{d}^{2} p \mathrm{~d}^{2} p^{\prime}}{\left(p^{2}+\omega^{2}+M^{2}\right)\left(p^{\prime 2}+\omega^{\prime 2}+M^{2}\right)} .
\end{aligned}
$$

Expanding in powers of $g$, replacing the functions $\Theta$ by their integral representation and evaluating each term using identities of the kind explained in section B.1, one obtains

$$
W=\frac{1}{(2 \pi)^{3}} \int \mathrm{d}^{3} p \ln \left(1+M^{2} / p^{2}\right)+g M \Omega_{1}^{2}(M)+\frac{1}{3} g^{2} \Omega_{1}^{3}(M),
$$

where the first term has still to be regularized. Differentiating the first term, one finds

$$
\frac{\partial}{\partial M} \frac{1}{(2 \pi)^{3}} \int \mathrm{d}^{3} p \ln \left(1+M^{2} / p^{2}\right)=2 M \Omega_{1}(M) .
$$

Using the explicit form (3.34) and integrating back, one obtains

$$
\frac{1}{(2 \pi)^{3}} \int \mathrm{d}^{3} p \ln \left(1+M^{2} / p^{2}\right)=\frac{\Lambda}{4 \pi} M^{2}-\frac{|M|^{3}}{6 \pi}+\text { const. . }
$$

We note that $W$ is even in the change $M \leftrightarrow-M, g \Leftrightarrow-g$ or, equivalently $M_{0} \leftrightarrow-M_{0}$, $g \Leftrightarrow-g$. 


\subsection{The fermion two-point function for $N$ large}

The fermion two-point function has the general form

$$
\left\langle\psi_{\alpha}^{i}(x) \bar{\psi}_{\beta}^{j}\left(x^{\prime}\right)\right\rangle_{0}=\delta_{i j} W_{\alpha \beta}^{(2)}\left(x-x^{\prime}\right)
$$

with

$$
W^{(2)}(x)=\frac{1}{(2 \pi)^{3}} \int \mathrm{d}^{3} p \mathrm{e}^{i p x} \tilde{W}^{(2)}(p) .
$$

In the large $N$ limit, the fermion two-point function is obtained by inverting the operator (3.14) at the saddle point. Using the expression (3.21) and replacing the functions $\mu_{\alpha}$ by the explicit solutions (3.30) of the saddle point equations, one finds

$$
\mathcal{D}(p)=p^{2}+M^{2},
$$

and thus

$$
\tilde{\mathbf{K}}^{-1}=-\frac{i p_{1} \sigma_{1}+i p_{2} \sigma_{2}+\left(i \omega \sigma_{3}-M\right) \exp \left[i g \sigma_{3} \Theta(\omega)\right]}{p^{2}+M^{2}} .
$$

We note that

$$
\tilde{\mathbf{K}}^{-1}=-\exp \left[\frac{i}{2} g \sigma_{3} \Theta(\omega)\right] \frac{(i \not p-M)}{p^{2}+M^{2}} \exp \left[\frac{i}{2} g \sigma_{3} \Theta(\omega)\right] .
$$

It is convenient to define

$$
U(\omega)=\exp \left[\frac{1}{2} i g \sigma_{3} \Theta(\omega)\right] .
$$

The dressed fermion fermion propagator then takes the form

$$
\tilde{W}^{(2)}(p)=-\tilde{\mathbf{K}}^{-1}(p)=U\left(p_{3}\right) \frac{(i \not p-M)}{p^{2}+M^{2}} U\left(p_{3}\right) .
$$

This expression confirms that the parameter $M$ is the physical fermion mass. Moreover, this form suggests that, after mass renormalization and a non-local phase transformation, the fermion theory is equivalent to a free theory.

Correspondingly, the two-point vertex function, inverse of $W^{(2)}$, can be written as

$$
\tilde{\Gamma}^{(2)}(p)=-U^{-1}\left(p_{3}\right)(i \not p+M) U^{-1}\left(p_{3}\right) .
$$

More explicit expressions, using the explicit form of $\Theta$, are

$$
\begin{aligned}
& \tilde{W}^{(2)}(p)=\frac{1}{p^{2}+M^{2}}\left\{i p_{1} \sigma_{1}+i p_{2} \sigma_{2}-\sqrt{M^{2}+p_{3}^{2}} \exp \left[i \sigma_{3}(g-4 \pi) \Theta\left(p_{3}\right)\right]\right\} \\
& \tilde{\Gamma}^{(2)}(p)=i p_{1} \sigma_{1}+i p_{2} \sigma_{2}+\sqrt{M^{2}+p_{3}^{2}} \exp \left[-i \sigma_{3}(g-4 \pi) \Theta\left(p_{3}\right)\right] .
\end{aligned}
$$

Finally,

$$
\int \mathrm{d}^{2} q \tilde{W}^{(2)}(q)=\left(i p_{3} \sigma_{3}-M\right) U^{2}\left(p_{3}\right) \frac{1}{(2 \pi)^{3}} \int \frac{\mathrm{d}^{3} q}{\left(p_{3}-q_{3}\right)\left(M^{2}+q^{2}\right)}
$$

a result consistent with equation (3.27). 


\subsection{Gauge-invariant observables}

To be able to distinguish gauge artefacts from gauge independent properties, it is necessary to calculate gauge invariant observables. The $\psi$-field two-point function is not gauge invariant, except in the limit of coinciding points. Two gauge invariant operators are (no summation assumed)

$$
R_{\alpha}(x)=\frac{1}{N} \bar{\psi}_{\alpha}(x) \cdot \psi_{\alpha}(x)
$$

Below, we consider the scalar combination

$$
R(x)=R_{1}(x)+R_{2}(x)
$$

and the third component of the current

$$
J_{3}(x)=i\left(R_{1}(x)-R_{2}(x)\right) .
$$

Expectation value. We can already determine the expectation value of the operator $R_{\alpha}(x)$. The equal-time expectation value of $\rho_{1}$ is given by

$$
\left\langle R_{1}(x)\right\rangle=\int \mathrm{d} \omega \tilde{\rho}_{1}(\omega)=\frac{1}{(2 \pi)^{3}} \int \mathrm{d}^{3} p \frac{M-i p_{3}}{p^{2}+M^{2}} \exp \left[i g \Theta\left(p_{3}\right)\right] .
$$

Expanding in powers of $g$ and replacing $\Theta\left(p_{3}\right)$ by its integral representation (3.25), symmetrizing the integrand with respect to $p$ and all the other integration momenta, one verifies that all terms vanish except the two first ones. This result, and other similar ones, rely on the identities (B.5). Since the result is real, $\left\langle J_{3}\right\rangle=0$ and

$$
\langle R\rangle=\left\langle R_{1}+R_{2}\right\rangle=2\left\langle\rho_{1}\right\rangle=2 M \Omega_{1}(M)+g \Omega_{1}^{2}(M) .
$$

We find that the expectation value, which is gauge invariant, is indeed given by an explicitly $O(3)$ symmetric expression, in the sense that it makes no reference to the gauge propagator and is expressed in terms of an obviously covariant integral. In terms of the explicit expression (3.34), for $M>0$ it reads

$$
\langle R\rangle=g \frac{\Lambda^{2}}{16 \pi^{2}}+\frac{\Lambda M}{2 \pi}\left(1-\frac{g}{4 \pi}\right)-\frac{M^{2}}{2 \pi}\left(1-\frac{g}{8 \pi}\right) .
$$

$\boldsymbol{R}$ expectation value and ground state energy. The normalized free energy density defined by eq. (3.35) and calculated in section 3.7, is given by (eq. (3.36))

$$
W=\frac{1}{(2 \pi)^{3}} \int \mathrm{d}^{3} p \ln \left(1+M^{2} / p^{2}\right)+g M \Omega_{1}^{2}(M)+\frac{1}{3} g^{2} \Omega_{1}^{3}(M) .
$$

Then,

$$
\langle R\rangle=\frac{\partial W}{\partial M_{0}},
$$

which can be rewritten as (eq. (3.29))

$$
\frac{\partial W}{\partial M}=\frac{\partial W}{\partial M_{0}} \frac{\partial M_{0}}{\partial M}=\langle R\rangle\left(1-2 g M \Omega_{2}(M)\right)=\langle R\rangle\left(1-\frac{g}{4 \pi}\right) .
$$

One then verifies the consistency between the expressions (3.47) and (3.46). 

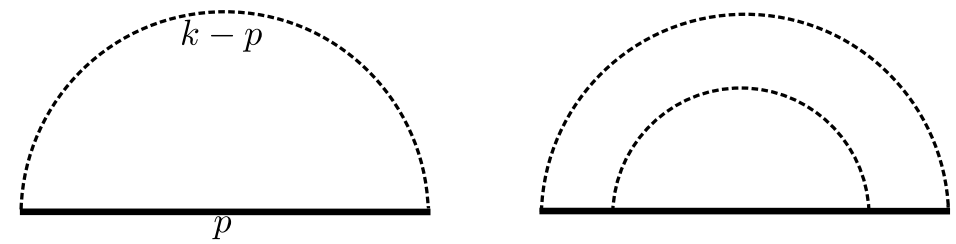

Figure 1. One- and two-loop contributions to the two-point vertex function. Dotted lines represent gauge propagators.

Connected $\boldsymbol{R}$ correlation function at zero momentum. The $R$ two-point function, at zero momentum is obtained by differentiating $\langle R\rangle$ with respect to $M_{0}$. One then finds (the subscript $c$ stands for connected)

$$
\begin{aligned}
\langle\tilde{R}(0) \tilde{R}(0)\rangle_{\mathrm{c}} & =\frac{\partial\langle R\rangle}{\partial M_{0}}=\frac{\partial\langle R\rangle}{\partial M} / \frac{\partial M_{0}}{\partial M} \\
& =2 \Omega_{1}(M)-\frac{4 M^{2} \Omega_{2}(M)}{1-2 g M \Omega_{2}(M)}=\frac{\Lambda}{2 \pi}-\frac{M}{\pi} \frac{(1-g / 8 \pi)}{(1-g / 4 \pi)} .
\end{aligned}
$$

This quantity requires only an additive renormalization: $\langle\tilde{R}(0) \tilde{R}(0)\rangle_{\mathrm{c}}-\Lambda / 2 \pi$ is finite.

Higher order functions can be obtained by further differentiation. here differentiating again, one obtains (still $M>0$ )

$$
\langle\tilde{R}(0) \tilde{R}(0) \tilde{R}(0)\rangle_{\mathrm{c}}=-\frac{1}{\pi} \frac{(1-g / 8 \pi)}{(1-g / 4 \pi)^{2}},
$$

which is finite. All other connected correlation functions then vanish for $\Lambda \rightarrow \infty$.

\section{Perturbative calculations at large $N$}

It is interesting to see how some results that we have obtained by field integral techniques emerge from perturbative calculations. Therefore, in this section, we calculate a few orders of some of the quantities that we have determined in the preceding sections.

\subsection{The fermion two-point function at two-loop order}

We first expand the fermion two-point function, which is not a gauge-invariant quantity but which has been determined exactly in the large $N$ limit (equation (3.39)).

The fermion propagator. The bare fermion propagator $\left(\langle\bullet\rangle_{0}\right.$ means Gaussian expectation value) is given by

$$
\left\langle\psi_{\alpha}^{i}(x) \bar{\psi}_{\beta}^{j}\left(x^{\prime}\right)\right\rangle_{0}=\delta_{i j} \int \frac{\mathrm{d}^{3} p}{(2 \pi)^{3}} \mathrm{e}^{i p\left(x-x^{\prime}\right)}\left[\tilde{\Delta}_{\mathrm{F}}\right]_{\alpha \beta}(p)
$$

with

$$
\tilde{\boldsymbol{\Delta}}_{\mathrm{F}}(p)=\frac{i \not p-M_{0}}{p^{2}+M_{0}^{2}}
$$




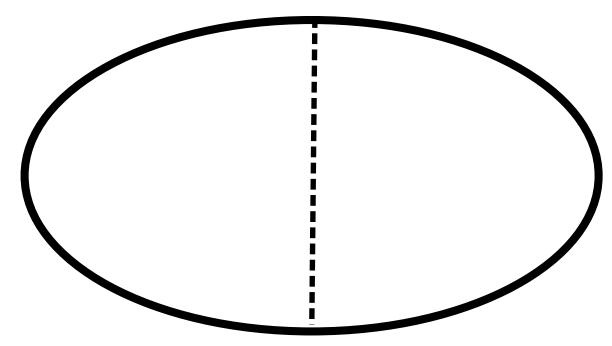

Figure 2. Two-loop contribution to the vacuum energy. Dotted lines represent gauge propagators.

One-loop calculation: the two-point vertex function. Perturbative calculations of the two-point vertex function or mass operator involve the one-loop diagram of figure 1. It can be written as

$$
\Sigma_{1}(k)=\frac{1}{2} \int \frac{\mathrm{d}^{3} p}{(2 \pi)^{3}} \frac{\left[\sigma_{1}\left(i \not p-M_{0}\right) \sigma_{2}-\sigma_{2}\left(i \not p-M_{0}\right) \sigma_{1}\right]}{\left(k_{3}-p_{3}\right)\left(p^{2}+M_{0}^{2}\right)} .
$$

The evaluation is simple and yields

$$
\Sigma_{1}(k)=-\Omega_{1}\left(M_{0}\right)+\left(k_{3}-i M_{0} \sigma_{3}\right) \Theta_{0}\left(k_{3}\right),
$$

where $\Theta_{0}$ is the function (3.25) in which $M$ is replaced by $M_{0}$. The expression in the case of $M_{0}=0$ agrees with the calculation in the temporal gauge in ref. [11] at one-loop order.

The two-point vertex function is then

$$
\begin{aligned}
\tilde{\Gamma}^{(2)}(k) & =-M_{0}-i \not k-g \Sigma_{1}(k)+O\left(g^{2}\right) \\
& =-M_{0}-i \not k+g \Omega_{1}\left(M_{0}\right)-g\left(k_{3}-i M_{0} \sigma_{3}\right) \Theta_{0}\left(k_{3}\right)+O\left(g^{2}\right) .
\end{aligned}
$$

After introduction of the physical fermion mass (3.29), which amounts to a mass renormalization, the expression agrees with the expansion of the result (3.40) at order $g$.

Two-loop order. After mass renormalization (equation (3.29)), the two-loop contribution $\Sigma_{2}$ to the two-point vertex function (two-loop diagram of figure 1) is proportional to

$$
\Sigma_{2} \propto-\frac{1}{(2 \pi)^{3}} \int \frac{\mathrm{d}^{3} q\left(i q_{3} \sigma_{3}+M\right)}{\left(q^{2}+M^{2}\right)\left(k_{3}-q_{3}\right)} \Theta\left(q_{3}\right)=-\frac{1}{2}\left(M+i \sigma_{3} k_{3}\right) \Theta^{2}\left(k_{3}\right),
$$

which is again consistent with the expansion of the expression (3.40).

\subsection{The ground state (or vacuum) energy up to three loops}

We have defined the normalized, gauge-independent, free energy density by equation (3.35). We expand it as

$$
W=\frac{1}{N V} \ln \left(\mathcal{Z} / \mathcal{Z}_{0}\right)=W_{0}+g W_{1}+g^{2} W_{2}
$$

and keep only the leading terms for $N$ large. Note that in view of the remark after eq. (3.45), the expectation value $\langle R\rangle$ at leading $N$ contains only terms up to order $g^{2}$ and thus in view 


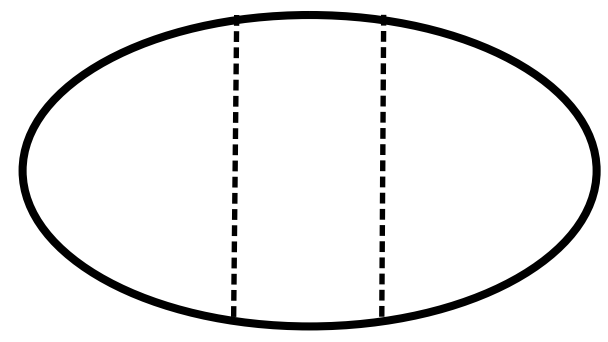

Figure 3. Three-loop contribution to the vacuum energy. Dotted lines represent gauge propagators.

of eq. (3.47) there is no term of order $O\left(g^{3}\right)$ in eq. (4.4). This observation was also made in ref. [11] where the free energy has been calculated in the light-cone gauge.

We choose $\mathcal{Z}_{0}$ such that

$$
W_{0}=\operatorname{tr} \ln \left(1+\not \partial / M_{0}\right)=\frac{1}{(2 \pi)^{3}} \int \mathrm{d}^{3} p \ln \left(1+M_{0}^{2} / p^{2}\right) .
$$

The two-loop contribution is (figure 2)

$$
W_{1}=-\frac{1}{4} \int \frac{\mathrm{d}^{3} p \mathrm{~d}^{3} q}{(2 \pi)^{6}\left(q_{3}-p_{3}\right)} \operatorname{tr}\left[\tilde{\Delta}_{\mathrm{F}}(q)\left(\sigma_{1} \tilde{\Delta}_{\mathrm{F}}(p) \sigma_{2}-\sigma_{2} \tilde{\Delta}_{\mathrm{F}}(p) \sigma_{1}\right)\right] .
$$

Calculating the trace, one verifies that the gauge propagator cancels and one finds

$$
W_{1}=-M_{0} \Omega_{1}^{2}\left(M_{0}\right) .
$$

Three-loop order. The three-loop order calculation (order $g^{2}$, figure 3) is lengthier. Setting

one obtains

$$
S_{2}=\Delta_{\mathrm{F}}(q)\left(\sigma_{1} \tilde{\Delta}_{\mathrm{F}}(p) \sigma_{2}-\sigma_{2} \tilde{\Delta}_{\mathrm{F}}(p) \sigma_{1}\right) \tilde{\Delta}_{\mathrm{F}}(q)
$$

$$
W_{2}=-\frac{1}{8} \int \frac{\mathrm{d}^{3} p \mathrm{~d}^{3} q \mathrm{~d}^{3} r}{(2 \pi)^{9}\left(r_{3}-q_{3}\right)\left(q_{3}-p_{3}\right)} \operatorname{tr}\left\{\tilde{\Delta}_{\mathrm{F}}(r)\left[\sigma_{1} S_{2}(p, q) \sigma_{2}-\sigma_{2} S_{2}(p, q) \sigma_{1}\right]\right\} .
$$

After evaluating the trace, the expression decomposes into the sum of two terms,

$$
\begin{aligned}
W_{2,1} & =-\int \frac{\mathrm{d}^{3} p \mathrm{~d}^{3} q \mathrm{~d}^{3} r}{(2 \pi)^{9}\left(r_{3}-q_{3}\right)\left(q_{3}-p_{3}\right)} \frac{M_{0}^{2}+p_{3} r_{3}}{\left(p^{2}+M_{0}^{2}\right)\left(q^{2}+M_{0}^{2}\right)\left(r^{2}+M_{0}^{2}\right)} \\
W_{2,2} & =-\int \frac{\mathrm{d}^{3} p \mathrm{~d}^{3} q \mathrm{~d}^{3} r}{(2 \pi)^{9}} \frac{2 M_{0}^{2}}{\left(p^{2}+M_{0}^{2}\right)\left(q^{2}+M_{0}^{2}\right)^{2}\left(r^{2}+M_{0}^{2}\right)} .
\end{aligned}
$$

The first contribution can now symmetrized over $r_{3}, p_{3}, q_{3}$. The gauge field poles then cancel and the result is

$$
W_{2}=-2 M_{0}^{2} \Omega_{1}^{2}\left(M_{0}\right) \Omega_{2}\left(M_{0}\right)+\frac{1}{3} \Omega_{1}^{3}\left(M_{0}\right) .
$$

The expansion of the free energy expressed in terms of the fermion physical mass (3.29) then becomes

$$
W=\int \frac{\mathrm{d}^{3} p}{(2 \pi)^{3}} \ln \left(1+M^{2} / p^{2}\right)+M \Omega_{1}^{2}(M) g+\frac{1}{3} \Omega_{1}^{3}(M) g^{2},
$$

in agreement with the expression (3.47). 


\section{Correlation functions involving $R=\bar{\psi} \cdot \psi$ at large $N$}

We now determine the $\langle(\bar{\psi} \cdot \psi) \psi \bar{\psi}\rangle$ vertex function at leading order for $N$ large. But before a simple remark is useful.

\subsection{A simple transformation}

To generate insertions of $R_{\alpha}$ operators, we introduce non-diagonal, space-dependent, mass terms. A simple limit corresponds to correlations of $\int \mathrm{d}^{2} x R_{\alpha}\left(x_{3}, x\right)$. They can be generated by

$$
\mathcal{S}_{M}=\int \mathrm{d}^{3} x \sum_{\alpha} \bar{\psi}_{\alpha}(x) M_{\alpha}\left(x_{3}\right) \cdot \psi_{\alpha}(x)
$$

We then make on $\psi$ a phase transformation of the form

$$
\psi(x) \mapsto \mathrm{e}^{\varphi\left(x_{3}\right)} \psi(x), \quad \bar{\psi}(x) \mapsto \mathrm{e}^{-\varphi\left(x_{3}\right)} \bar{\psi}(x) .
$$

In the action (3.8) (where $M$ is replaced by $M_{\alpha}\left(x_{3}\right)$ ), only the $x_{3}$ derivative is modified:

$$
\mathcal{S}_{\mathrm{F}} \mapsto \mathcal{S}_{\mathrm{F}}-\int \mathrm{d}^{3} x \bar{\psi}(x) \sigma_{3} \partial_{3} \varphi\left(x_{3}\right) \cdot \psi(x)
$$

Expressing the diagonal matrix $\mathbf{M}$ of eigenvalues $M_{1}, M_{2}$ in the form

$$
\mathbf{M}=\frac{1}{2}\left(M_{1}+M_{2}\right) \mathbf{1}+\frac{1}{2}\left(M_{1}-M_{2}\right) \sigma_{3}
$$

and choosing $\varphi\left(x_{3}\right)$ such that

$$
\partial_{3} \varphi\left(x_{3}\right)=\frac{1}{2}\left(M_{1}\left(x_{3}\right)-M_{2}\left(x_{3}\right)\right),
$$

we reduce the mass term (5.1) to

$$
\mathcal{S}_{M}=\frac{1}{2} \int \mathrm{d}^{3} x\left(M_{1}\left(x_{3}\right)+M_{2}\left(x_{3}\right)\right) \bar{\psi}_{\alpha}(x) \cdot \psi_{\alpha}(x) .
$$

We conclude that the expectation values of products of $\int \mathrm{d}^{2} x R_{\alpha}\left(x_{3}, x\right)$ operators are trivially related to the expectation values of $\int \mathrm{d}^{2} x R\left(x_{3}, x\right)$ and this provides some checks in the calculations.

\subsection{Field integral formalism: expansion at the saddle point}

Connected correlation functions involving the $R$ operator at non-vanishing momenta can be calculated for $N$ large by using the field integral formulation of section 3.3. The calculation involves expanding the large $N$ action (3.15) at the saddle point to quadratic order and performing a Gaussian integration. The quadratic form depends on the second functional derivatives of the large $N$ action with respect to $\rho, \lambda$ at the saddle point. The less trivial part is the second functional derivative of the determinant with respect to $\lambda$. 
The second functional derivative of $\operatorname{tr} \ln \mathbf{K}$. Calculating the second functional derivative of the trace of the logarithm of the operator (3.14) with respect to $\left\{\lambda_{\alpha}\left(t_{1}, t_{2}, x\right), \lambda_{\beta}\left(t_{1}^{\prime}, t_{2}^{\prime}, x^{\prime}\right)\right\}$, at the saddle point, we obtain the formal expression (no summation on $\alpha, \beta$ implied)

$$
-\left[\mathbf{K}^{-1}\right]_{\alpha \beta}\left(t_{2}, x ; t_{1}^{\prime}, x^{\prime}\right)\left[\mathbf{K}^{-1}\right]_{\beta \alpha}\left(t_{2}^{\prime}, x^{\prime} ; t_{1}, x\right) .
$$

Other functional derivatives. The second functional derivative of the large $N$ action (3.15) with respect to $\left\{\lambda_{\alpha}\left(t_{1}, t_{2}, x\right), \rho_{\beta}\left(t_{1}^{\prime}, t_{2}^{\prime}, x^{\prime}\right)\right\}$ is

$$
\delta\left(t_{1}^{\prime}-t_{2}\right) \delta\left(t_{2}^{\prime}-t_{1}\right) \delta^{(2)}\left(x-x^{\prime}\right) \delta_{\alpha \beta}
$$

and, finally, with respect to $\left\{\rho_{\alpha}\left(t_{1}, t_{2}, x\right), \rho_{\beta}\left(t_{1}^{\prime}, t_{2}^{\prime}, x^{\prime}\right)\right\}$,

$$
\frac{1}{2} g \operatorname{sgn}\left(t_{1}-t_{2}\right) \delta\left(t_{1}^{\prime}-t_{2}\right) \delta\left(t_{2}^{\prime}-t_{1}\right) \delta^{(2)}\left(x-x^{\prime}\right)\left(\delta_{\alpha 1} \delta_{\beta 2}-\delta_{\alpha 2} \delta_{\beta 1}\right) .
$$

This determines the $2 \times 2$ matrix of functional derivatives, which has still to be inverted and this is the non-trivial part of the calculation of $R$ correlation functions.

Fourier representation. We introduce the Fourier representation

$$
\left[\mathbf{K}^{-1}\right]_{\alpha \beta}\left(t, x ; t^{\prime}, x^{\prime}\right)=\frac{1}{(2 \pi)^{3}} \int \mathrm{d}^{2} p \mathrm{~d} \omega \mathrm{e}^{i \omega\left(t-t^{\prime}\right)+i p\left(x-x^{\prime}\right)}\left[\tilde{\mathbf{K}}^{-1}\right]_{\alpha \beta}(\omega, p),
$$

where the explicit expression is given by eq. (3.37). Thus,

$$
\begin{aligned}
& {\left[\mathbf{K}^{-1}\right]_{\alpha \beta}\left(t_{2}, x ; t_{1}^{\prime}, x^{\prime}\right)\left[\mathbf{K}^{-1}\right]_{\beta \alpha}\left(t_{2}^{\prime}, x^{\prime} ; t_{1}, x\right)} \\
& =\frac{1}{(2 \pi)^{6}} \int \mathrm{d}^{2} p \mathrm{~d}^{2} p^{\prime} \mathrm{d} \omega \mathrm{d} \omega^{\prime} \mathrm{e}^{i \omega\left(t_{2}-t_{1}^{\prime}\right)+i \omega^{\prime}\left(t_{2}^{\prime}-t_{1}\right)+i\left(p-p^{\prime}\right)\left(x-x^{\prime}\right)} \\
& \quad \times\left[\tilde{\mathbf{K}}^{-1}\right]_{\alpha \beta}(\omega, p)\left[\tilde{\mathbf{K}}^{-1}\right]_{\beta \alpha}\left(\omega^{\prime}, p^{\prime}\right) .
\end{aligned}
$$

In a global Fourier representation, this yields

$$
\frac{1}{(2 \pi)^{3}} \delta^{(2)}\left(p+p^{\prime}\right) \delta\left(\omega_{1}+\omega_{2}^{\prime}\right) \delta\left(\omega_{2}+\omega_{1}^{\prime}\right) \int \mathrm{d}^{2} q\left[\tilde{\mathbf{K}}^{-1}\right]_{\alpha \beta}\left(\omega_{2}, q\right)\left[\tilde{\mathbf{K}}^{-1}\right]_{\beta \alpha}\left(\omega_{2}^{\prime}, p-q\right) .
$$

We set

$$
\mathbf{G}_{\alpha \beta}\left(\omega, \omega^{\prime}, p\right)=\frac{1}{(2 \pi)^{3}} \int \mathrm{d}^{2} q\left[\tilde{\mathbf{K}}^{-1}\right]_{\alpha \beta}(\omega, q)\left[\tilde{\mathbf{K}}^{-1}\right]_{\beta \alpha}\left(\omega^{\prime}, p-q\right)=\mathbf{G}_{\beta \alpha}\left(\omega, \omega^{\prime}, p\right) .
$$

In the same way, the $\lambda \rho$ element becomes

$$
\delta\left(\omega_{1}+\omega_{2}^{\prime}\right) \delta\left(\omega_{2}+\omega_{1}^{\prime}\right) \delta^{(2)}\left(p+p^{\prime}\right) \delta_{\alpha \beta}
$$

and the $\rho \rho$ element

$$
\frac{1}{2} i g\left(\delta_{\alpha 1} \delta_{\beta 2}-\delta_{\alpha 2} \delta_{\beta 1}\right) \delta^{(2)}\left(p+p^{\prime}\right) \delta\left(\omega_{1}+\omega_{2}+\omega_{1}^{\prime}+\omega_{2}^{\prime}\right) \frac{1}{\omega_{1}+\omega_{2}^{\prime}} .
$$

We note that all expressions are diagonal in the momentum variables. 
Remarks. The calculation of the inverse operator involves solving two coupled integral equations, a problem we discuss in the coming sections. Note that if the inverse operator is expanded in powers of the coupling $g$, it generates a sum of ladder diagrams with the dressed fermion propagator (3.39), which sums all propagator corrections.

To calculate correlation functions of $R_{\alpha}(t, x)=\bar{\psi}_{\alpha}(t, x) \cdot \psi_{\alpha}(t, x) / N$, one needs the operator sources for the vectors $\rho_{\alpha}(t, t, x)$ of the form

$$
\left[j_{\rho}\right]_{\alpha}\left(t_{1}, x\right) \delta\left(t_{1}-t_{2}\right)
$$

In the Fourier representation, they become

$$
\left[\tilde{j}_{\rho}\right]_{\alpha}\left(\omega_{1}+\omega_{2}, p\right) .
$$

If one wants to calculate only correlation functions of $R=\rho_{1}(t, t, x)+\rho_{2}(t, t, x)$, one needs a source in $t, x$ space that takes the form

$$
j_{\rho}\left(t_{1}, x\right) \delta\left(t_{1}-t_{2}\right)\left(\delta_{\alpha 1}+\delta_{\alpha 2}\right) .
$$

In the Fourier representation, it becomes

$$
\tilde{j}_{\rho}\left(\omega_{1}+\omega_{2}, p\right)\left(\delta_{\alpha 1}+\delta_{\alpha 2}\right)
$$

This somewhat simplifies the calculation.

\subsection{The $\langle(\bar{\psi} \psi) \psi \bar{\psi}\rangle$ vertex function}

Though ultimately we want to calculate the $R$ two-point function, we have first to calculate the connected three-point correlation function with one $R$ insertion,

$$
W^{(1,2)}(x ; y, z)=\langle\bar{\psi}(x) \cdot \psi(x) \psi(y) \cdot \bar{\psi}(z)\rangle_{\mathrm{c}}
$$

because, unlike the $R$ two-point function, it satisfies an integral equation for $N$ large, as the analysis of section 5 shows.

We denote its Fourier transform by $\tilde{W}^{(1,2)}(k ; \ell-k / 2, \ell+k / 2)$ and it is related to the corresponding vertex function by

$$
\tilde{W}^{(1,2)}\left(k ; \ell-\frac{1}{2} k, \ell+\frac{1}{2} k\right)=-\tilde{W}^{(2)}\left(\ell-\frac{1}{2} k\right) \tilde{\Gamma}^{(1,2)}\left(k ; \ell-\frac{1}{2} k, \ell+\frac{1}{2} k\right) \tilde{W}^{(2)}\left(\ell+\frac{1}{2} k\right) .
$$

An important property, which we use systematically later, is the following: one verifies that the vertex function $\tilde{\Gamma}^{(1,2)}$ has the following general decomposition:

$$
\tilde{\Gamma}^{(1,2)}(k ; \ell-k / 2, \ell+k / 2)=-E(k ; \ell-k / 2, \ell+k / 2)-i \sigma_{3} F(k ; \ell-k / 2, \ell+k / 2),
$$

where $E$ and $F$ are scalar functions. 

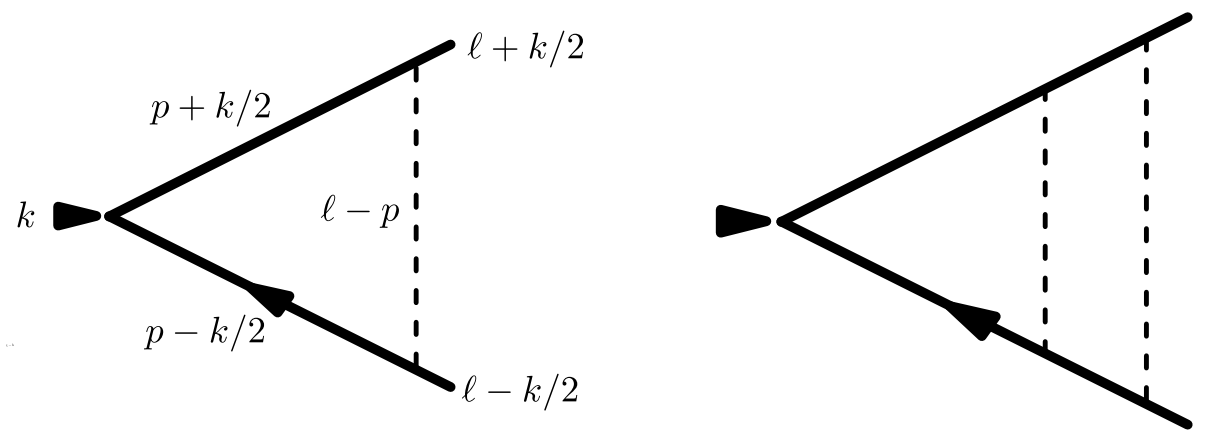

Figure 4. One-loop and two-loop contributions to the $\langle(\bar{\psi} \psi) \psi \bar{\psi}\rangle$ vertex function with dressed propagator. Dotted lines represent gauge fields.

The $R(x)=\bar{\psi}(x) \cdot \psi(x)$ insertion at zero momentum. A differentiation of the fermion two-point function (3.40) with respect to the bare mass $M_{0}$ yields the vertex function (5.7) with an operator insertion at zero momentum. Using the gap equation (7.1), one finds that the mass insertion is given by

$$
\begin{aligned}
& \frac{1}{\left(1-2 g M \Omega_{2}\right)} \frac{\partial \tilde{\Gamma}^{(2)}(\ell)}{\partial M} \\
& =-\frac{1}{\left(1-2 g M \Omega_{2}\right)} U^{-2}\left(\ell_{3}\right)\left[1+2 i g M \sigma_{3}\left(M+i \ell_{3} \sigma_{3}\right) \Xi\left(0, \ell_{3}\right)\right] \\
& =-\frac{1}{\left(1-2 g M \Omega_{2}\right)} U^{-2}\left(\ell_{3}\right)\left[1+\frac{g}{4 \pi} \ell_{3} \frac{i M \sigma_{3}-\ell_{3}}{\ell_{3}^{2}+M^{2}}\right] .
\end{aligned}
$$

The result is consistent with the decomposition (5.7) and one immediately infers the values

$$
\begin{aligned}
& E(0 ; \ell, \ell)=\frac{1}{1-2 g M \Omega_{2}(M)} U^{-2}\left(\ell_{3}\right)\left(1-\frac{g}{4 \pi} \frac{\ell_{3}^{2}}{\ell_{3}^{2}+M^{2}}\right) \\
& F(0 ; \ell, \ell)=\frac{1}{1-2 g M \Omega_{2}(M)} U^{-2}\left(\ell_{3}\right) \frac{g}{4 \pi} \frac{M}{\ell_{3}^{2}+M^{2}} .
\end{aligned}
$$

\subsection{The perturbative expansion of the $\langle(\bar{\psi} \psi) \psi \bar{\psi}\rangle$ vertex function at two loops}

To gather some insight about the general structure of correlation functions involving the $\bar{\psi} \psi$ operator, we begin with a perturbative calculation for $N$ large of the $\langle(\bar{\psi} \psi) \psi \bar{\psi}\rangle$ vertex function $\tilde{\Gamma}^{(1,2)}$. However, we have learned in section 5 that, for $N$ large, the perturbative expansion reduces to a sum of ladder diagrams (figure 4) with the dressed propagator (3.39).

In the decomposition (5.7) the functions $E$ and $F$ have a perturbative expansion of the form

$$
E=\sum_{n=0} E_{n} g^{n}, \quad F=\sum_{n=0} F_{n} g^{n}
$$

with

$$
E_{0}=1, \quad F_{0}=0
$$


We introduce the compact notation

$$
\begin{array}{ll}
D p_{1}=(p+k / 2)^{2}+M^{2}, & D m_{1}=(p-k / 2)^{2}+M^{2}, \\
D p_{2}=(q+k / 2)^{2}+M^{2}, & D m_{2}=(q-k / 2)^{2}+M^{2} .
\end{array}
$$

Recursion relation. Since the perturbative expansion reduces to a sum of ladder diagrams, it is possible to generate them by a recursion formula (see section 5 ). It is given by a $2 \times 2$ matrix acting on the vector $\left(E_{n}, F_{n}\right)$ :

$$
\left(E_{n+1}, F_{n+1}\right)=\mathbf{T}\left(E_{n}, F_{n}\right),
$$

which corresponds to adding two dressed fermion propagators and a gauge propagator to a diagram.

The functions $\left(E_{n}, F_{n}\right)$ depend only on $k$ and on $p_{3}$, if we denote by $p$ the additional integration variables. For $g=0$ (i.e., with the free propagator), we can write the matrix (but a kernel in $\left.\left(p_{3}, \ell_{3}\right)\right)$ as

$$
\mathbf{T}\left(k ; \ell_{3}, p_{3}\right)=\frac{1}{(2 \pi)^{3}} \int \frac{\mathrm{d}^{2} p}{D p_{1} D m_{1}\left(\ell_{3}-p_{3}\right)} \mathcal{T}(k, p)
$$

with

$$
\mathcal{T}(k, p)=\left[\begin{array}{cc}
-2 M p_{3} & \frac{1}{2}\left(D p_{1}+D m_{1}\right)-2 t_{2}-2 p_{3}^{2} \\
-\frac{1}{2}\left(D p_{1}+D m_{1}\right)+2 t_{1} & 2 M p_{3}
\end{array}\right],
$$

where we have set

$$
t_{1}=\frac{1}{4}\left(k^{2}+4 M^{2}\right), t_{2}=\frac{1}{4}\left(k^{2}-k_{3}^{2}\right) .
$$

Terms proportional to $\left(k_{1} p_{2}-k_{2} p_{1}\right)$, with a vanishing integral due to rotation symmetry in the $(1,2)$ plane, have been omitted.

It is useful to decompose $\mathbf{T}$ into the sum of two terms,

$$
\mathbf{T}=\mathbf{T}_{1}+\mathbf{T}_{2}
$$

with, correspondingly,

$$
\mathcal{T}_{2}(k, p)=\frac{1}{2} i \sigma_{2}\left(D p_{1}+D m_{1}\right)
$$

and

$$
\mathcal{T}_{1}\left(k, p_{3}\right)=2\left[\begin{array}{cc}
-M p_{3} & -t_{2}-p_{3}^{2} \\
t_{1} & M p_{3}
\end{array}\right] .
$$

Note that $\mathcal{T}_{1}\left(k, p_{3}\right)$ depends only on the component $p_{3}$.

With the dressed propagator, $\mathbf{T}_{2}$ is not modified. Quite generally, we define

$$
\chi\left(\ell_{3}\right)=g\left[\Theta\left(\ell_{3}+k_{3} / 2\right)+\Theta\left(\ell_{3}-k_{3} / 2\right)\right]
$$

and, moreover, here we set

$$
\chi_{1} \equiv \chi\left(p_{3}\right) .
$$


We can then write the dressed matrix (5.14) as

$$
\begin{aligned}
\mathcal{T}_{1}= & -i \sigma_{2}\left(t_{1}+t_{2}+p_{3}^{2}\right)+\left(t_{1}-t_{2}-p_{3}^{2}\right)\left(\begin{array}{cc}
\sin \chi_{1} & \cos \chi_{1} \\
\cos \chi_{1} & -\sin \chi_{1}
\end{array}\right) \\
& +2 M p_{3}\left(\begin{array}{cc}
-\cos \chi_{1} & \sin \chi_{1} \\
\sin \chi_{1} & \cos \chi_{1}
\end{array}\right) .
\end{aligned}
$$

or

$$
\mathcal{T}_{1}=\mathcal{O}\left(-\chi_{1} / 2\right)\left[\left(t_{1}-t_{2}-p_{3}^{2}\right) \sigma_{1}-i\left(t_{1}+t_{2}+p_{3}^{2}\right) \sigma_{2}-2 M p_{3} \sigma_{3}\right] \mathcal{O}\left(\chi_{1} / 2\right),
$$

where $\mathcal{O}$ is the rotation matrix

$$
\mathcal{O}(\chi)=\left(\begin{array}{cc}
\cos \chi & -\sin \chi \\
\sin \chi & \cos \chi
\end{array}\right) \equiv \mathrm{e}^{-i \sigma_{2} \chi} .
$$

Definitions. The calculations that follow involve only two new basic functions. We first define

$$
\begin{aligned}
\mathcal{B}_{1}(k) & =\int \frac{\mathrm{d}^{3} p}{(2 \pi)^{3}} \frac{1}{\left(p^{2}+M^{2}\right)\left[(k-p)^{2}+M^{2}\right]} \\
& =\int \frac{\mathrm{d}^{3} q}{(2 \pi)^{3}} \int_{0}^{1} \mathrm{~d} s \frac{1}{\left[q^{2}+s(1-s) k^{2}+M^{2}\right]^{2}} \\
& =\frac{1}{4 \pi} \frac{1}{|k|} \arctan \left(\left|\frac{k}{2 M}\right|\right) .
\end{aligned}
$$

which is a one-loop scalar function. Note that in three dimensions all one-loop diagrams can be reduced to elementary functions.

We also define

$$
\Xi(\omega, k)=\frac{1}{(2 \pi)^{3}} \int \frac{\mathrm{d}^{3} p}{\left(\omega-p_{3}\right)\left[(p+k / 2)^{2}+M^{2}\right]\left[(p-k / 2)^{2}+M^{2}\right]} .
$$

It satisfies the relations

$$
\begin{aligned}
\Xi(k, \omega) & =-\Xi(k,-\omega), \\
\Xi(0, \omega) & =-\frac{1}{2 M} \frac{\partial \Theta(\omega)}{\partial M}=\frac{\omega}{8 \pi M} \frac{1}{\omega^{2}+M^{2}}, \\
\Theta\left(\omega-\frac{1}{2} k_{3}\right)-\Theta\left(\omega+\frac{1}{2} k_{3}\right) & =\left.2 k_{3}\left[\omega \Xi(k, \omega)-\mathcal{B}_{1}(k)\right]\right|_{k_{1}=k_{2}=0} .
\end{aligned}
$$

One-loop contribution. We need only the free fermion propagator and, thus, the matrix (5.10) since at one-loop order the phase $\chi=O(g)$ does not contribute.

The one-loop integrand is obtained by acting with $\mathbf{T}$ on the vector $(1,0)$. One finds

$$
\begin{aligned}
E_{1} & =-\frac{1}{(2 \pi)^{3}} \int \frac{\mathrm{d}^{3} p 2 M p_{3}}{\left(\ell_{3}-p_{3}\right) D m_{1} D p_{1}} \\
F_{1} & =\frac{1}{(2 \pi)^{3}} \int \frac{\mathrm{d}^{3} p\left(4 t_{1}-D p_{1}-D m_{1}\right)}{2\left(\ell_{3}-p_{3}\right) D m_{1} D p_{1}} .
\end{aligned}
$$


Integrating, one obtains

$$
E_{1}=-2 M \ell_{3} \Xi\left(k, \ell_{3}\right)+2 M \mathcal{B}_{1}(k)
$$

and

$$
F_{1}=2 t_{1} \Xi\left(k, \ell_{3}\right)-\frac{1}{2}\left[\Theta\left(\ell_{3}+k_{3} / 2\right)+\Theta\left(\ell_{3}-k_{3} / 2\right)\right],
$$

where we have used the definitions (5.19), (5.20) and the notation (5.11). We note that in $F_{1}$ the second term comes from the matrix (5.13).

This result suggests the factorization

$$
\begin{aligned}
\tilde{\Gamma}^{(1,2)}(k ; \ell-k / 2, \ell+k / 2)= & -U^{-1}\left(\ell_{3}-k_{3} / 2\right) \tilde{V}^{(1,2)}(k ; \ell-k / 2, \ell+k / 2) \\
& \times U^{-1}\left(\ell_{3}+k_{3} / 2\right),
\end{aligned}
$$

where at one-loop order $\tilde{V}^{(1,2)}$ reduces to

$$
\tilde{V}^{(1,2)}=1+2 g M\left[\mathcal{B}_{1}(k)-\ell_{3} \Xi\left(\ell_{3}, k\right)\right]+\frac{1}{2} i \sigma_{3} g\left(k^{2}+4 M^{2}\right) \Xi\left(\ell_{3}, k\right)+O\left(g^{2}\right) .
$$

The connected function is then given by

$$
\begin{aligned}
& \tilde{W}^{(1,2)}\left(k ; \ell-\frac{1}{2} k, \ell+\frac{1}{2} k\right)=-\tilde{W}^{(2)}\left(\ell-\frac{1}{2} k\right) \tilde{\Gamma}^{(1,2)}\left(k ; \ell-\frac{1}{2} k, \ell+\frac{1}{2} k\right) \tilde{W}^{(2)}\left(\ell+\frac{1}{2} k\right) \\
& =-U\left(\ell_{3}-\frac{1}{2} k_{3}\right) H^{(1,2)}\left(k ; \ell-\frac{1}{2} k, \ell+\frac{1}{2} k\right) U\left(\ell_{3}+\frac{1}{2} k_{3}\right)
\end{aligned}
$$

with

$$
\begin{aligned}
H^{(1,2)}\left(k ; \ell-\frac{1}{2} k, \ell+\frac{1}{2} k\right)= & {\left[i \ell-\frac{1}{2} i \hbar k+M\right]^{-1} \tilde{V}^{(1,2)}\left(k ; \ell-\frac{1}{2} k, \ell+\frac{1}{2} k\right) } \\
& \times\left[i \ell+\frac{1}{2} i \hbar k+M\right]^{-1} .
\end{aligned}
$$

Quite generally, we introduce the decomposition,

$$
\tilde{V}^{(1,2)}(k ; \ell-k / 2, \ell+k / 2)=A\left(\ell_{3}, k\right)+i \sigma_{3} B\left(\ell_{3}, k\right) .
$$

The relation between the vectors $(E, F)$ and $(A, B)$ then is

$$
\left(\begin{array}{l}
E\left(\ell_{3}, k\right) \\
F\left(\ell_{3}, k\right)
\end{array}\right)=\mathcal{O}\left(-\chi\left(\ell_{3}\right) / 2\right)\left(\begin{array}{l}
A\left(\ell_{3}, k\right) \\
B\left(\ell_{3}, k\right)
\end{array}\right)
$$

We expand $A$ and $B$ in powers of $g$,

$$
A=\sum_{n=0} A_{n} g^{n}, B=\sum_{n=0} B_{n} g^{n}
$$

and obtain

$$
A_{0}=1, A_{1}=2 M\left[\mathcal{B}_{1}(k)-\ell_{3} \Xi\left(\ell_{3}, k\right)\right], B_{0}=0, B_{1}=2 t_{1} \Xi\left(\ell_{3}, k\right) .
$$


Two-loop order. The two-loop order is the sum of the contribution obtained by acting with the matrix $\mathbf{T}$ taken at $g=0$ on the vector $\left(E_{1}, F_{1}\right)$ and the contribution of order $g$ of $\mathbf{T}$ acting on $(1,0)$.

Details about the calculation can be found in appendix C. One obtains

$$
\begin{aligned}
E_{2}= & -\frac{1}{8}\left[\Theta\left(\ell_{3}+k_{3} / 2\right)+\Theta\left(\ell_{3}-k_{3} / 2\right)\right]^{2} \\
& +t_{1}\left[\Theta\left(\ell_{3}+k_{3} / 2\right)+\Theta\left(\ell_{3}-k_{3} / 2\right)\right] \Xi\left(\ell_{3}, k\right) \\
& +2\left[\left(M^{2}-t_{1}\right) \ell_{3}^{2}-t_{1} t_{2}\right] \Xi^{2}\left(\ell_{3}, k\right)+2\left(t_{1}+M^{2}\right) \mathcal{B}_{1}^{2}(k), \\
& -4 M^{2} \ell_{3} \mathcal{B}_{1}(k) \Xi\left(\ell_{3}, k\right) \\
F_{2}= & M\left[\Theta\left(\ell_{3}+k_{3} / 2\right)+\Theta\left(\ell_{3}-k_{3} / 2\right)\right]\left[\ell_{3} \Xi\left(\ell_{3}, k\right)-\mathcal{B}_{1}(k)\right] \\
& +4 M t_{1} \Xi\left(\ell_{3}, k\right) \mathcal{B}_{1}(k) .
\end{aligned}
$$

Then, using the relation (5.28), one verifies that all terms proportional to $\Theta$ functions cancel, justifying to two-loop order the transformation (5.24), and one finds

$$
\begin{aligned}
A_{2}= & 2\left[\left(M^{2}-t_{1}\right) \ell_{3}^{2}-t_{1} t_{2}\right] \Xi^{2}\left(\ell_{3}, k\right)+2\left(t_{1}+M^{2}\right) \mathcal{B}_{1}^{2}(k), \\
& -4 M^{2} \ell_{3} \mathcal{B}_{1}(k) \Xi\left(\ell_{3}, k\right) \\
B_{2}= & 4 M t_{1} \Xi\left(\ell_{3}, k\right) \mathcal{B}_{1}(k) .
\end{aligned}
$$

Higher orders. With the definition

$$
\tau=\sqrt{t_{1} t_{2}+\ell_{3}^{2}\left(t_{1}-M^{2}\right)}=\frac{1}{4} \sqrt{k^{4}+k^{2}\left(4 \ell_{3}^{2}+4 M^{2}-k_{3}^{2}\right)-4 M^{2} k_{3}^{2}},
$$

a calculation to order $g^{6}$ (appendix C) then suggests the general form

$$
\begin{aligned}
& A\left(\ell_{3}, k\right)=\frac{\cos \left(2 g \tau \Xi\left(\ell_{3}, k\right)\right)-\left(M \ell_{3} / \tau\right) \sin \left(2 g \tau \Xi\left(\ell_{3}, k\right)\right)}{\cos \left(g k \mathcal{B}_{1}(k)\right)-2(M / k) \sin \left(g k \mathcal{B}_{1}(k)\right)}, \\
& B\left(\ell_{3}, k\right)=\frac{t_{1}}{\tau} \frac{\sin \left(2 g \tau \Xi\left(\ell_{3}, k\right)\right)}{\cos \left(g k \mathcal{B}_{1}(k)\right)-2(M / k) \sin \left(g k \mathcal{B}_{1}(k)\right)} .
\end{aligned}
$$

Using eq. (5.21), one verifies that the coefficients $A$ and $B$ for $k=0$ are consistent with the expressions (5.8).

\subsection{Integral equation}

For $N$ large, the vertex functions are solutions to one-dimensional coupled integral equations, which in terms of the vector $\mathcal{V}_{E F} \equiv(E, F)$ read

$$
\mathcal{V}_{E F}\left(\ell_{3}, k\right)=\mathcal{V}_{0}+\frac{g}{(2 \pi)^{3}} \int \frac{\mathrm{d}^{3} p \mathcal{T}\left(p_{3}, k\right) \mathcal{V}_{E F}\left(p_{3}, k\right)}{\left(\ell_{3}-p_{3}\right) D p_{1} D m_{1}}
$$

with $\mathcal{V}_{0} \equiv\left(E_{0}, F_{0}\right)=(1,0)$ 
Preliminary remark. Acting with $g \mathbf{T}_{2}$ on both sides of the equation, one obtains

$$
\begin{aligned}
& \frac{1}{(2 \pi)^{3}} \int \frac{\mathrm{d}^{3} q g \mathcal{T}_{2}\left(q_{3}, k\right) \mathcal{V}_{E F}\left(q_{3}, k\right)}{\left(\ell_{3}-q_{3}\right) D m_{2} D p_{2}}=\frac{1}{2} i \chi\left(\ell_{3}\right) \sigma_{2} \mathcal{V}_{0} \\
& \quad+\frac{g^{2}}{(2 \pi)^{6}} \frac{i}{2} \sigma_{2} \int \frac{\mathrm{d}^{3} q d^{3} p\left(D m_{2}+D p_{2}\right) \mathcal{T}\left(p_{3}, k\right) \mathcal{V}_{E F}\left(p_{3}, k\right)}{\left(\ell_{3}-q_{3}\right)\left(q_{3}-p_{3}\right) D m_{1} D p_{1} D m_{2} D p_{2}}
\end{aligned}
$$

with the notation (eq. (5.15)),

$$
\chi\left(\ell_{3}\right)=g\left[\Theta\left(\ell_{3}+k_{3} / 2\right)+\Theta\left(\ell_{3}-k_{3} / 2\right)\right] .
$$

The usual identity

$$
\frac{1}{\left(\ell_{3}-q_{3}\right)\left(q_{3}-p_{3}\right)}=\frac{1}{\left(\ell_{3}-q_{3}\right)\left(\ell_{3}-p_{3}\right)}+\frac{1}{\left(p_{3}-q_{3}\right)\left(p_{3}-\ell_{3}\right)}
$$

transforms the equation into

$$
\begin{gathered}
\frac{g}{(2 \pi)^{3}} \int \frac{\mathrm{d}^{3} q \mathcal{T}_{2}\left(q_{3}, k\right) \mathcal{V}_{E F}\left(q_{3}, k\right)}{\left(\ell_{3}-q_{3}\right) D m_{2} D p_{2}}=\frac{i}{2} \chi\left(\ell_{3}\right) \sigma_{2} \mathcal{V}_{E F}\left(\ell_{3}, k\right) \\
-\frac{g}{(2 \pi)^{3}} \frac{i}{2} \sigma_{2} \int \frac{\mathrm{d}^{3} p \chi\left(p_{3}\right) \mathcal{T}\left(p_{3}, k\right) \mathcal{V}_{E F}\left(p_{3}, k\right)}{\left(\ell_{3}-p_{3}\right) D m_{1} D p_{1}}
\end{gathered}
$$

Rescaled integral equation. In the integral equation, we now rescale $\chi \mapsto \varepsilon \chi, \mathcal{T}_{2} \mapsto \varepsilon \mathcal{T}_{2}$ and determine the $\varepsilon$ dependence of the solution. Differentiating with respect to $\varepsilon$, we find

$$
\begin{aligned}
\partial_{\varepsilon} \mathcal{V}_{E F}\left(\ell_{3}, k\right)= & \frac{g}{(2 \pi)^{3}} \int \frac{\mathrm{d}^{3} p \partial_{\varepsilon} \mathcal{T}\left(p_{3}, k\right) \mathcal{V}_{E F}\left(p_{3}, k\right)}{\left(\ell_{3}-p_{3}\right) D m_{1} D p_{1}} \\
& +\frac{g}{(2 \pi)^{3}} \int \frac{\mathrm{d}^{3} p \mathcal{T}\left(p_{3}, k\right) \partial_{\varepsilon} \mathcal{V}_{E F}\left(p_{3}, k\right)}{\left(\ell_{3}-p_{3}\right) D m_{1} D p_{1}}
\end{aligned}
$$

where

$$
\begin{aligned}
\partial_{\varepsilon} \mathcal{T}\left(p_{3}, k\right) & =\frac{1}{2} i \chi\left(p_{3}\right)\left(\sigma_{2} \mathcal{T}_{1}-\mathcal{T}_{1} \sigma_{2}\right)+\mathcal{T}_{2} \\
& =\frac{1}{2} i \chi\left(p_{3}\right)\left(\sigma_{2} \mathcal{T}-\mathcal{T} \sigma_{2}\right)+\mathcal{T}_{2}
\end{aligned}
$$

because $\mathcal{T}_{2}$ commutes with $\sigma_{2}$.

We now use the identity (5.32) (which is not affected by the rescaling) to eliminate $\mathcal{T}_{2}$. The equation becomes

$$
\begin{aligned}
\partial_{\varepsilon} \mathcal{V}_{E F}\left(\ell_{3}, k\right)= & \frac{g}{(2 \pi)^{3}} \int \frac{\mathrm{d}^{3} p \mathcal{T}\left(p_{3}, k\right) \partial_{\varepsilon} \mathcal{V}_{E F}\left(p_{3}, k\right)}{\left(\ell_{3}-p_{3}\right) D m_{1} D p_{1}}+\frac{i}{2} \chi\left(\ell_{3}\right) \sigma_{2} \mathcal{V}_{E F}\left(\ell_{3}, k\right) \\
& +\frac{g}{(2 \pi)^{3}} \frac{i}{2} \int \frac{\mathrm{d}^{3} p \chi\left(p_{3}\right)\left[\sigma_{2} \mathcal{T}\left(p_{3}, k\right)-\mathcal{T}\left(p_{3}, k\right) \sigma_{2}\right] \mathcal{V}_{E F}\left(p_{3}, k\right)}{\left(\ell_{3}-p_{3}\right) D m_{1} D p_{1}} \\
& -\frac{g}{(2 \pi)^{3}} \frac{i}{2} \sigma_{2} \int \frac{\mathrm{d}^{3} p \chi\left(p_{3}\right) \mathcal{T}\left(p_{3}, k\right) \mathcal{V}_{E F}\left(p_{3}, k\right)}{\left(\ell_{3}-p_{3}\right) D m_{1} D p_{1}} \\
= & \frac{g}{(2 \pi)^{3}} \int \frac{\mathrm{d}^{3} p \mathcal{T}\left(p_{3}, k\right) \partial_{\varepsilon} \mathcal{V}_{E F}\left(p_{3}, k\right)}{\left(\ell_{3}-p_{3}\right) D m_{1} D p_{1}}+\frac{i}{2} \chi\left(\ell_{3}\right) \sigma_{2} \mathcal{V}_{E F}\left(\ell_{3}, k\right) \\
& -\frac{g}{(2 \pi)^{3}} \frac{i}{2} \int \frac{\mathrm{d}^{3} p \chi\left(p_{3}\right) \mathcal{T}\left(p_{3}, k\right) \sigma_{2} \mathcal{V}_{E F}\left(p_{3}, k\right)}{\left(\ell_{3}-p_{3}\right) D m_{1} D p_{1}}
\end{aligned}
$$


Setting

$$
X\left(\ell_{3}, k\right)=\partial_{\varepsilon} \mathcal{V}_{E F}\left(\ell_{3}, k\right)-\frac{1}{2} i \chi\left(\ell_{3}\right), \sigma_{2} \mathcal{V}_{E F}\left(\ell_{3}, k\right)
$$

we obtain the equation

$$
X\left(\ell_{3}, k\right)=\frac{g}{(2 \pi)^{3}} \int \frac{\mathrm{d}^{3} p \mathcal{T}\left(p_{3}, k\right) X\left(p_{3}, k\right)}{\left(\ell_{3}-p_{3}\right) D m_{1} D p_{1}},
$$

which has $X=0$ as the only solution expandable in powers of $g$. We infer

$$
\partial_{\varepsilon} \mathcal{V}_{E F}\left(\ell_{3}, k\right)=\frac{1}{2} i \chi\left(\ell_{3}\right) \sigma_{2} \mathcal{V}_{E F}\left(\ell_{3}, k\right)
$$

and thus

$$
\left.\mathcal{V}_{E F}\left(\ell_{3}, k\right)\right|_{\varepsilon=1}=\left.\mathrm{e}^{i \chi\left(\ell_{3}\right) \sigma_{2} / 2} \mathcal{V}_{E F}\left(\ell_{3}, k\right)\right|_{\varepsilon=0} .
$$

We recognize the relation (5.28) and thus

$$
\left.\mathcal{V}_{E F}\left(\ell_{3}, k\right)\right|_{\varepsilon=0}=\left(\begin{array}{l}
A \\
B
\end{array}\right) .
$$

The vector $\mathcal{V}=(A, B)$ is thus solution of eq. (5.31) with $\varepsilon=0$.

\subsection{Solution of the reduced integral equation}

The vector $\mathcal{V}=(A, B)$ is solution of the reduced integral equation

$$
\begin{aligned}
& \mathcal{V}\left(\ell_{3}, k\right) \\
& =(1,0)+\frac{g}{(2 \pi)^{3}} \int \frac{\mathrm{d}^{3} p \mathcal{T}_{1}\left(p_{3}, k\right) \mathcal{V}\left(p_{3}, k\right)}{\left(\ell_{3}-p_{3}\right)\left[(p+k / 2)^{2}+M^{2}\right]\left[(p-k / 2)^{2}+M^{2}\right]}
\end{aligned}
$$

or in component form,

$$
\begin{aligned}
& A\left(\ell_{3}, k\right)=1-\frac{2 g}{(2 \pi)^{3}} \int \frac{\mathrm{d}^{3} p\left[M p_{3} A\left(p_{3}, k\right)+\left(p_{3}^{2}+t_{2}\right) B\left(p_{3}, k\right)\right]}{\left(\ell_{3}-p_{3}\right)\left[(p+k / 2)^{2}+M^{2}\right]\left[(p-k / 2)^{2}+M^{2}\right]}, \\
& B\left(\ell_{3}, k\right)=\frac{2 g}{(2 \pi)^{3}} \int \frac{\mathrm{d}^{3} p\left[t_{1} A\left(p_{3}, k\right)+M p_{3} B\left(p_{3}, k\right)\right]}{\left(\ell_{3}-p_{3}\right)\left[(p+k / 2)^{2}+M^{2}\right]\left[(p-k / 2)^{2}+M^{2}\right]} .
\end{aligned}
$$

Inserting the expressions $(5.30 \mathrm{a}),(5.30 \mathrm{~b})$ in the equations, we note that the numerators simplify since

$$
\begin{aligned}
M p_{3} A\left(p_{3}, k\right)+\left(p_{3}^{2}+t_{2}\right) B\left(p_{3}, k\right) & =\frac{M p_{3} \cos \left(2 g \tau \Xi\left(p_{3}, k\right)\right)+\tau \sin \left(2 g \tau \Xi\left(p_{3}, k\right)\right)}{\cos \left(g k \mathcal{B}_{1}(k)\right)-2(M / k) \sin \left(g k \mathcal{B}_{1}(k)\right)}, \\
t_{1} A\left(p_{3}, k\right)+M p_{3} B\left(p_{3}, k\right) & =\frac{t_{1} \cos \left(2 g \tau \Xi\left(p_{3}, k\right)\right)}{\cos \left(g k \mathcal{B}_{1}(k)\right)-2(M / k) \sin \left(g k \mathcal{B}_{1}(k)\right)} .
\end{aligned}
$$

The verification that the functions are the solutions of the integral equation is then simple and relies on a few identities derived in B.2 concerning integrals of sine and cosine functions. The proofs of the latter identities rely on an expansion order by order in powers of the coupling $g$. 

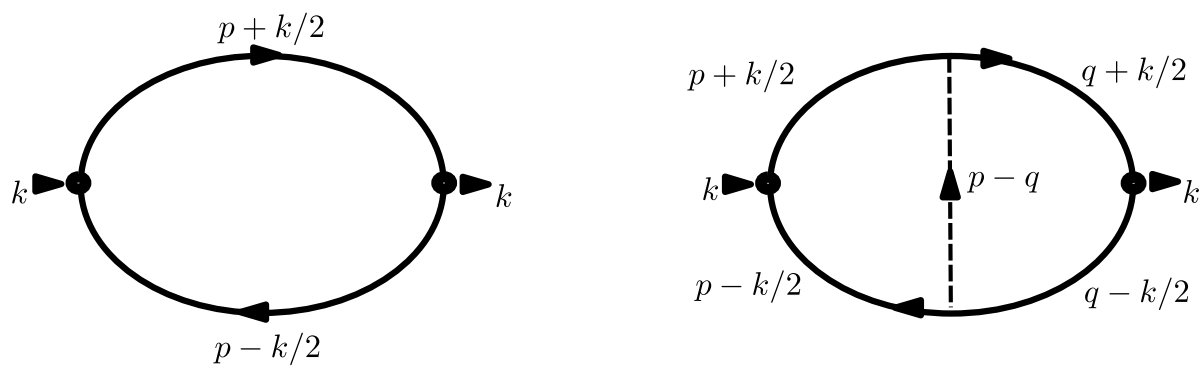

Figure 5. The $(\bar{\psi} \psi)$ two-point function: one- and two-loop diagrams. Dotted lines represent gauge fields.

\section{The $R$ two-point function}

We have defined (eqs. (3.42), (3.43)) the two gauge-invariant operators

$$
R_{\alpha}(t, x)=\frac{1}{N} \bar{\psi}_{\alpha}(t, x) \cdot \psi_{\alpha}(t, x), \quad R(t, x)=R_{1}(t, x)+R_{2}(t, x) .
$$

We have already determined the connected $R$ two-point function $\langle\tilde{R}(k) \tilde{R}(-k)\rangle_{\mathrm{c}}$ at zero momentum (eq. (3.49)). We now calculate it for generic momenta. We begin with a threeloop perturbative calculation in the large $N$ limit and then derive the exact result from the known vertex function.

\subsection{Perturbative calculations}

Since calculations are performed using the dressed fermion propagator (3.39), only ladder diagrams have to be considered (see section 5).

One-loop result. The one-loop diagram (first diagram in figure 5) with the dressed propagator reads

$$
\langle\tilde{R}(k) \tilde{R}(-k)\rangle_{\mathrm{c}}=\frac{1}{(2 \pi)^{3}} \int \frac{\mathrm{d}^{3} p \mathcal{N}(p, k)}{D p_{1} D m_{1}},
$$

where

$$
\mathcal{N}=-\frac{1}{2} k^{2}+2 p^{2}-2 M^{2}-4 M p_{3} \sin \left(\chi_{1}\right)+\frac{1}{2}\left(4 p_{3}^{2}-k_{3}^{2}-4 M^{2}\right)\left(\cos \left(\chi_{1}\right)-1\right)
$$

and $\chi_{1} \equiv \chi\left(p_{3}\right)$ is the angle (5.15).

We note that

$$
2 p^{2}=D p_{1}+D m_{1}-\frac{1}{2} k^{2}-2 M^{2} .
$$

Thus,

$$
\langle\tilde{R}(k) \tilde{R}(-k)\rangle_{\mathrm{c}}=2 \Omega_{1}(M)+\frac{1}{(2 \pi)^{3}} \int \frac{\mathrm{d}^{3} p \mathcal{N}^{\prime}(p, k)}{D p_{1} D m_{1}}
$$

with

$$
\mathcal{N}^{\prime}=-\left(k^{2}+4 M^{2}\right)-4 M p_{3} \sin \left(\chi_{1}\right)+\frac{1}{2}\left(4 p_{3}^{2}-k_{3}^{2}-4 M^{2}\right)\left(\cos \left(\chi_{1}\right)-1\right) .
$$




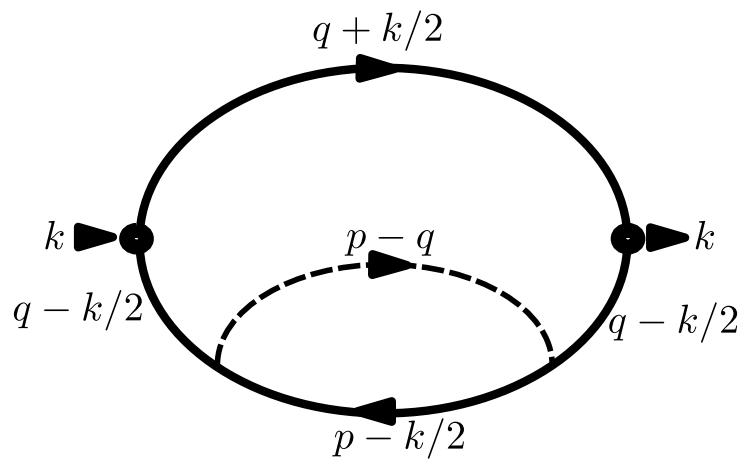

Figure 6. The $\bar{\psi} \psi$ two-point function at two loops. Dotted lines represent gauge fields.

At one-loop order, $\chi_{1}=0$ and, thus, the contribution is

$$
\begin{aligned}
\langle\tilde{R}(k) \tilde{R}(-k)\rangle_{\mathrm{c}} & =2 \Omega_{1}(M)-\frac{1}{(2 \pi)^{3}} \int \frac{\mathrm{d}^{3} p\left(k^{2}+4 M^{2}\right)}{\left[(p+k / 2)^{2}+M^{2}\right]\left[(p-k / 2)^{2}+M^{2}\right]} \\
& =2 \Omega_{1}(M)-\left(k^{2}+4 M^{2}\right) \mathcal{B}_{1}(k) .
\end{aligned}
$$

Two-loop result. At two-loop, one needs the second diagram of figure 5 with the free propagator together with the order $g$ contribution of expression (6.1) (which corresponds to the diagram of figure 6 ). The two-loop ladder diagram yields

$$
\begin{aligned}
& \frac{2 M g}{(2 \pi)^{6}} \int \frac{\mathrm{d}^{3} p \mathrm{~d}^{3} q\left[\left(p_{3}-q_{3}\right)\left(k^{2}+4 M^{2}\right)+q_{3}\left(D p_{1}+D m_{1}\right)-p_{3}\left(D p_{2}+D m_{2}\right)\right]}{\left(q_{3}-p_{3}\right) D p_{1} D m_{1} D p_{2} D m_{2}} \\
& =-2 M g\left(k^{2}+4 M^{2}\right) \mathcal{B}_{1}^{2}(k) \\
& \quad+\frac{2 M g}{(2 \pi)^{6}} \int \frac{\mathrm{d}^{3} p \mathrm{~d}^{3} q\left[q_{3}\left(D p_{1}+D m_{1}\right)-p_{3}\left(D p_{2}+D m_{2}\right)\right]}{\left(q_{3}-p_{3}\right) D p_{1} D m_{1} D p_{2} D m_{2}} \\
& =-2 M g\left(k^{2}+4 M^{2}\right) \mathcal{B}_{1}^{2}(k)+\frac{4 M}{(2 \pi)^{3}} \int \frac{\mathrm{d}^{3} p p_{3} \chi\left(p_{3}\right)}{D p_{1} D m_{1}},
\end{aligned}
$$

where the $(p, q)$ symmetry has been used. The last term is exactly cancelled by the order $g$ of expression (6.1), which yields

$$
-\frac{4 M}{(2 \pi)^{3}} \int \frac{\mathrm{d}^{3} p p_{3} \chi\left(p_{3}\right)}{D p_{1} D m_{1}} .
$$

Adding the two contributions, one finds

$$
-2 M g\left(k^{2}+4 M^{2}\right) \mathcal{B}_{1}^{2}(k) .
$$

Three-loop contribution. Details about the direct three- and four-loop calculations are given in section E. One finds (eqs. (E.6), (E.7))

$$
-\frac{1}{3} g^{2}\left(k^{2}+12 M^{2}\right)\left(k^{2}+4 M^{2}\right) \mathcal{B}_{1}^{3}(k)-\frac{4}{3} g^{3} M\left(k^{2}+4 M^{2}\right)\left(k^{2}+6 M^{2}\right) \mathcal{B}_{1}^{4}(k) .
$$




\section{2 $R$ two-point function and vertex three-point function}

The $R$ two-point function is derived from the vertex three point function (5.7) by multiplying it by two additional dressed fermion propagators and taking the trace. This leads to the general relation

$$
\langle\tilde{R}(k) \tilde{R}(-k)\rangle_{\mathrm{c}}=2 \Omega_{1}(M)-\frac{1}{(2 \pi)^{3}} \int \frac{\mathrm{d}^{3} q \mathcal{N}\left(q_{3}, k\right)}{D m_{2} D p_{2}}
$$

with

$$
\begin{aligned}
\mathcal{N}= & D m_{2}+D p_{2}+\left[\left(4 t_{1}-D m_{2}-D p_{2}\right) \cos \left(\chi_{2} / 2\right)+4 M q_{3} \sin \left(\chi_{2} / 2\right)\right] A\left(q_{3}, k\right) \\
& +4\left[M q_{3} \cos \left(\chi_{2} / 2\right)+\left(t_{2}+q_{3}^{2}-\frac{1}{4}\left(D m_{2}+D p_{2}\right)\right) \sin \left(\chi_{2} / 2\right)\right] B\left(q_{3}, k\right)
\end{aligned}
$$

where $\chi_{2} \equiv \chi\left(q_{3}\right)$ (definition (5.15)), $A$ and $B$ are the coefficients of $\mathbf{1}$ and $i \sigma_{3}$ in the vertex function $\tilde{V}^{1,2)}$ (the relation (5.28) between $(E, F)$ and $(A, B)$ has been used).

We first prove phase factor factorization and cancellation, in such a way that the relation $(6.2)$ reduces to

$$
\begin{aligned}
& \langle\tilde{R}(k) \tilde{R}(-k)\rangle_{\mathrm{c}}=2 \Omega_{1}(M) \\
& -\frac{1}{(2 \pi)^{3}} \int \frac{\mathrm{d}^{3} q\left[\left(k^{2}+4 M^{2}\right) A\left(q_{3}, k\right)+4 M q_{3} B\left(q_{3}, k\right)\right]}{\left[(q+k / 2)^{2}+M^{2}\right]\left[(q-k / 2)^{2}+M^{2}\right]},
\end{aligned}
$$

where $A$ and $B$ are the coefficients of $\mathbf{1}$ and $i \sigma_{3}$ in $\tilde{V}^{(1,2)}$ given by equations (5.30a), (5.30b).

Proof at first order. The first contribution of the additional terms in eq. (6.2) with respect to $(6.4)$ is

$$
\begin{aligned}
& \int \frac{\mathrm{d}^{3} q}{(2 \pi)^{3}} \frac{\left[A\left(q_{3}, k\right)-1\right]\left(D p_{2}+D m_{2}\right)}{D m_{2} D p_{2}} \\
& -2 \int \frac{\mathrm{d}^{3} q}{(2 \pi)^{3}} \frac{\chi_{2}\left[M q_{3} A\left(q_{3}, k\right)+\left(t_{2}+q_{3}^{2}\right) B\left(q_{3}, k\right)\right]}{D m_{2} D p_{2}} .
\end{aligned}
$$

We transform the first term by using the integral equation (5.34a), (5.34b):

$$
\begin{aligned}
& \int \frac{\mathrm{d}^{3} q}{(2 \pi)^{3}} \frac{\left[A\left(q_{3}, k\right)-1\right]\left(D p_{2}+D m_{2}\right)}{D m_{2} D p_{2}} \\
& =-\frac{2 g}{(2 \pi)^{6}} \int \frac{\mathrm{d}^{3} p \mathrm{~d}^{3} q\left(D p_{2}+D m_{2}\right)\left[M p_{3} A\left(p_{3}, k\right)+\left(p_{3}^{2}+t_{2}\right) B\left(p_{3}, k\right)\right]}{D p_{1} D m_{1} D p_{2} D m_{2}\left(q_{3}-p_{3}\right)} .
\end{aligned}
$$

Integrating over $q$, we find

$$
\frac{1}{(2 \pi)^{3}} \int \frac{\mathrm{d}^{3} p \chi\left(p_{3}\right)\left[2 M p_{3} A\left(p_{3}, k\right)+2\left(p_{3}^{2}+t_{2}\right) B\left(p_{3}, k\right)\right]}{D p_{1} D m_{1}}
$$

and, thus, the first order difference vanishes. 
A general identity. For the general proof, we need

$$
\mathcal{I}_{n}\left(p_{3}\right)=g \int \frac{\mathrm{d}^{3} q}{(2 \pi)^{3}} \frac{\left(D p_{2}+D m_{2}\right) \chi^{n}\left(q_{3}\right)}{\left(p_{3}-q_{3}\right) D p_{2} D m_{2}} .
$$

We introduce the integral representation of $\chi\left(q_{3}\right)$,

$$
\chi\left(q_{3}\right)=g \int \frac{\mathrm{d}^{3} r^{i}}{(2 \pi)^{3}} \frac{\left(D p_{i}+D m_{i}\right)}{\left(q_{3}-r_{3}^{i}\right) D p_{i} D m_{i}}
$$

with $i>2$ and

$$
D p_{i}=\left(r^{i}+k / 2\right)^{2}+M^{2}, D m_{i}=\left(r^{i}-k / 2\right)^{2}+M^{2} .
$$

The integral becomes

$$
\mathcal{I}_{n}=g^{n+1} \int \frac{\mathrm{d}^{3} q}{(2 \pi)^{3}} \frac{\left(D p_{2}+D m_{2}\right)}{\left(p_{3}-q_{3}\right) D p_{2} D m_{2}} \prod_{i=3}^{n+2} \frac{\mathrm{d}^{3} r^{i}}{(2 \pi)^{3}} \frac{\left(D p_{i}+D m_{i}\right)}{\left(q_{3}-r_{3}^{i}\right) D p_{i} D m_{i}} .
$$

Except for the gauge propagator, the integrand in symmetric in $\left(q, r^{i}\right)$. Symmetrizing the whole integrand and using the identity (B.2), we find

$$
\mathcal{I}_{n}\left(p_{3}\right)=\frac{1}{(n+1)} \chi^{n+1}\left(p_{3}\right) .
$$

General proof. We now consider the whole contribution coming from the numerator (6.3) and proportional to $\left(D p_{2}+D m_{2}\right)$,

$$
\left(D p_{2}+D m_{2}\right)\left[1-\cos \left(\chi_{2} / 2\right) A\left(q_{3}, k\right)-\sin \left(\chi_{2} / 2\right) B\left(q_{3}, k\right)\right] .
$$

We again use the integral equation (5.34a), (5.34b) both for $A$ and $B$ and obtain

$$
\left(D p_{2}+D m_{2}\right)\left[1-\cos \left(\chi_{2} / 2\right)+\frac{2 g}{(2 \pi)^{3}} \int \frac{\mathrm{d}^{3} p \mathcal{N}^{\prime}\left(p_{3}, k\right)}{\left(q_{3}-p_{3}\right) D p_{1} D m_{1}}\right]
$$

with

$$
\begin{aligned}
\mathcal{N}^{\prime}\left(p_{3}, k\right)= & {\left[M p_{3} A\left(p_{3}, k\right)+\left(p_{3}^{2}+t_{2}\right) B\left(p_{3}, k\right)\right] \cos \left(\chi_{2} / 2\right) } \\
& \left.+\left[t_{1} A\left(p_{3}, k\right)+M p_{3} B\left(p_{3}, k\right)\right] \sin \left(\chi_{2}\right) / 2\right) .
\end{aligned}
$$

We substitute this expression into eq. (6.2), integrate over $q$ and find the numerator

$$
\begin{array}{r}
-4\left[M p_{3} A\left(p_{3}, k\right)+\left(p_{3}^{2}+t_{2}\right) B\left(p_{3}, k\right)\right] \sin \left(\chi_{1} / 2\right) \\
-4\left[t_{1} A\left(p_{3}, k\right)+M p_{3} B\left(p_{3}, k\right)\right]\left[\cos \left(\chi_{1} / 2\right)-1\right]
\end{array}
$$

with a denominator $D p_{1} D m_{1}$. We have still to add the remaining part of (6.3) (replacing $q$ by $p$ as integration variable),

$$
\begin{aligned}
& {\left[4 t_{1} \cos \left(\chi_{1} / 2\right)+4 M p_{3} \sin \left(\chi_{1} / 2\right)\right] A\left(p_{3}, k\right)} \\
& \quad+\left[4 M p_{3} \cos \left(\chi_{1} / 2\right)+\left(4 t_{2}+4 p_{3}^{2}\right) \sin \left(\chi_{1} / 2\right)\right] B\left(p_{3}, k\right) .
\end{aligned}
$$

The $\chi$-dependence cancels and one recovers the anticipated expression (6.4),

$$
4\left[t_{1} A\left(p_{3}, k\right)+M p_{3} B\left(p_{3}, k\right)\right] .
$$




\subsection{The exact $R$ two-point function at large $N$}

We now derive the connected two-point function $\langle\tilde{R}(k) \tilde{R}(-k)\rangle_{\mathrm{c}}$ from the expression $(5.30 \mathrm{a}),(5.30 \mathrm{~b})$ of the vertex function. In section 6.2 , we have proved the reduced relation (6.4),

$$
\begin{aligned}
& \langle\tilde{R}(k) \tilde{R}(-k)\rangle_{\mathrm{c}}=2 \Omega_{1}(M) \\
& -\frac{1}{(2 \pi)^{3}} \int \frac{\mathrm{d}^{3} q\left[\left(k^{2}+4 M^{2}\right) A\left(q_{3}, k\right)+4 M q_{3} B\left(q_{3}, k\right)\right]}{\left[(q+k / 2)^{2}+M^{2}\right]\left[(q-k / 2)^{2}+M^{2}\right]},
\end{aligned}
$$

where $A$ and $B$ are the coefficients of $\mathbf{1}$ and $i \sigma_{3}$ in $\tilde{V}^{(1,2)}$ given by equations (5.30a), (5.30b).

Inserting into the equation the expressions (5.30a), (5.30b), one finds a factor

$$
-\frac{k^{2}+4 M^{2}}{\cos \left(g k \mathcal{B}_{1}(k)\right)-2(M / k) \sin \left(g k \mathcal{B}_{1}(k)\right)}
$$

multiplied by the integral

$$
\int \frac{\mathrm{d}^{3} q \cos \left(2 g \tau \Xi\left(q_{3}, k\right)\right)}{\left[(q+k / 2)^{2}+M^{2}\right]\left[(q-k / 2)^{2}+M^{2}\right]} .
$$

Using the result (B.7), one obtains the two-point function

$$
\langle\tilde{R}(k) \tilde{R}(-k)\rangle_{\mathrm{c}}=2 \Omega_{1}(M)-\frac{k^{2}+4 M^{2}}{k g} \frac{\tan \left(g k \mathcal{B}_{1}(k)\right)}{1-2 M \tan \left(g k \mathcal{B}_{1}(k)\right) / k},
$$

where from (5.19),

$$
g k \mathcal{B}_{1}(k)=\frac{g}{4 \pi} \arctan (k / 2 M) .
$$

This expression agrees with the known values at $k=0$ (eq. (3.49)) and $M=0$ and the perturbative expansion to the calculated orders. It requires only a constant additive renormalization.

\section{$7 \quad$ Mass gap and critical coupling}

Mass gap equation. We now examine the gap equation (3.29),

$$
M_{0}=M+g \Omega_{1}(M),
$$

where $M$ is the fermion physical mass and $\Omega_{1}(M)$ is a divergent quantity defined by equation (3.28).

Introducing the explicit result (3.34), we infer from eq. (7.1) that $M_{0}$ can be written as

$$
M_{0}=M_{c}+m
$$

with

$$
M_{c}=g \frac{\Lambda}{4 \pi}
$$


where $m$, which is finite for $\Lambda \rightarrow \infty$ since $M$ is finite, provides a physical mass scale. The gap equation (7.1) then reads

$$
m=M\left(1-\frac{g}{4 \pi} \operatorname{sgn}(M)\right)
$$

which has solutions in the domain connected with $g=0$ only for $|g|<4 \pi$.

We note that if $M$ is a solution, $-M$ is a solution of the gap equation obtained by changing $M_{0} \rightarrow-M_{0}$ together with $g \rightarrow-g$, a general symmetry of the problem. Therefore, from now on we restrict the discussion to $M \geq 0$.

For $g \neq 4 \pi$, the fermion mass, solution of the gap equation, is

$$
M=\frac{m}{1-g / 4 \pi} .
$$

If $m \neq 0$ the fermions are massive and scale symmetry is explicitly broken. For $m>0$, the equation has a solution only for $g<4 \pi$ while for $m<0$ it has a solution only for $g>4 \pi$. For $m \neq 0$, since $M$ diverges at $g=4 \pi$, crossing the values $g= \pm 4 \pi$ seems impossible and one may suspect that the field theory makes sense only for $|g|<4 \pi$.

For the special value $m=0$ or $M_{0}=M_{c}$, eq. (7.4) reduces to

$$
M=\frac{g}{4 \pi}|M| \text {. }
$$

For $g \neq g_{c}= \pm 4 \pi$, the equation implies $M=0$, that is, fermions are massless and the theory is thus conformal invariant.

By contrast, for $m=0$ and $g= \pm 4 \pi$, the equation is always satisfied and $M$ remains undetermined. We then note that if indeed $M \neq 0$ in a theory with no renormalized mass scale, $m=0$ indicates a spontaneous breaking of scale invariance. As in similar cases (like in $[22]$ and earlier works $[18,19])$ it requires the appearance of a dilaton pole at $g= \pm 4 \pi$. This issue is discussed further below where we show that no dilaton pole appears, yet another indication that, indeed, the physical range is limited to $-4 \pi \leq g \leq 4 \pi$.

Returning to the definition (2.16) of the coupling constant $g$, one verifies that the critical values $g_{c}$ are acceptable since they imply the relation between integers

$$
\kappa= \pm N
$$

The massless or large momentum limit. In the limit $M \rightarrow 0$ or, equivalently, $k \rightarrow \infty$, the expression (6.6) reduces to

$$
\langle\tilde{R}(k) \tilde{R}(-k)\rangle_{\mathrm{c}} \underset{M=0}{=} \frac{\Lambda}{2 \pi}-\frac{\tan (g / 8)}{g} k,
$$

which, up to a constant, is simply proportional to the two-fermion phase space. For all values of $k$, the correlation function diverges for $g= \pm 4 \pi$ and for $4 \pi<|g|<8 \pi$ it violates positivity. Therefore, in the domain containing the origin, the physical range is limited to $|g| \leq 4 \pi$. The special case $|g|=4 \pi$ has to be examined separately.

Eq. (7.7) is in agreement with ref. [15] where calculations were done in the light-cone gauge (See eqs. (32) and (33) in ref. [15]). A detailed analysis of this result in different regularization schemes in the light-cone gauge can be found in the appendix of ref. [23]. 
Singularity of the two-point function and critical couplings. It is now interesting to look at the zeros of the denominator in expression (6.6). The denominator vanishes for

$$
\tan \left(\frac{g}{4 \pi} \arctan (k / 2 M)\right)=\frac{k}{2 M}
$$

and, thus,

$$
\frac{g}{4 \pi} \arctan (k / 2 M)=\arctan (k / 2 M) \quad(\bmod \pi) .
$$

The denominator vanishes for all values of $k$ for $g=4 \pi$, that is, for one critical value of $g$ that is singled out by the fermion gap equation. More precisely,

$$
\langle\tilde{R}(k) \tilde{R}(-k)\rangle_{\mathrm{c}} \underset{g \rightarrow 4 \pi}{\sim}-\frac{1}{(4 \pi-g)} \frac{k}{\arctan (k / 2 M)} .
$$

This expression shows that even after a renormalization of the $R$-field to remove the singularity at $g=4 \pi$, the $R$ two-point function has no massless pole at $k=0$.

Other solutions are $k$-dependent. Another family of solutions is given by

$$
\frac{g}{4 \pi}-1=-\frac{\pi}{\arctan (k / 2 M)}
$$

which, for $k \rightarrow \infty$ converge toward $g=-4 \pi$ from below. Since in the Euclidean formulation poles with $k$ real are unphysical, this confirms again that the admissible range of values of $g$ is restricted to $-4 \pi \leq g \leq 4 \pi$. In this range, the physical fermion mass is always an increasing function of the bare mass (eq. (7.4)), which sounds reasonable.

We thus note that the case $m=M_{0}-M_{c}=0$ and $g= \pm 4 \pi$, which allowed for a possible non vanishing fermion mass $M$, is inconsistent. Such a spontaneously broken scale invariance phase would require the appearance of a massless pole in eq. (6.6). However, this does not happen. After a renormalization of the $R$-field by a factor $\sqrt{4 \pi-g}$ to remove the singularity at $g=4 \pi$, one verifies from eq. (7.8) that the correlation function has no pole at $k^{2}=0$. Moreover, expression (3.50) immediately shows that this is not a suitable renormalization to remove singularities at $g=4 \pi$. A more suitable renormalization would seem to be a multiplication by $(4 \pi-g)$ but then the two-point function, as well as the three-point function at zero-momentum, vanish in the $g=4 \pi$ limit.

\section{The current two-point function}

The current associated to fermion number conservation is

$$
J_{\mu}(x)=i \bar{\psi}(x) \sigma_{\mu} \cdot \psi(x)
$$

The current is gauge-invariant and conserved.

The third component is related to quantities already defined since

$$
J_{3}(x)=i\left(R_{1}(x)-R_{2}(x)\right)
$$




\subsection{The $\left\langle J_{3} \psi \bar{\psi}\right\rangle$ vertex}

Following the same strategy as for the $R$ two-point function, we first determine the $\left\langle J_{3} \psi \bar{\psi}\right\rangle$ vertex, which again has a simple decomposition of the form (5.7). It satisfies the integral equation (5.31) but with the different initial conditions

$$
E_{0}=0, \quad F_{0}=1
$$

The proof of section 5.5 does not depend on the explicit form of the inhomogeneous term and, therefore, the vertex function factorizes as in expression (5.24),

$$
\left\langle J_{3} \psi \bar{\psi}\right\rangle=U^{-1} V_{3}^{(1,2)} U^{-1},
$$

where $V_{3}^{(1,2)}$ can be written as

$$
\tilde{V}_{3}^{(1,2)}=A+i \sigma_{3} B \text { with } A=\sum_{n=1} A_{n} g^{n}, B=1+\sum_{n=1} B_{n} g^{n}
$$

and, in Fourier representation, the vector $\mathcal{V}_{3} \equiv(A, B)$ satisfies the reduced integral equation $(5.33)$ but with the vector $(1,0)$ replaced by the vector $(0,1)$.

\subsection{Perturbative calculations}

To gain some insight about the structure of the current vertex function, we calculate a few terms of the perturbative expansion.

One- and two-loop order. At one-loop order, one finds

$$
\begin{aligned}
& A\left(\ell_{3}, k\right)=2 g\left[-\left(\ell_{3}^{2}+t_{2}\right) \Xi\left(\ell_{3}, k\right)+\ell_{3} \mathcal{B}_{1}(k)\right]+O\left(g^{2}\right) \\
& B\left(\ell_{3}, k\right)=1+2 M g\left[\ell_{3} \Xi\left(\ell_{3}, k\right)-\mathcal{B}_{1}(k)\right]+O\left(g^{2}\right) .
\end{aligned}
$$

The two-loop order contributions are

$$
\begin{aligned}
A_{2}\left(\ell_{3}, k\right)= & 4 M t_{2} \mathcal{B}_{1}(k) \Xi\left(\ell_{3}, k\right), \\
B_{2}\left(\ell_{3}, k\right)= & 2\left[\left(\left(M^{2}-t_{1}\right) \ell_{3}^{2}-t_{1} t_{2}\right) \Xi^{2}\left(\ell_{3}, k\right)+2 \ell_{3}\left(t_{1}-M^{2}\right) \mathcal{B}_{1}(k) \Xi\left(\ell_{3}, k\right)\right. \\
& \left.+\left(M^{2}-t_{1}\right) \mathcal{B}_{1}^{2}(k)\right] .
\end{aligned}
$$

All orders: the massless limit. In the limit $M=0$, the form of the vertex can be guessed to all orders. In terms of the quantity (5.29),

$$
\tau=\sqrt{t_{1} t_{2}+\ell_{3}^{2}\left(t_{1}-M^{2}\right)}
$$

for $M=0$, these expressions can be written as

$$
\begin{aligned}
& A=-\frac{\ell_{3}^{2}+t_{2}}{\tau} \cos \left(g k \mathcal{B}_{1}\right) \sin (2 g \tau \Xi)-\left(\ell_{3} / \sqrt{t_{1}}\right) \sin \left(g k \mathcal{B}_{1}\right) \cos (2 g \tau \Xi) \\
& B=\cos \left(g k \mathcal{B}_{1}\right) \cos (2 g \tau \Xi)-\left(\ell_{3} \sqrt{t_{1}} / \tau\right) \sin \left(g k \mathcal{B}_{1}\right) \sin (2 g \tau \Xi) .
\end{aligned}
$$


All orders. Calculating more terms, and using the experience gained with the scalar function, one guesses that in terms of the quantity (5.29), the exact expressions are

$$
\begin{aligned}
A\left(\ell_{3}, k\right)= & \frac{2 \ell_{3}}{k} \sin \left(k g \mathcal{B}_{1}\right) \cos (2 g \tau \Xi)-\frac{t_{2}+\ell_{3}^{2}}{\tau} \cos \left(k g \mathcal{B}_{1}\right) \sin (2 g \tau \Xi) \\
& +\frac{2 M t_{2}}{k \tau} \sin \left(k g \mathcal{B}_{1}\right) \sin (2 g \tau \Xi), \\
B\left(\ell_{3}, k\right)= & \cos \left(g k \mathcal{B}_{1}\right) \cos (2 g \tau \Xi)+\frac{\ell_{3} k}{2 \tau} \sin \left(g k \mathcal{B}_{1}\right) \sin (2 g \tau \Xi) \\
& -\frac{2 M}{k} \sin \left(g k \mathcal{B}_{1}\right) \cos (2 g \tau \Xi)+\frac{M \ell_{3}}{\tau} \cos \left(g k \mathcal{B}_{1}\right) \sin (2 g \tau \Xi) .
\end{aligned}
$$

In this form, one finds linear combinations of the same trigonometric functions as in expressions (5.30a), (5.30b).

\subsection{Integral equation}

The function $A$ and $B$ are solutions of integral equations of the form (5.34a), (5.34b), but with different boundary conditions, which read

$$
\begin{aligned}
& A\left(\ell_{3}, k\right)=-\frac{2 g}{(2 \pi)^{3}} \int \frac{\mathrm{d}^{3} p\left[M p_{3} A\left(p_{3}, k\right)+\left(p_{3}^{2}+t_{2}\right) B\left(p_{3}, k\right)\right]}{\left(\ell_{3}-p_{3}\right)\left[(p+k / 2)^{2}+M^{2}\right]\left[(p-k / 2)^{2}+M^{2}\right]}, \\
& B\left(\ell_{3}, k\right)=1+\frac{2 g}{(2 \pi)^{3}} \int \frac{\mathrm{d}^{3} p\left[t_{1} A\left(p_{3}, k\right)+M p_{3} B\left(p_{3}, k\right)\right]}{\left(\ell_{3}-p_{3}\right)\left[(p+k / 2)^{2}+M^{2}\right]\left[(p-k / 2)^{2}+M^{2}\right]}
\end{aligned}
$$

Inserting the expressions (8.5a), (8.5b), one obtains the numerators

$$
\begin{aligned}
M p_{3} A\left(p_{3}, k\right)+\left(p_{3}^{2}+t_{2}\right) B\left(p_{3}, k\right)=\frac{2 \tau p_{3}}{k} \sin \left(g k \mathcal{B}_{1}\right) \sin (2 g \tau \Xi) \\
+\left[\left(p_{3}^{2}+t_{2}\right) \cos \left(g k \mathcal{B}_{1}\right)-\frac{2 M t_{2}}{k} \sin \left(g k \mathcal{B}_{1}\right)\right] \cos (2 g \tau \Xi), \\
t_{1} A\left(p_{3}, k\right)+M p_{3} B\left(p_{3}, k\right) \\
=\left[M p_{3} \cos \left(g k \mathcal{B}_{1}\right)+\frac{1}{2} k p_{3} \sin \left(g k \mathcal{B}_{1}\right)\right] \cos (2 g \tau \Xi) \\
\quad+\left[-\tau \cos \left(g k \mathcal{B}_{1}\right)+\frac{2 M \tau}{k} \sin \left(g k \mathcal{B}_{1}\right)\right] \sin (2 g \tau \Xi) .
\end{aligned}
$$

Using the same identities as in the scalar case (for details see B.2), one can check that the functions (8.5a), (8.5b) are indeed the solutions of the integral equations with the proper boundary conditions.

\subsection{From the $J_{3}$ vertex to the $J_{3}$ two-point function}

We follow directly the method of section 6.2. The general relation between the $J_{3}$ vertex and the $J_{3}$ two-point function is

$$
\left\langle\tilde{J}_{3}(k) \tilde{J}_{3}(-k)\right\rangle_{\mathrm{c}}=2 \Omega_{1}(M)-\frac{1}{(2 \pi)^{3}} \int \frac{\mathrm{d}^{3} q \mathcal{N}\left(q_{3}, k\right)}{D m_{2} D p_{2}}
$$


with

$$
\begin{aligned}
\mathcal{N}= & D m_{2}+D p_{2}+\left[4 M q_{3} \cos \left(\chi_{2} / 2\right)+\left(D p_{2}+D m_{2}-4 t_{1}\right) \sin \left(\chi_{2} / 2\right)\right] A\left(q_{3}, k\right) \\
& \left.+\left[4 t_{2}+4 q_{3}^{2}-D p_{2}-D m_{2}\right) \cos \left(\chi_{2} / 2\right)-4 M q_{3} \sin \left(\chi_{2} / 2\right)\right] B\left(q_{3}, k\right),
\end{aligned}
$$

where $\chi_{2} \equiv \chi\left(q_{3}\right)$ (definition (5.15)), $A$ and $B$ are the coefficients of $\mathbf{1}$ and $i \sigma_{3}$ in the vertex function $\tilde{V}_{3}^{1,2}$ (defined in (8.4)) and the relation (5.28) between $(E, F)$ and $(A, B)$ has been used).

We first prove that the relation reduces to

$$
\begin{aligned}
\left\langle\tilde{J}_{3}(k) \tilde{J}_{3}(-k)\right\rangle_{\mathrm{c}}= & 2 \Omega_{1}(M) \\
& -\frac{4}{(2 \pi)^{3}} \int \frac{\mathrm{d}^{3} q\left[M q_{3} A\left(q_{3}, k\right)+\left(t_{2}+q_{3}^{2}\right) B\left(q_{3}, k\right)\right]}{\left[(q+k / 2)^{2}+M^{2}\right]\left[(q-k / 2)^{2}+M^{2}\right]},
\end{aligned}
$$

We use a strategy similar to the one used in section 6.2.

We have to transform the combination

$$
\left(D p_{2}+D m_{2}\right)\left[1+\sin \left(\chi_{2} / 2\right) A\left(q_{3}, k\right)-\cos \left(\chi_{2} / 2\right) B\left(q_{3}, k\right)\right] .
$$

We use the integral equation (8.6a), (8.6b) both for $A$ and $B$ and obtain

$$
\left(D p_{2}+D m_{2}\right)\left[1-\cos \left(\chi_{2} / 2\right)+\frac{2 g}{(2 \pi)^{3}} \int \frac{\mathrm{d}^{3} p \mathcal{N}^{\prime}\left(p_{3}, k\right)}{\left(q_{3}-p_{3}\right) D p_{1} D m_{1}}\right]
$$

with

$$
\begin{aligned}
\mathcal{N}^{\prime}\left(p_{3}, k\right)= & -\left[2 M p_{3} A\left(p_{3}, k\right)+\left(p_{3}^{2}+t_{2}\right) B\left(p_{3}, k\right)\right] \sin \left(\chi_{2} / 2\right) \\
& \left.-\left[t_{1} A\left(p_{3}, k\right)+M p_{3} B\left(p_{3}, k\right)\right] \cos \left(\chi_{2}\right) / 2\right) .
\end{aligned}
$$

We then integrate over $q$ and find the numerator

$$
\begin{aligned}
& 4\left[M p_{3} A\left(p_{3}, k\right)+\left(p_{3}^{2}+t_{2}\right) B\left(p_{3}, k\right)\right]\left[\cos \left(\chi_{2} / 2\right)-1\right] \\
& \left.-4\left[t_{1} A\left(p_{3}, k\right)+M p_{3} B\left(p_{3}, k\right)\right] \sin \left(\chi_{2}\right) / 2\right) .
\end{aligned}
$$

All $\chi$ dependent terms then cancel and the remaining term is

$$
-4\left[M p_{3} A\left(p_{3}, k\right)+\left(p_{3}^{2}+t_{2}\right) B\left(p_{3}, k\right)\right],
$$

in agreement with expression (8.7).

\subsection{The $J_{3}$ two-point function}

Due to current conservation, the current two-point function has the general form

$$
\left\langle J_{\mu}(k) J_{\nu}(-k)\right\rangle=\left(\delta_{\mu \nu}-\frac{1}{k^{2}} k_{\mu} k_{\nu}\right) J\left(k^{2}\right) .
$$

The current two-point function can be determined by calculating only the $J_{3}$ two-point function since

$$
\left\langle J_{3}(k) J_{3}(-k)\right\rangle=\frac{1}{k^{2}}\left(k^{2}-k_{3}^{2}\right) J\left(k^{2}\right) .
$$


The $J_{3}$ two-point function is also given by

$$
\left\langle J_{3}(x) J_{3}(y)\right\rangle=-2\left\langle R_{1}(x) R_{1}(y)\right\rangle+2\left\langle R_{1}(x) R_{2}(y)\right\rangle,
$$

where we have used the property that all functions are real and

$$
\left\langle R_{1}(x) R_{1}(y)\right\rangle=\left\langle R_{2}(x) R_{2}(y)\right\rangle \text { and }\left\langle R_{1}(x) R_{2}(y)\right\rangle=\left\langle R_{2}(x) R_{1}(y)\right\rangle .
$$

Since $\langle R R\rangle$ is already known, only one function remains to be determined.

Equation (6.4) is modified. We have proved in section 8.4 that after subtraction of the terms that cancel the phase factors, the relation becomes (eq. (8.7))

$$
\begin{aligned}
\left\langle\tilde{J}_{3}(k) \tilde{J}_{3}(-k)\right\rangle_{\mathrm{c}}= & 2 \Omega_{1}(M) \\
& -\frac{4}{(2 \pi)^{3}} \int \frac{\mathrm{d}^{3} q\left[M q_{3} A\left(q_{3}, k\right)+\left(t_{2}+q_{3}^{2}\right) B\left(q_{3}, k\right)\right]}{\left[(q+k / 2)^{2}+M^{2}\right]\left[(q-k / 2)^{2}+M^{2}\right]} .
\end{aligned}
$$

Leading (one-loop) order. It is given by

$$
\begin{aligned}
\left\langle\tilde{J}_{3}(k) \tilde{J}_{3}(-k)\right\rangle_{\mathrm{c}}= & 2 \Omega_{1}(M)-\left(k^{2}-k_{3}^{2}\right) \mathcal{B}_{1}(k) \\
& -\frac{1}{(2 \pi)^{3}} \int \frac{\mathrm{d}^{3} p 4 p_{3}^{2}}{\left[(p+k / 2)^{2}+M^{2}\right]\left[(p-k / 2)^{2}+M^{2}\right]} .
\end{aligned}
$$

The calculation of the remaining integral can be found in appendix F.1. One finds (eq. (F.2))

$$
\begin{aligned}
& \frac{1}{(2 \pi)^{3}} \int \frac{\mathrm{d}^{3} p p_{3}^{2}}{\left[(p+k / 2)^{2}+M^{2}\right]\left[(p-k / 2)^{2}+M^{2}\right]}=\frac{1}{4} \Omega_{1}(M)+\frac{\Lambda}{48 \pi} \\
& -\frac{1}{8}\left(k^{2}+4 M^{2}\right) \mathcal{B}_{1}(k)+\frac{k_{3}^{2}}{k^{2}}\left[\frac{1}{8}\left(k^{2}+4 M^{2}\right) \mathcal{B}_{1}(k)+\frac{1}{4} \Omega_{1}(M)-\frac{\Lambda}{16 \pi}\right] .
\end{aligned}
$$

Therefore,

$$
\left\langle\tilde{J}_{3}(k) \tilde{J}_{3}(-k)\right\rangle_{\mathrm{c}}=-\frac{1}{2}\left(1-\frac{k_{3}^{2}}{k^{2}}\right)\left(\left(k^{2}-4 M^{2}\right) \mathcal{B}_{1}(k)+\frac{M}{2 \pi}\right),
$$

where the divergent additive constant is fixed by current conservation.

Higher-loop calculations. After some algebra, one finds

$$
\begin{aligned}
& \left(k^{2}-k_{3}^{2}\right)\left[2 g M \mathcal{B}_{1}^{2}(k)+\frac{1}{3} g^{2}\left(k^{2}-4 M^{2}\right) \mathcal{B}_{1}^{3}(k)-\frac{2}{3} g^{3} M k^{2} \mathcal{B}_{1}^{4}(k)\right. \\
& -\frac{1}{15} g^{4} k^{2}\left(k^{2}-4 M^{2}\right) \mathcal{B}_{1}^{5}(k)+\frac{4}{45} g^{5} M k^{4} \mathcal{B}_{1}^{6}(k) \\
& \left.+\frac{2}{315} g^{6} k^{4}\left(k^{2}-4 M^{2}\right) \mathcal{B}_{1}^{7}(k)+O\left(g^{7}\right)\right] .
\end{aligned}
$$

The known terms sum in the form

$$
\begin{aligned}
& \left\langle\tilde{J}_{3}(k) \tilde{J}_{3}(-k)\right\rangle=\left(1-\frac{k_{3}^{2}}{k^{2}}\right) \\
& \quad \times\left[M\left(\frac{1}{g}-\frac{1}{4 \pi}\right)-\frac{M}{g} \cos \left(2 g k \mathcal{B}_{1}\right)-\frac{1}{4 g k}\left(k^{2}-4 M^{2}\right) \sin \left(2 g k \mathcal{B}_{1}\right)\right] .
\end{aligned}
$$


The result is consistent with expressions (8.5a), (8.5b). Indeed, inserting the expressions (8.5a), (8.5b) into equation (8.7), one finds in the numerator the combination (we have factorized -4$)$

$$
\begin{aligned}
& \frac{-2 M t_{2}}{k} \sin \left(k g \mathcal{B}_{1}\right) \cos (2 g \tau \Xi)+\frac{2 \tau q_{3}}{k} \sin \left(k g \mathcal{B}_{1}\right) \sin (2 g \tau \Xi) \\
& \quad+\left(t_{2}+q_{3}^{2}\right) \cos \left(g k \mathcal{B}_{1}\right) \cos (2 g \tau \Xi)
\end{aligned}
$$

One then expands for $q_{3} \rightarrow \infty$ and keeps the two leading terms, proportional to $q_{3}^{2}$ and $q_{3}^{0}$. The term proportional to $q_{3}^{2}$ can be integrated using the results (B.6) and (8.10). The integration of the term proportional to $q_{3}^{0}$ relies on $S_{n, n}$ in equation (B.5). One then recovers the expression (8.11).

The large momentum or zero mass limit. In the limit $M \rightarrow 0$, the two-point function reduces to

$$
\left\langle\tilde{J}_{3}(k) \tilde{J}_{3}(-k)\right\rangle=-\left(1-\frac{k_{3}^{2}}{k^{2}}\right) \frac{k}{4 g} \sin (g / 4) .
$$

We note that positivity is only satisfied if $\sin (g / 4) / g$ is positive. This again implies $|g| \leq 4 \pi$.

More generally, one verifies that for $g=4 \pi$, the two-point function vanishes linearly, another confirmation that the field theory does not make sense for $|g|>4 \pi$.

Using eqs. (8.8), (8.9) we have in the conformal limit of $M \rightarrow 0$ also

$$
\left\langle\tilde{J}_{-}(k) \tilde{J}_{+}(-k)\right\rangle=-\left(1-\frac{k_{-} k_{+}}{k^{2}}\right) \frac{k}{4 g} \sin (g / 4) .
$$

The current correlation functions in the $M=0$ conformal phase in eq. (8.12) and (8.13) that are calculated here in the $A_{3}=0$ gauge are in agreement with the results of ref. [15] (see there eqs. (33), (36) and (37)), which were calculated in the light-cone gauge.

\subsection{The $R$ three-point function}

We have already evaluated the three-point function at zero momentum (expression (3.50)). We can now evaluate it with only one zero momentum by differentiating expression (6.6) with respect to the bare mass or, in terms of the physical fermion mass

$$
\begin{aligned}
& \langle\tilde{R}(k) \tilde{R}(-k) \tilde{R}(0)\rangle_{\mathrm{c}}=\frac{1}{1-2 g M \Omega_{2}(M)} \frac{\partial}{\partial M}\langle\tilde{R}(k) \tilde{R}(-k)\rangle \\
& =-\frac{4 M \Omega_{2}}{1-2 g M \Omega_{2}}-\frac{8 M}{g\left(1-2 g M \Omega_{2}\right)} \frac{\tan \left(g k \mathcal{B}_{1}(k)\right) / k}{1-2 M \tan \left(g k \mathcal{B}_{1}(k)\right) / k} \\
& \quad-\frac{k^{2}+4 M^{2}}{g\left(1-2 g M \Omega_{2}\right)} \frac{\left(2 / k^{2}\right) \tan ^{2}\left(g k \mathcal{B}_{1}(k)\right)-4 g M \mathcal{B}_{2}(k)\left[1+\tan ^{2}\left(g k \mathcal{B}_{1}(k)\right)\right]}{\left[1-2 M \tan \left(g k \mathcal{B}_{1}(k)\right) / k\right]^{2}},
\end{aligned}
$$

where

$$
\mathcal{B}_{2}(k)==\int \frac{\mathrm{d}^{3} q}{(2 \pi)^{3}} \frac{1}{\left(q^{2}+M^{2}\right)^{2}\left[(k-q)^{2}+M^{2}\right]} .
$$


The general connected $R R R$ three-point at leading order. We find

$$
\begin{aligned}
\langle\tilde{R}(k) \tilde{R}(p) \tilde{R}(-p-k)\rangle_{\mathrm{c}}= & \int \frac{\mathrm{d}^{3} q}{(2 \pi)^{3}} \frac{4 M\left(M^{2}-3 q^{2}+2 p \cdot q-2 k \cdot q+p \cdot k\right)}{\left(q^{2}+M^{2}\right)\left[(q+k)^{2}+M^{2}\right]\left[(q-p)^{2}+M^{2}\right]} \\
= & \int \frac{\mathrm{d}^{3} q}{(2 \pi)^{3}} \frac{2 M\left(8 M^{2}+p^{2}+k^{2}+(p+k)^{2}\right)}{\left(q^{2}+M^{2}\right)\left[(q+k)^{2}+M^{2}\right]\left[(q-p)^{2}+M^{2}\right]} \\
& -4 M\left[\mathcal{B}_{1}(p+k)+\mathcal{B}_{1}(p)+\mathcal{B}_{1}(k)\right] .
\end{aligned}
$$

We define the scalar three-point vertex as

$$
T(k, p)=\int \frac{\mathrm{d}^{3} q}{(2 \pi)^{3}} \frac{1}{\left(q^{2}+M^{2}\right)\left[(q+k)^{2}+M^{2}\right]\left[(q-p)^{2}+M^{2}\right]} .
$$

In particular,

$$
T(k, 0)=\mathcal{B}_{2}(k) .
$$

Then,

$$
\begin{aligned}
\langle\tilde{R}(k) \tilde{R}(p) \tilde{R}(-p-k)\rangle_{\mathrm{c}}= & 2 M\left[8 M^{2}+p^{2}+k^{2}+(p+k)^{2}\right] T(k, p) \\
& -4 M\left[\mathcal{B}_{1}(p+k)+\mathcal{B}_{1}(p)+\mathcal{B}_{1}(k)\right] .
\end{aligned}
$$

The generalization to all orders of the denominator in (8.14) is straightforward. Setting

$$
\Delta_{R}(k)=1-2 M \tan \left(g k \mathcal{B}_{1}(k)\right) / k,
$$

it reads

$$
\Delta_{R}(k) \Delta_{R}(p) \Delta_{R}(p+k) .
$$

However, the generalization to all orders of the function $\langle\tilde{R}(k) \tilde{R}(p) \tilde{R}(-p-k)\rangle_{\mathrm{c}}$ is not straightforward and requires more complicate calculations.

\section{Adding a deformation to the Chern-Simons fermion action}

We now introduce a scalar field $\sigma(x)$ and add two new terms to the action (3.7) of the form

$$
\mathcal{S}_{\sigma}=\int \mathrm{d}^{3} x\left[-\sigma(x) \bar{\psi}(x) \cdot \psi(x)+\frac{N}{3 g_{\sigma}} \sigma^{3}(x)-N \mathcal{R} \sigma(x)\right],
$$

where the new parameters $g_{\sigma}$ and $\mathcal{R}$ are fixed when $N \rightarrow \infty$.

The extra $\sigma(x)$ terms in eq. (9.1) are analogous to the triple-trace deformation $\lambda_{6} \phi(x)^{6}$ added to the Chern-Simons boson theory [16, 22]. The action in eq. (9.1) differs from the action in [16] by the term $\mathcal{R} \sigma(x)$ added here in order to have a proper perturbative meaning to the $\sigma$ term in the action.

From dimensional analysis, one concludes that $\sigma$ has mass dimension 1 and that no other term of dimension 3 or less and odd in $\sigma$ can be added. Moreover, $g_{\sigma}$ is dimensionless and $\mathcal{R}$ has dimension two.

For $\mathcal{R} \neq 0$, in the classical limit $\sigma(x)$ has a non-vanishing expectation value $\sigma$, obtained by varying $\sigma(x)$, which is given by

$$
\sigma^{2}=g_{\sigma} \mathcal{R}
$$


Then, setting

$$
\sigma(x)=\sigma+\varsigma(x)
$$

we note that the expectation value $\sigma$ adds to the bare mass $M_{0}$ and

$$
\mathcal{S}_{\sigma}=\int \mathrm{d}^{3} x\left[-(\sigma+\varsigma(x)) \bar{\psi}(x) \cdot \psi(x)+\frac{N}{3 g_{\sigma}} \varsigma^{3}(x)+\frac{N \sigma}{g_{\sigma}} \varsigma^{2}(x)\right] .
$$

Since the propagator of $\varsigma(x)$ is a constant, the dimension of $\varsigma(x)$, from the viewpoint now of power counting, is $\frac{3}{2}$ in such a way that $\varsigma^{3}(x)$ has dimension $\frac{9}{2}$ and, thus, is irrelevant (non-renormalizable). We show below that, by contrast, in the large $N$ limit the situation is different.

\subsection{Large $N$ limit: gap equation and free energy}

We note that the reflection symmetry $g \rightarrow-g, M_{0} \rightarrow-M_{0}$ is now extended if $g_{\sigma} \rightarrow-g_{\sigma}$ and $\mathcal{R} \rightarrow-\mathcal{R}$ and then the saddle point value $\sigma \rightarrow-\sigma$ and also $M \rightarrow-M$. From now on we thus again choose $M \geq 0$.

First, we perform the transformations of section 3.4 in the addition (9.1). In particular,

$$
\int \mathrm{d}^{3} x \sigma(x) \bar{\psi}(x) \cdot \psi(x) \mapsto \int \mathrm{d}^{2} x \mathrm{~d} t \sigma(t, x)\left[\rho_{1}(t, t, x)+\rho_{2}(t, t, x)\right] .
$$

By varying $\sigma$, we now obtain the additional saddle point equation

$$
\begin{aligned}
\sigma^{2} & =g_{\sigma} \mathcal{R}+g_{\sigma}\left[\rho_{1}(0)+\rho_{2}(0)\right] \\
& =g_{\sigma} \mathcal{R}+g_{\sigma} \int \mathrm{d} \omega\left[\tilde{\rho}_{1}(\omega)+\tilde{\rho}_{2}(\omega)\right]=g_{\sigma}(\mathcal{R}+\langle R\rangle) .
\end{aligned}
$$

In the other saddle point equations, the bare mass $M_{0}$ is simply replaced by $M_{0}+\sigma$. The gap equation (7.1) is thus replaced by

$$
M_{0}+\sigma=M+g \Omega_{1}(M)
$$

and $\sigma$ is solution of (using eq. (3.46))

$$
\begin{aligned}
\sigma^{2} & =g_{\sigma}(\mathcal{R}+\langle R\rangle)=g_{\sigma}\left[\mathcal{R}+2 M \Omega_{1}(M)+g \Omega_{1}^{2}(M)\right], \\
& =g_{\sigma}\left[\mathcal{R}+M \frac{\Lambda-M}{2 \pi}+g\left(\frac{\Lambda-M}{4 \pi}\right)^{2}\right] .
\end{aligned}
$$

Eliminating $\sigma$ between the two equations, we transform the gap equation into

$$
\begin{aligned}
g_{\sigma} & {\left[\mathcal{R}+2 M \Omega_{1}(M)+g \Omega_{1}^{2}(M)\right] } \\
& =M_{0}^{2}-2 M_{0}\left[M+g \Omega_{1}(M)\right]+\left[M+g \Omega_{1}(M)\right]^{2}
\end{aligned}
$$

or, more explicitly,

$$
\begin{aligned}
& {\left[1-\frac{\left(g-g_{\sigma}\right)}{2 \pi}\left(1-\frac{g}{8 \pi}\right)\right] M^{2}+\left(1-\frac{g}{4 \pi}\right)\left[\frac{\left(g-g_{\sigma}\right)}{2 \pi} \Lambda-2 M_{0}\right] M} \\
& \quad-g_{\sigma} \mathcal{R}+M_{0}^{2}-\frac{g}{2 \pi} M_{0} \Lambda+g\left(g-g_{\sigma}\right) \frac{\Lambda^{2}}{16 \pi^{2}}=0,
\end{aligned}
$$

an equation quadratic in $M$. 
The effective $\sigma$ potential. After taking into account the $\lambda$ and $\rho$ saddle point equations, the action density for constant $\sigma$ fields normalized as in eq. (3.35) becomes

$$
V_{\text {eff. }}(\sigma)=-W\left(M\left(M_{0}, \sigma\right)\right)-\mathcal{R} \sigma+\frac{\sigma^{3}}{3 g_{\sigma}}
$$

where $W$ is given by eq. (3.36) and $M\left(M_{0}, \sigma\right)$ is solution of eq. (9.4). Since

$$
\frac{\partial}{\partial \sigma} W\left(M\left(M_{0}, \sigma\right)\right)=\frac{\partial}{\partial M_{0}} W\left(M\left(M_{0}, \sigma\right)\right)=\langle R\rangle,
$$

the expression for $\sigma$ in eq. (9.5) is then obtained from

$$
\frac{\partial}{\partial \sigma} V_{\text {eff. }}(\sigma)=-\langle R\rangle-\mathcal{R}+\frac{\sigma^{2}}{g_{\sigma}}=0 .
$$

Furthermore, by calculating the successive derivatives of $V_{\text {eff. }}$ at the saddle point, one obtains the vertex (or 1PI) functions at zero momentum.

Divergences and counter-terms. We now assume that $g$ and $g_{\sigma}$ need not to be renormalized, which we have checked to some extent. Then the cancellation of divergences in the coefficient of $M$ implies that $M_{0}$ has the form

$$
M_{0}=M_{c}+m
$$

where $m$ is finite and

$$
M_{c}=\left(g-g_{\sigma}\right) \frac{\Lambda}{4 \pi} .
$$

Similarly, the cancellation of divergences in the constant term yields the condition

$$
\mathcal{R}=\mathcal{R}_{c}-\frac{m \Lambda}{2 \pi}+\frac{\eta}{4 \pi} m^{2},
$$

where $\eta$ is a constant parameter and

$$
\mathcal{R}_{c}=\left(g_{\sigma}-g\right) \frac{\Lambda^{2}}{16 \pi^{2}}
$$

The gap equation then reads

$$
\left[1-\frac{\left(g-g_{\sigma}\right)}{2 \pi}\left(1-\frac{g}{8 \pi}\right)\right] M^{2}-2\left(1-\frac{g}{4 \pi}\right) m M+m^{2}\left(1-\eta \frac{g_{\sigma}}{4 \pi}\right)=0 .
$$

Note also that the relation (9.4) becomes

$$
\sigma=\frac{\Lambda}{4 \pi} g_{\sigma}-m+M\left(1-\frac{g}{4 \pi}\right)
$$

and, therefore,

$$
\frac{2 \sigma}{g_{\sigma}}=\frac{\Lambda}{2 \pi}-\frac{2 m}{g_{\sigma}}+\frac{2 M}{g_{\sigma}}\left(1-\frac{g}{4 \pi}\right)
$$


Critical limit. If $m=0$ or $\eta=4 \pi / g_{\sigma}$ the gap equation has the solution $M=0$, which corresponds to massless fermions. For $m \neq 0$ and $\eta=4 \pi / g_{\sigma}$, the gap equation has then also a non-zero solution

$$
M=m \frac{2(1-g / 4 \pi)}{1-\left(g-g_{\sigma}\right)(1-g / 8 \pi) / 2 \pi} .
$$

Since the solution can also be positive, checking which is the leading solution requires calculating the corresponding free energy.

Demanding that the coefficient of $M$ also vanishes yields either $g= \pm 4 \pi$, which here also is a singular point, or $m=0$. Then, both solutions of the gap equation coalesce and $M=0$.

Finally, the gap equation is satisfied for any value of $M \geq 0$ if the coefficient of $M^{2}$ also vanishes, that is, for

$$
\left(\frac{g-g_{\sigma}}{2 \pi}\right)\left(1-\frac{g}{8 \pi}\right)=1 \Leftrightarrow g_{\sigma}=-\frac{(4 \pi-g)^{2}}{8 \pi-g} .
$$

We examine this special situation in section 9.5.

For $M<0$, the analysis is the same; one has just to change $g \rightarrow-g, g_{\sigma} \rightarrow-g_{\sigma}$.

\subsection{Connected scalar two-point functions at zero momentum}

Since $R=\bar{\psi} \psi$ and $\sigma$ are both scalar $\mathrm{U}(N)$-invariant fields, they are coupled. To calculate the two-point functions at zero momentum, we have to differentiate the general saddle point equations twice with respect to $M_{0}$ at $\mathcal{R}$ fixed, with respect to $M_{0}$ and $\mathcal{R}$ and, finally, twice with respect to $\mathcal{R}$.

The $R$ two-point function. The two-point function is given by

$$
\langle\tilde{R}(0) \tilde{R}(0)\rangle_{\mathrm{c}}=\frac{\partial\langle R\rangle}{\partial M_{0}}=\frac{2 \sigma}{g_{\sigma}} \frac{\partial \sigma}{\partial M_{0}},
$$

where eq. (9.5) has been used.

Differentiating eq. (9.4) with respect to $M_{0}$ and combining it with eq. (9.19) to eliminate $\partial \sigma / \partial M_{0}$, we obtain

$$
\left(1-\frac{g}{4 \pi}\right) \frac{\partial M}{\partial M_{0}}=1+\frac{g_{\sigma}}{2 \sigma} \frac{\partial\langle R\rangle}{\partial M_{0}}=1+\frac{g_{\sigma}}{2 \sigma} \frac{\partial\langle R\rangle}{\partial M} \frac{\partial M}{\partial M_{0}} .
$$

We infer

$$
\frac{\partial M}{\partial M_{0}}=\frac{2 \sigma}{g_{\sigma}} \frac{1}{(1-g / 4 \pi)} \mathcal{D}^{-1}(0)
$$

with

$$
\mathcal{D}(k=0)=\frac{2 \sigma}{g_{\sigma}}-\frac{1}{1-g / 4 \pi} \frac{\partial\langle R\rangle}{\partial M} .
$$

We note that

$$
\frac{1}{1-g / 4 \pi} \frac{\partial\langle R\rangle}{\partial M}=\langle\tilde{R}(0) \tilde{R}(0)\rangle_{\mathrm{c}, 0},
$$


where $\langle\tilde{R}(0) \tilde{R}(0)\rangle_{\mathrm{c}, 0} 0$ is given by the expression (3.49). Therefore,

$$
\mathcal{D}(0)=\frac{2 \sigma}{g_{\sigma}}-\langle\tilde{R}(0) \tilde{R}(0)\rangle_{\mathrm{c}, 0}
$$

It follows

$$
\begin{aligned}
\langle\tilde{R}(0) \tilde{R}(0)\rangle_{\mathrm{c}} & =\frac{\partial\langle R\rangle}{\partial M_{0}}=\frac{\partial\langle R\rangle}{\partial M} \frac{\partial M}{\partial M_{0}} \\
& =\frac{2 \sigma}{g_{\sigma}} \frac{1}{(1-g / 4 \pi)} \frac{\partial\langle R\rangle}{\partial M} \mathcal{D}^{-1}(0) \\
& =\frac{2 \sigma}{g_{\sigma}}\langle\tilde{R}(0) \tilde{R}(0)\rangle_{\mathrm{c}, 0} \mathcal{D}^{-1}(0)=-\frac{2 \sigma}{g_{\sigma}}+\left(\frac{2 \sigma}{g_{\sigma}}\right)^{2} \mathcal{D}^{-1}(0) .
\end{aligned}
$$

More explicitly,

$$
\begin{aligned}
\mathcal{D}(0) & =\frac{\Lambda}{2 \pi}-2 \frac{m}{g_{\sigma}}+2 \frac{M}{g_{\sigma}}\left(1-\frac{g}{4 \pi}\right)-\frac{1}{2 \pi}\left[\Lambda-2 M \frac{(1-g / 8 \pi)}{(1-g / 4 \pi)}\right] \\
& =-\frac{2 m}{g_{\sigma}}+2 M\left[\frac{1}{g_{\sigma}}\left(1-\frac{g}{4 \pi}\right)+\frac{1}{2 \pi} \frac{(1-g / 8 \pi)}{(1-g / 4 \pi)}\right] .
\end{aligned}
$$

Then, for $m=0$, the equation $\mathcal{D}=0$ directly implies the relation (9.18). This result is consistent with the existence of a massless pole of the $R$ two-point function when the relation (9.18) is satisfied but the example of the point $g=4 \pi$ shows that we still have to verify that the coefficient of $k^{2}$ in the inverse of the $\tilde{R}(k)$ two-point function does not vanish.

\section{The $\langle R \varsigma\rangle$ two-point function.}

$$
\langle\tilde{\varsigma}(0) \tilde{R}(0)\rangle_{\mathrm{c}}=\frac{\partial \sigma}{\partial M_{0}} .
$$

Then, from (9.20), (9.21), (9.22),

$$
\frac{\partial \sigma}{\partial M_{0}}=-1+\left(1-\frac{g}{4 \pi}\right) \frac{\partial M}{\partial M_{0}}=g_{\sigma} \mathcal{D}^{-1}(0) \frac{\partial\langle R\rangle}{\partial M} .
$$

Thus,

$$
\langle\tilde{\varsigma}(0) \tilde{R}(0)\rangle_{\mathrm{c}}=\frac{g_{\sigma}}{2 \sigma}\langle\tilde{R}(0) \tilde{R}(0)\rangle_{\mathrm{c}} .
$$

This relation will be seen to be a direct consequence of the relation (9.35).

The $\varsigma$ vertex functions at zero momentum. The $\varsigma$ two-point vertex function at zeromomentum can be directly calculated by differentiating expression (9.10) with respect to $\sigma$.

First from eq. (9.4), one infers

$$
\frac{\partial M}{\partial \sigma}=\frac{1}{1-g / 4 \pi} .
$$

Then, from

$$
\frac{\partial^{2} V_{\mathrm{eff}}(\sigma)}{(\partial \sigma)^{2}}=\frac{2 \sigma}{g_{\sigma}}-\frac{\partial\langle R\rangle}{\partial \sigma}=\frac{2 \sigma}{g_{\sigma}}-\frac{\Lambda}{2 \pi}+\frac{M}{\pi} \frac{1-g / 8 \pi}{1-g / 4 \pi}
$$


and eq. (9.17), one derives

$$
\langle\tilde{\varsigma}(0) \tilde{\varsigma}(0)\rangle_{1 \mathrm{PI}}=\frac{\partial^{2} V_{\mathrm{eff}}(\sigma)}{(\partial \sigma)^{2}}=\frac{2 \sigma}{g_{\sigma}}-\frac{\partial\langle R\rangle}{\partial \sigma}=\mathcal{D}(0) .
$$

Similarly, the third derivative yields the three-point function:

$$
\langle\tilde{\varsigma}(0) \tilde{\varsigma}(0) \tilde{\varsigma}(0)\rangle_{1 \mathrm{PI}}=\frac{\partial^{3} V_{\mathrm{eff}}(\sigma)}{(\partial \sigma)^{3}}=\frac{2}{g_{\sigma}}+\frac{1}{\pi} \frac{1-g / 8 \pi}{(1-g / 4 \pi)^{2}} .
$$

Finally, all other $n$-point functions with $n>3$ vanish at zero momentum.

\subsection{The generic $R$ two-point function}

Expanding around the saddle point at second order in the fields,

$$
\sigma(x)=\sigma+\varsigma(x), \quad \rho_{\alpha}\left(t, t^{\prime}, x\right)=\rho_{\alpha}\left(t-t^{\prime}\right)+\varrho_{\alpha}\left(t, t^{\prime}, x\right),
$$

we find that the modified action generates the two additional quadratic terms

$$
N \int \mathrm{d}^{2} x \mathrm{~d} t\left[-\varsigma(x)\left(\varrho_{1}(t, t, x)+\varrho_{2}(t, t, x)\right)+\frac{\sigma}{g_{\sigma}} \varsigma^{2}(x)\right] .
$$

To calculate the $R$ correlation function, we can integrate over $\varsigma$, which amounts to replacing $\varsigma$ by solution of the field equation obtained by differentiating with respect $\varsigma(x)$. One finds the contribution

$$
-\frac{N g_{\sigma}}{4 \sigma} \int \mathrm{d}^{2} x \mathrm{~d} t\left[\varrho_{1}(t, t, x)+\varrho_{2}(t, t, x)\right]^{2},
$$

which adds to the quadratic term in $\varrho$,

$$
\frac{1}{2} g N \int \mathrm{d}^{2} x \mathrm{~d} t \mathrm{~d} t^{\prime} \operatorname{sgn}\left(t^{\prime}-t\right) \rho_{1}\left(t^{\prime}, t, x\right) \rho_{2}\left(t, t^{\prime}, x\right) .
$$

An expansion in powers of $g_{\sigma}$ then leads to

$$
\langle\tilde{R}(k) \tilde{R}(-k)\rangle_{\mathrm{c}}=\frac{\langle\tilde{R}(k) \tilde{R}(-k)\rangle_{\mathrm{c}, 0}}{1-\left(g_{\sigma} / 2 \sigma\right)\langle\tilde{R}(k) \tilde{R}(-k)\rangle_{\mathrm{c}, 0}},
$$

where $\langle\tilde{R}(k) \tilde{R}(-k)\rangle_{\mathrm{c}, 0}$ is the connected two-point function (6.6).

It is convenient to rewrite the expression as

$$
\langle\tilde{R}(k) \tilde{R}(-k)\rangle_{\mathrm{c}}=-\frac{2 \sigma}{g_{\sigma}}+\frac{4 \sigma^{2} / g_{\sigma}^{2}}{2 \sigma / g_{\sigma}-\langle\tilde{R}(k) \tilde{R}(-k)\rangle_{\mathrm{c}, 0}} .
$$

The poles of the two-point function are thus solution of

$$
\mathcal{D}(k) \equiv \frac{2 \sigma}{g_{\sigma}}-2 \Omega_{1}(M)+\frac{k^{2}+4 M^{2}}{k g} \frac{\tan \left(g k \mathcal{B}_{1}(k)\right)}{1-2 M \tan \left(g k \mathcal{B}_{1}(k)\right) / k}=0 .
$$

Using eq. (9.16), we note that

$$
\begin{aligned}
\Omega_{1}(M)-\frac{\sigma}{g_{\sigma}} & =\frac{1}{4 \pi}(\Lambda-M)-\frac{\Lambda}{4 \pi}-\frac{1}{g_{\sigma}}\left[-m+M\left(1-\frac{g}{4 \pi}\right)\right] \\
& =\frac{m}{g_{\sigma}}-\frac{M}{g_{\sigma}}\left(1-\frac{g-g_{\sigma}}{4 \pi}\right) .
\end{aligned}
$$

The denominator of the expression (9.27) is thus a finite quantity, as it should. Moreover, we see that the $R$ two-point function now requires an additive but also a multiplicative renormalization $\sigma / g_{\sigma}$ as eq. (9.27) explicitly shows. 
The $\varsigma$ two-point function. To calculate the $\varsigma$ two-point function, it is convenient to first integrate over $\varrho$ at $R$ fixed. Since we have determined the $R$ two-point function, we know the result of the integral. We introduce the notation

$$
R_{\mathrm{S}}(x)=R(x)-\langle R\rangle .
$$

The effective quadratic action then reads

$$
\begin{aligned}
\frac{1}{N} \mathcal{S}_{\text {eff. }}= & \frac{1}{2} \int \mathrm{d}^{3} x \mathrm{~d}^{3} y R_{\mathrm{s}}(x) R_{\mathrm{s}}(y)\langle R(x) R(y)\rangle_{\mathrm{c}, 0}^{-1} \\
& +\int \mathrm{d}^{3} x\left[-\varsigma(x) R_{\mathrm{s}}(x)+\frac{\sigma}{g_{\sigma}} \varsigma^{2}(x)\right],
\end{aligned}
$$

where in $\langle R(x) R(y)\rangle_{\mathrm{c}, 0}^{-1}$ the inverse has to be understood in the sense of kernels.

Note that if we integrate over $\varsigma$, we recover the inverse connected $R$ two-point function in the form

$$
\langle R(k) R(-k)\rangle_{\mathrm{c}}^{-1}=\langle R(k) R(-k)\rangle_{\mathrm{c}, 0}^{-1}-\frac{g_{\sigma}}{2 \sigma},
$$

which is equivalent to eq. (9.27). However, we can now instead integrate over $R_{\mathrm{s}}$ and find

$$
\frac{1}{N} \mathcal{S}_{\text {eff. }}=-\frac{1}{4} \int \mathrm{d}^{3} x \mathrm{~d}^{3} y \varsigma(x) \varsigma(y)\langle R(x) R(y)\rangle_{\mathrm{c}, 0}+\frac{\sigma}{g_{\sigma}} \int \mathrm{d}^{3} x \varsigma^{2}(x) .
$$

Thus,

$$
\langle\tilde{\varsigma}(k) \tilde{\varsigma}(-k)\rangle=\frac{1}{2 \sigma / g_{\sigma}-\langle\tilde{R}(k) \tilde{R}(-k)\rangle_{\mathrm{c}, 0}} \equiv \mathcal{D}^{-1}(k) .
$$

with (eq. (9.28))

$$
\mathcal{D}(k)=-\frac{2 m}{g_{\sigma}}+\frac{2 M}{g_{\sigma}}\left(1-\frac{g-g_{\sigma}}{4 \pi}\right)+\frac{k^{2}+4 M^{2}}{k g} \frac{\tan \left(g k \mathcal{B}_{1}(k)\right)}{1-2 M \tan \left(g k \mathcal{B}_{1}(k)\right) / k} .
$$

We see that this correlation function is finite in the infinite cut-off limit. We show in section A.2 that the function $\mathcal{D}$ has a zero for $k$ pure imaginary and $|k|<2 M$ if the two conditions are satisfied:

$$
-\frac{M}{4 \pi-g} \leq-\frac{m}{g_{\sigma}}+\frac{M}{g_{\sigma}}\left(1-\frac{g-g_{\sigma}}{4 \pi}\right)<0
$$

the first condition, equivalent to $\mathcal{D}(0) \geq 0$, being necessary for the theory to be physical. This zero corresponds to a massive scalar bound state.

Small $k$ expansion. Concentrating on the situation where $\mathcal{D}(0)$ is positive and small and $M \neq 0$, we can expand the denominator for $k$ small. Using

$$
\begin{aligned}
& \frac{2 M}{k} \tan \left(\left(g k B_{1}(k)\right)\right) \\
& \quad=\frac{g}{4 \pi}\left[1+\frac{1}{3}\left(\frac{k}{2 M}\right)^{2}\left(\left(\frac{g}{4 \pi}\right)^{2}-1\right) \sum_{0}^{\infty}\left(\frac{k}{2 M}\right)^{2 n} P_{n}\left((g / 4 \pi)^{2}\right)\right],
\end{aligned}
$$


where

$$
\begin{aligned}
& P_{0}=1, \quad P_{1}\left((g / 4 \pi)^{2}\right)=\frac{1}{5}\left((g / 4 \pi)^{2}-3\right) \\
& P_{2}\left((g / 4 \pi)^{2}\right)=\frac{1}{105}\left[17(g / 4 \pi)^{4}-53(g / 4 \pi)^{2}+45\right], \ldots \text { etc. }
\end{aligned}
$$

We find

$$
\begin{aligned}
\mathcal{D}(k)= & \frac{M(4 \pi-g)-4 \pi m}{2 \pi g_{\sigma}}+\frac{M(8 \pi-g)}{2 \pi(4 \pi-g)}+\frac{M}{6 \pi} \frac{(8 \pi-g)}{(4 \pi-g)}\left(\frac{k}{2 M}\right)^{2} \\
& +\frac{M}{90 \pi} \frac{(4 \pi+g)}{(4 \pi-g)}\left[2\left(\frac{g}{4 \pi}\right)^{2}+5\left(\frac{g}{4 \pi}\right)-6\right]\left(\frac{k}{2 M}\right)^{4}+O\left(k^{6}\right) .
\end{aligned}
$$

We note that the positivity of the coefficient of $k^{2}$ implies $g<4 \pi$. Then, $\mathcal{D}$ has a zero corresponding to a scalar particle with mass given by

$$
M_{S}^{2} \sim 12 M^{2}+\frac{12 M[M(4 \pi-g)-4 \pi m](4 \pi-g)}{(8 \pi-g) g_{\sigma}},
$$

where $M / m=f\left(g, g_{\sigma}, \eta\right)$ is the solution of the gap equation (9.15).

Remark. From the correlation functions, we note that in the large cut-off limit

$$
\varsigma(x) \underset{\Lambda \rightarrow \infty}{\sim} \frac{g_{\sigma}}{2 \sigma} R_{s}(x)=\left[R_{s}(x)\right]_{\mathrm{ren} .},
$$

and thus $\varsigma$ is the renormalized $R_{s}$ field.

\subsection{The critical limit}

In the limit $M \rightarrow 0_{+}$, the expression reduces to

$$
\mathcal{D}(k)=-\frac{2 m}{g_{\sigma}}+k \frac{\tan (g / 8)}{g},
$$

where either $m=0$ or $\eta=4 \pi / g_{\sigma}$. In the latter case, $m / g_{\sigma}$ must be negative. In both cases, for $g>0$ the positivity condition $\tan (g / 8)>0$ implies $g<4 \pi$.

In the case $m=0$, the connected $\sigma$ two-point function becomes identical to the propagator of a free massless scalar particle:

$$
\langle\tilde{\varsigma}(k) \tilde{\varsigma}(-k)\rangle=\frac{g}{\tan (g / 8)} \frac{1}{k} .
$$

A special limit. A special limit is $g=g_{\sigma}, m=0$ where all physical masses $M$ and $M_{S}$ vanish but also all bare dimensional parameters,

$$
M_{0}=0, \quad \mathcal{R}=0,
$$




\subsection{Situation with vanishing coefficient of $M^{2}$ in the gap equation}

We are especially interested in the case where the relation (9.18) is satisfied. Then,

$$
\mathcal{D}(k)=\frac{2 m(8 \pi-g)}{(4 \pi-g)^{2}}+\frac{(8 \pi-g)}{24 \pi M(4 \pi-g)} k^{2}+O\left(k^{4}\right) .
$$

The physical parameters correspond to $m>0$ and, since $g_{\sigma}$ then is negative, also implies $m / g_{\sigma}<0$.

When the relation (9.18) is satisfied, quite generally eq. (9.16) leads to

$$
M=\frac{m}{2}\left(\frac{4 \pi}{4 \pi-g}+\eta \frac{(4 \pi-g)}{(8 \pi-g)}\right) .
$$

Inserting the value of $M$ in $\mathcal{D}$, we obtain

$$
\mathcal{D}(k)=\frac{2 m(8 \pi-g)}{(4 \pi-g)^{2}}+\frac{(8 \pi-g)^{2}}{12 \pi m\left[4 \pi(8 \pi-g)+\eta(4 \pi-g)^{2}\right]} k^{2}+O\left(k^{4}\right) .
$$

This expression is physical only if

$$
\left[4 \pi(8 \pi-g)+\eta(4 \pi-g)^{2}\right] \geq 0 .
$$

Then, the mass $M_{S}$ of the scalar $\bar{\psi} \psi$ bound state is given by

$$
M_{S}^{2}=24 \pi m^{2}\left(\frac{4 \pi}{(4 \pi-g)^{2}}+\frac{\eta}{8 \pi-g}\right),
$$

where this expression assumes that

$$
\frac{4 \pi}{(4 \pi-g)^{2}}+\frac{\eta}{8 \pi-g} \propto\left|\eta-1 / g_{\sigma}\right| \ll 1 .
$$

If in addition $m=0, M$ is undetermined and the two-point function has the form

$$
\langle\tilde{\varsigma}(k) \tilde{\varsigma}(-k)\rangle \underset{k \rightarrow 0}{\sim} \frac{24 \pi(4 \pi-g)}{(8 \pi-g)} \frac{M}{k^{2}} .
$$

A pole is found for $k=0$, which corresponds to a massless particle provided the residue is positive, which again implies, starting from $g=0$, that $|g|<4 \pi$. Since the mass of the fermions $M$ is arbitrary, this pole may have the interpretation of a dilaton pole. We then expect that all vertex functions vanish at zero momentum. Indeed, one verifies that this condition is satisfied by using the relation (9.18) in the three-point function (9.25), since, as noted, other vertex functions vanish automatically.

The dilaton effective action. At large distance or small momentum limit, for $M \neq 0$ the fermions decouple and one can derive an effective action for the massless dilaton field $\varsigma(x)$ (which is a $\mathrm{U}(N)$ singlet) in the form of a derivative expansion.

It is convenient to normalize the dilaton field,

$$
\varsigma(x)=f_{D}^{-1} D(x),
$$


where $f_{D}$ is defined from eq. (9.41),

$$
\langle\tilde{\varsigma}(k) \tilde{\varsigma}(-k)\rangle \underset{k \rightarrow 0}{\sim} \frac{f_{D}^{2}}{k^{2}}, \quad f_{D}=\sqrt{24 \pi} \sqrt{\frac{1-g / 4 \pi}{2-g / 4 \pi}} \sqrt{M} .
$$

As noted above, all vertex functions vanish at zero momentum and thus the effective action contains no derivative-free terms. The action of the renormalized dilaton field $D(x)$ has then the form

$$
\left.\mathcal{S}(D)=\frac{1}{2} \int \mathrm{d}^{3} x \partial_{\mu} D(x) \partial_{\mu} D(x) F(D(x))+\mathcal{O} \text { (higher order terms in } \partial_{\mu}\right) .
$$

If in the action one keeps only the terms with two derivatives, by a simple change of field coordinates the action can be reduced to a free massless field action, like in the example of the $O(2)$ non-linear $\sigma$ model.

In order to obtain the full interacting dilaton effective action, one has then to calculate the $n$-point vertex (or 1PI) function of $\varsigma(x)$. More on the dilaton effective potential in these three dimensional Chern-Simons boson and fermion theories can be found in [22] and [23].

\subsection{Spontaneously broken scale invariance in boson and fermion theories}

The relation between $3 \mathrm{D} \mathrm{U}(N)$ fermion and boson theories each coupled to Chern-Simons gauge fields, at large $N$, has raised recently much interest $[11,14-16]$. In particular, a precise mapping between the boson and fermion theories was exhibited. Previous published results were obtained within the light-cone gauge and we compared them to our results derived within the temporal gauge in preceding sections. The comparison was done in the case of the massless conformal phase. It is interesting to compare our results also in the case of the massive phase with spontaneously broken scale invariance. The comparison will check the consistency of our results with the light-cone results and at the same time will confirm the boson-fermion duality mapping in the case of the massive phase.

The condition we found in eq. (9.18) for the existence of a massive phase in the fermion case was,

$$
\left(\frac{g-g_{\sigma}}{2 \pi}\right)\left(1-\frac{g}{8 \pi}\right)=1
$$

In the boson theory, the existence of a massive ground state requires [16, 22]

$$
\lambda_{b}^{2}+\frac{\lambda_{6}}{8 \pi^{2}}=4
$$

where $\lambda_{b}$ is the Chern-Simons gauge coupling in the boson theory and $\lambda_{6}$ is the marginal coupling of the boson $\lambda_{6}\left(\phi^{\dagger} \cdot \phi\right)^{3} / 6 N^{2}$ interaction.

It is now interesting to find out whether the boson and fermion conditions in eqs. (9.45) and (9.46) are dual copies when the dual values of the couplings are taken into account. Indeed, using in eq. (9.46) the mapping between the fermion and the boson theories of ref. $[15,16]$, which was derived in the light-cone gauge,

$$
\lambda_{b}=\frac{g}{4 \pi}-1, \quad \lambda_{6}=8 \pi^{2}\left(1-\frac{g}{4 \pi}\right)^{2}\left(3-4 \frac{g}{g_{\sigma}}\right),
$$

one finds that the boson condition in eq. (9.46) and the fermion condition in eq. (9.45) derived in this article in a temporal gauge are identical. 


\section{Summary and conclusions}

In this article, we have presented a detailed analysis of the large $N$ limit of threedimensional $\mathrm{U}(N)$ symmetric field theory with fermion in the fundamental representation coupled to a Chern-Simons gauge field. As mentioned in the introduction, this model rose interest in recent years [1]-[16, 22, 23] since its singlet sector has been conjectured through AdS/CFT correspondence to be related to Vasiliev's [5, 6] higher spin gravity theory on $\mathrm{AdS}_{4}$. Previous analysis of this system were done in the light-cone gauge whereas we worked in the axial $A_{3}=0$ gauge, which is somewhat more difficult to analyse but lacks the peculiarity of the light-cone gauge. We determine the large $N$ properties by using a field integral formalism and the steepest descent method [17]. The saddle point equations, which take here the form of integral equations for non-local fields, determine the ground state phase structure.

The vertex functions and correlators were calculated at leading order in $1 / N$. We derived exact solutions to the integral equations. From the vertex functions, we have inferred two-point correlation functions for scalar and current operators. The calculation were done first in the massive phase away from criticality in the phase in which scale invariance is explicitly broken. Later, the critical massless conformal phase was considered. The results were checked by calculating several orders in perturbation theory. We have compared our results with results obtained in previous works for gauge invariant physical quantities in the conformal massless limit.

In particular, we have thoroughly investigated the conditions for the occurrence of a massive phase in which scale invariance is spontaneously broken and its detailed properties. Indeed, the issue of a possible spontaneously broken massive phase was recently debated in several papers $[16,22,23]$. As described in section 6.3 , we do not find such a massive spontaneously broken phase in our results: the gap equation (7.6) allows the physical fermion mass $M \neq 0$ provided $g= \pm 4 \pi$, however, eq. (6.6) clearly shows that the range of physical values for the coupling is $-4 \pi<g<4 \pi$ leaving the solution $M \neq 0$ outside the physical range. Moreover, spontaneously broken scale symmetry would have required a massless dilaton to appear in the theory when $g= \pm 4 \pi$. This does not happen since the $\langle\tilde{R}(k) \tilde{R}(-k)\rangle_{\mathrm{c}}$ correlator in eq. (6.6) diverges at $g= \pm 4 \pi$ for any value of the momentum $k$ and no massless dilaton pole appears there. Thus, in the critical theory, the conformal invariant massless ground state is the only possible ground state in the Chern-Simons fermion matter system. A massive ground state appears only away from criticality $M_{0}-$ $M_{c}=m \neq 0$ were we have an explicit breaking of scale invariance.

The absence of a massive ground state agrees with the results of ref. [16] calculated in the light-cone gauge. However, in the case of the bosons interacting with Chern-Simons gauge fields a massive spontaneously broken scale invariant phase exists only when a marginal deformation is added of the form $\left(\lambda_{6} / 6 N^{2}\right)\left(\phi^{\dagger} \cdot \phi\right)^{3}$. The massive phase and the dilaton massless pole appears $[16,22]$ in this case when $\lambda^{2}+\lambda_{6} / 8 \pi^{2}=4$. The range of the Chern-Simons coupling $\lambda$ is in this case $-1<\lambda<1$ and the added self interaction

marginal operator allows a massive ground state. In the absence of the Chern-Simons gauge field, this result agrees with ref. [18, 19]. 
As noted in ref. [16], in order for a massive, spontaneously broken scale invariance phase to appear in the fermion case, here too a marginal deformation of the type $\sigma \bar{\psi} \psi+$ $(N / 3 !) \lambda_{6}^{f} \sigma^{3}$ has to be added.

In section 9, we added to the action the above mentioned self-interacting auxiliary field $\sigma(x)$ coupled to the fermions, which effectively induces a marginal deformation to the Chern-Simons fermion action. Indeed, in our $A_{3}=0$ gauge calculations a spontaneously broken massive phase appears and a massless dilaton pole appears in the $\langle\tilde{R}(k) \tilde{R}(-k)\rangle_{\mathrm{c}}$ correlator. We found that the massive phase appears in the spectrum provided the $\sigma$ self interacting coupling $g_{\sigma}$ is related to the Chern-Simons gauge coupling $g$ by $g_{\sigma} / 4 \pi=$ $-(1-g / 4 \pi)^{2} /(2-g / 4 \pi)$ in analogy to the relation between $\lambda_{6}$ and $\lambda$ that ensures a massive ground state in the Chern-Simons boson case [16, 22]. Moreover, in section 9.6 we have shown that the conditions for spontaneous breaking of scale invariance in the boson and fermion theories are dual copies of each other.

\section{Acknowledgments}

We would like to thank O. Aharony, W.A. Bardeen and Ph. Di Francesco for useful remarks, discussions and correspondence on some of the issues covered in this paper. M. Moshe thanks Saclay theoretical institute and CERN theory group for their warm hospitality while different parts of this work were done.

\section{A Some explicit expressions}

We give some technical details about explicit expressions that appear in this work.

\section{A.1 The function $\Theta(\omega)$ and regularization}

To exhibit more clearly the problems arising from the regularization, we calculate the function (3.25) first and Fourier transform only after. Since in momentum space the function is given by a convolution, it becomes a product $(M>0)$ of the form

$$
\frac{\mathrm{e}^{-M|x|}}{|x|} \operatorname{sgn}\left(x_{3}\right) \text {. }
$$

The integration over $p_{1}, p_{2}$ yields $x_{1}=x_{2}=0$. Thus,

$$
\Theta(\omega)=\frac{1}{8 i \pi} \int \mathrm{d} x_{3} \frac{\mathrm{e}^{i \omega x_{3}} \mathrm{e}^{-M\left|x_{3}\right|}}{\left|x_{3}\right|} \operatorname{sgn}\left(x_{3}\right) .
$$

We see that the singularity at $x_{3}=0$ requires a regularization. If we choose a reflection symmetric regularization, $\left|x_{3}\right| \geq \varepsilon>0$, we can divide the integral into $x_{3}>0$ and $x_{3}<0$, change $x_{3}$ into $-x_{3}$ for $x_{3}<0$ and add again the two contributions. The result is

$$
\Theta(\omega)=\frac{1}{8 i \pi} \int_{x_{3}=\varepsilon}^{+\infty} \mathrm{d} x_{3} \frac{\left(\mathrm{e}^{i \omega x_{3}}-\mathrm{e}^{-i \omega x_{3}}\right) \mathrm{e}^{-M x_{3}}}{x_{3}} .
$$


The limit $\varepsilon=0$ can then be taken and the integral calculated. One recovers expression (3.26). By contrast, a non-symmetric short distance cut-off yields a logarithmically divergent limit.

However, we note that in a Feynman parametrization such a symmetrization is automatically achieved when the order of integrations is interchanged and the logarithmic divergence then does not show up:

$$
\begin{aligned}
\Theta(\omega) & =\int \frac{d^{3} p}{(2 \pi)^{3}} \frac{1}{\left(\omega-p_{3}\right)\left(p^{2}+M^{2}\right)} \quad \text { where } \frac{1}{\left(\omega-p_{3}\right)} \quad \text { means PP } \frac{1}{\left(\omega-p_{3}\right)} \\
& =\lim _{\varepsilon \rightarrow 0} \int \frac{d^{3} p}{(2 \pi)^{3}} \int_{0}^{1} \mathrm{~d} x \frac{\omega-p_{3}}{\left[x\left(\omega-p_{3}\right)^{2}+x \varepsilon^{2}+(1-x)\left(p^{2}+M^{2}\right)\right]^{2}} \\
& =\frac{1}{4 \pi} \arctan \left(\frac{\omega}{M}\right) .
\end{aligned}
$$

\section{A.2 The function $\mathcal{D}$ and the bound state problem}

We consider the function (9.30)

$$
\mathcal{D}=-\frac{2 m}{g_{\sigma}}+\frac{2 M}{g_{\sigma}}\left(1-\frac{g-g_{\sigma}}{4 \pi}\right)+\frac{k^{2}+4 M^{2}}{k g} \frac{\tan \left(g k \mathcal{B}_{1}(k)\right)}{1-2 M \tan \left(g k \mathcal{B}_{1}(k)\right) / k}
$$

and assume that the parameters are such that $\mathcal{D}(0)$ is positive. We write it as

$$
\mathcal{D}(k)=\mathcal{D}(0)+[\mathcal{D}(k)-\mathcal{D}(0)]
$$

with

$$
[\mathcal{D}(k)-\mathcal{D}(0)]=\frac{k^{2}+4 M^{2}}{k g} \frac{\tan \left(g k \mathcal{B}_{1}(k)\right)}{1-2 M \tan \left(g k \mathcal{B}_{1}(k)\right) / k}-\frac{2 M}{4 \pi-g} .
$$

We are interested in the real time region below the $\psi \bar{\psi}$ threshold. We thus set

$$
k=2 i M z, \quad 0 \leq z \leq 1, \quad g=4 \pi \lambda, \quad|\lambda|<1 .
$$

and

$$
\begin{aligned}
\phi(z) & =\frac{2 \pi}{M}[\mathcal{D}(k)-\mathcal{D}(0)] \\
& =-\frac{1}{1-\lambda}+\frac{1}{\lambda} \frac{\left(1-z^{2}\right) \tanh \left(\frac{\lambda}{2} \log \left(\frac{z+1}{1-z}\right)\right)}{z-\tanh \left(\frac{\lambda}{2} \log \left(\frac{z+1}{1-z}\right)\right)} \\
& =-\frac{1}{1-\lambda}-\frac{1}{\lambda} \frac{(1+z)^{\lambda}-(1-z)^{\lambda}}{(1+z)^{\lambda-1}-(1-z)^{\lambda-1}} .
\end{aligned}
$$

The function $\phi$ is monotonous decreasing between 0 and 1 , which varies between 0 and $-1 /(1-\lambda)$. Thus, if the condition

$$
\mathcal{D}(0)<\frac{2 M}{4 \pi-g} \Leftrightarrow-\frac{m}{g_{\sigma}}+\frac{M}{g_{\sigma}}\left(1-\frac{g-g_{\sigma}}{4 \pi}\right)<0
$$

is satisfied, one finds a massive scalar bound state. 


\section{B Relevant algebraic identities}

We now describe the algebraic identities that have been systematically used in this work to solve the various integral equations that were encountered.

\section{B.1 Some elementary though useful identities}

In this work, we use systematically identities based on the partial fraction decomposition of rational functions of the form

$$
\prod_{i=1}^{n} \frac{P(z)}{z-\omega_{i}}
$$

where $P$ is a polynomial. If $P$ is of degree smaller than $n$, then

$$
\prod_{i=1}^{n} \frac{P(z)}{z-\omega_{i}}=\sum_{i=1}^{n} \frac{P\left(\omega_{i}\right)}{z-\omega_{i}} \prod_{j \neq i=1}^{n} \frac{1}{\omega_{i}-\omega_{j}} .
$$

In this form, the identity is equivalent to Lagrange interpolation formula.

For higher degrees, additional terms are generated and for the relevant cases one finds

$$
\begin{aligned}
& \prod_{i=1}^{n} \frac{z^{n}}{z-\omega_{i}}=1+\sum_{i=1}^{n} \frac{\omega_{i}^{n}}{z-\omega_{i}} \prod_{j \neq i=1}^{n} \frac{1}{\omega_{i}-\omega_{j}}, \\
& \prod_{i=1}^{n} \frac{z^{n+1}}{z-\omega_{i}}=z+\sum_{i=1}^{n} \omega_{i}+\sum_{i=1}^{n} \frac{\omega_{i}^{n+1}}{z-\omega_{i}} \prod_{j \neq i=1}^{n} \frac{1}{\omega_{i}-\omega_{j}}, \\
& \prod_{i=1}^{n} \frac{z^{n+2}}{z-\omega_{i}}=z^{2}+z \sum_{i=1}^{n} \omega_{i}+\sum_{i \geq j=1}^{n} \omega_{i} \omega_{j}+\sum_{i=1}^{n} \frac{\omega_{i}^{n+2}}{z-\omega_{i}} \prod_{j \neq i=1}^{n} \frac{1}{\omega_{i}-\omega_{j}} .
\end{aligned}
$$

A reformulation of the identities (B.1)-(B.4) where $z \equiv \omega_{n+1}$, is also directly useful. We define

$$
S_{n, m}=\mathfrak{S}_{\left\{\omega_{1}, \omega_{2}, \ldots, \omega_{n+1}\right\}}\left[\omega_{n+1}^{m} \prod_{i=1}^{n} \frac{1}{\omega_{n+1}-\omega_{i}}\right],
$$

where $\mathfrak{S}$ symmetrizes over $\left\{\omega_{1}, \omega_{2}, \ldots, \omega_{n+1}\right\}$. One then finds

$$
S_{n, m}=0 \text { for } m<n, S_{n, n}=\frac{1}{n+1}, S_{n, n+1}=\frac{1}{n+1} \sum_{i=1}^{n+1} \omega_{i} .
$$

Finally, in section 8.5 we need

$$
S_{n, n+2}=\frac{1}{n+1}\left(\sum_{i=1}^{n+1} \omega_{i}^{2}+\sum_{i<j=1}^{n+1} \omega_{i} \omega_{j}\right)
$$

\section{B.2 Some relevant integrals}

We evaluate here a few integrals needed in this work. They involve explicitly the quantity (5.29),

$$
\tau=\frac{1}{2} \sqrt{\left(k^{2}+4 M^{2}\right) t_{2}+p_{3}^{2} k^{2}}=\frac{1}{2} p_{3} k+\frac{t_{2}}{4 p_{3} k}\left(k^{2}+4 M^{2}\right)+O\left(1 / p_{3}^{3}\right) .
$$


From vertex functions to two-point functions. A first set of integrals occurs in the derivation of two-point functions from the corresponding vertex functions.

By expanding in powers of the coupling $g$, the simplest integrals can be inferred from integrals of the form

$$
\frac{1}{(2 \pi)^{3}} \int \frac{\mathrm{d}^{3} p\left(\tau \Xi\left(p_{3}, k\right)\right)^{2 n}}{\left[(p+k / 2)^{2}+M^{2}\right]\left[(p-k / 2)^{2}+M^{2}\right]} .
$$

We then replace the function $\Xi$ by its integral representation,

$$
\Xi\left(p_{3}, k\right)=\frac{1}{(2 \pi)^{3}} \int \frac{\mathrm{d}^{3} q}{\left(p_{3}-q_{3}\right)\left[(q+k / 2)^{2}+M^{2}\right]\left[(q-k / 2)^{2}+M^{2}\right]} .
$$

We symmetrize the integrand over the $(2 n+1)$ integration variables $p, q^{i}$. The numerator has the same degree as the denominator. We use the identities (B.5). Only the term of highest degree in the numerator contributes. Using the value of $S_{n, n}$, we obtain

$$
\frac{1}{2 n+1}\left(k \mathcal{B}_{1} / 2\right)^{2 n+1}
$$

and, thus, the general equation

$$
\frac{1}{(2 \pi)^{3}} \int \frac{\mathrm{d}^{3} p F\left(\tau \Xi\left(p_{3}, k\right)\right)}{\left[(p+k / 2)^{2}+M^{2}\right]\left[(p-k / 2)^{2}+M^{2}\right]}=\int_{0}^{\mathcal{B}_{1}} F(k z / 2) \mathrm{d} z,
$$

for any function $F(z)$ expandable in powers of $z$.

A second type of integrals has the form

$$
\frac{1}{(2 \pi)^{3}} \int \frac{\mathrm{d}^{3} p p_{3}^{2}\left(\tau \Xi\left(p_{3}, k\right)\right)^{2 n}}{\left[(p+k / 2)^{2}+M^{2}\right]\left[(p-k / 2)^{2}+M^{2}\right]} .
$$

We need the two first terms of the expansion for $p_{3}$ large:

$$
p_{3}^{2} \tau^{2 n}=(k / 2)^{2 n} p_{3}^{2 n+2}+\frac{1}{4} n t_{2}\left(k^{2}+4 M^{2}\right)(k / 2)^{2 n-2} p_{3}^{2 n}+O\left(p_{3}^{2 n-2}\right) .
$$

Symmetrizing over all integration momenta and using equations (B.6), (B.5) and noting that the remaining factors in the integrand are even functions, we find

$$
\begin{aligned}
& \left(k \mathcal{B}_{1} / 2\right)^{2 n} \frac{1}{(2 \pi)^{3}} \int \frac{\mathrm{d}^{3} p p_{3}^{2}}{\left[(p+k / 2)^{2}+M^{2}\right]\left[(p-k / 2)^{2}+M^{2}\right]} \\
& +\frac{n}{4(2 n+1)} t_{2}\left(k^{2}+4 M^{2}\right)(k / 2)^{2 n-2} \mathcal{B}_{1}^{2 n+1},
\end{aligned}
$$

and, thus, the general equation

$$
\frac{1}{(2 \pi)^{3}} \int \frac{\mathrm{d}^{3} p p_{3}^{2} F\left(\tau \Xi\left(p_{3}, k\right)\right)}{\left[(p+k / 2)^{2}+M^{2}\right]\left[(p-k / 2)^{2}+M^{2}\right]}=t_{2} \frac{k^{2}+4 M^{2}}{4 k} \int_{0}^{\mathcal{B}_{1}} z F^{\prime}(k z / 2) \mathrm{d} z
$$




$$
+F\left(k \mathcal{B}_{1} / 2\right) \frac{1}{(2 \pi)^{3}} \int \frac{\mathrm{d}^{3} p p_{3}^{2}}{\left[(p+k / 2)^{2}+M^{2}\right]\left[(p-k / 2)^{2}+M^{2}\right]} .
$$

The remaining integral is calculated in section F.1.

A third relevant family of integrals is

$$
\frac{1}{(2 \pi)^{3}} \int \frac{\mathrm{d}^{3} p p_{3} \tau^{2 n} \Xi^{2 n-1}\left(p_{3}, k\right)}{\left[(p+k / 2)^{2}+M^{2}\right]\left[(p-k / 2)^{2}+M^{2}\right]} .
$$

Then, for $p_{3} \rightarrow \infty$,

$$
p_{3} \tau^{2 n}=(k / 2)^{2 n} p_{3}^{2 n+1}+\frac{1}{4} n t_{2}\left(k^{2}+4 M^{2}\right)(k / 2)^{2 n-2} p_{3}^{2 n-1}+O\left(p_{3}^{2 n-3}\right) .
$$

Following the same lines, one obtains

$$
\begin{aligned}
& \frac{1}{(2 \pi)^{3}} \int \frac{\mathrm{d}^{3} p p_{3} \tau F\left(\tau \Xi\left(p_{3}, k\right)\right)}{\left[(p+k / 2)^{2}+M^{2}\right]\left[(p-k / 2)^{2}+M^{2}\right]}=\frac{1}{8} t_{2}\left(k^{2}+4 M^{2}\right) \mathcal{B}_{1}^{2} F(k z / 2) \\
& \quad+F\left(k \mathcal{B}_{1} / 2\right) \frac{1}{(2 \pi)^{3}} \int \frac{\mathrm{d}^{3} p p_{3}^{2}}{\left[(p+k / 2)^{2}+M^{2}\right]\left[(p-k / 2)^{2}+M^{2}\right]} .
\end{aligned}
$$

Integral equations and corresponding integrals. A second set contains integrals relevant for the integral equations. They can be split into two families.

Even functions. A first family has the form

$$
\mathcal{I}_{n}=\frac{1}{(2 \pi)^{3}} \int \frac{\mathrm{d}^{3} p \tau^{2 n} \Xi^{2 n}\left(p_{3}, k\right)}{\left(\ell_{3}-p_{3}\right)\left[(p+k / 2)^{2}+M^{2}\right]\left[(p-k / 2)^{2}+M^{2}\right]} .
$$

We then replace the function $\Xi$ by its integral representation,

$$
\Xi\left(p_{3}, k\right)=\frac{1}{(2 \pi)^{3}} \int \frac{\mathrm{d}^{3} q}{\left(p_{3}-q_{3}\right)\left[(q+k / 2)^{2}+M^{2}\right]\left[(q-k / 2)^{2}+M^{2}\right]} .
$$

We symmetrize the integrand over the $(2 n+1)$ integration variables $p, q^{i}$ and use the identities (B.1). We infer

$$
\mathcal{I}_{n}=\frac{1}{2 n+1} \tau^{2 n}\left(\ell_{3}\right) \Xi^{2 n+1}\left(\ell_{3}, k\right) .
$$

In particular, if $F(z)$ is an even function, expandable at $z=0$, and

$$
\mathcal{I}(F)=\frac{1}{(2 \pi)^{3}} \int \frac{\mathrm{d}^{3} p F\left(\tau \Xi\left(p_{3}, k\right)\right)}{\left(\ell_{3}-p_{3}\right)\left[(p+k / 2)^{2}+M^{2}\right]\left[(p-k / 2)^{2}+M^{2}\right]},
$$

then

$$
\mathcal{I}(F)=\int_{0}^{\Xi} F(\tau z) \mathrm{d} z
$$

A related integral is

$$
\mathcal{J}_{n}=\frac{1}{(2 \pi)^{3}} \int \frac{\mathrm{d}^{3} p p_{3} \tau^{2 n} \Xi^{2 n}\left(p_{3}, k\right)}{\left(\ell_{3}-p_{3}\right)\left[(p+k / 2)^{2}+M^{2}\right]\left[(p-k / 2)^{2}+M^{2}\right]} .
$$


We write it a sum, using $p_{3}=\ell_{3}-\left(\ell_{3}-p_{3}\right)$. The first term yields $\ell_{3} \mathcal{I}_{n}$. We symmetrize the second term and use the identities (B.6). Only the term of highest degree in $p_{3}$ contributes and one finds

$$
-\frac{2}{(2 n+1) k}\left(k \mathcal{B}_{1} / 2\right)^{2 n+1}
$$

For an even function $F$, one then obtains

$$
\mathcal{J}(F)=\ell_{3} \int_{0}^{\Xi} F(\tau z) \mathrm{d} z-\int_{0}^{\mathcal{B}_{1}} F(k z / 2) \mathrm{d} z
$$

A last integral of the family is

$$
\mathcal{K}_{n}=\frac{1}{(2 \pi)^{3}} \int \frac{\mathrm{d}^{3} p p_{3}^{2} \tau^{2 n} \Xi^{2 n}\left(p_{3}, k\right)}{\left(\ell_{3}-p_{3}\right)\left[(p+k / 2)^{2}+M^{2}\right]\left[(p-k / 2)^{2}+M^{2}\right]} .
$$

We substitute this time $p_{3}^{2}=\ell_{3}\left[\ell_{3}-\left(\ell_{3}-p_{3}\right)\right]-p_{3}\left(\ell_{3}-p_{3}\right)$. The first term yields $\ell_{3} \mathcal{J}_{n}$. For the second term, after cancellation of the gauge propagator, we use the identities (B.6). The degree of the denominator is $2 n$ and of the numerator $(2 n+1)$. For $p_{3} \rightarrow \infty$,

$$
p_{3} 2 \tau^{2 n}=(k / 2)^{2 n} p_{3}^{2 n+1}+O\left(p_{3}^{2 n-1}\right) .
$$

Thus, only the term of highest degree contributes. But since it is odd and the remaining integrand is even, it does not contribute. We conclude that if $F$ is an even function,

$$
\mathcal{K}(F)=\ell_{3}^{2} \int_{0}^{\Xi} F(\tau z) \mathrm{d} z-\ell_{3} \int_{0}^{\mathcal{B}_{1}} F(k z / 2) \mathrm{d} z .
$$

Odd functions. We now examine integrals of odd functions. We first need

$$
\mathcal{L}_{n}=\frac{1}{(2 \pi)^{3}} \int \frac{\mathrm{d}^{3} p \tau^{2 n} \Xi^{2 n-1}\left(p_{3}, k\right)}{\left(\ell_{3}-p_{3}\right)\left[(p+k / 2)^{2}+M^{2}\right]\left[(p-k / 2)^{2}+M^{2}\right]} .
$$

Once the function $\Xi$ is replaced by its integral representation, as a function of $p_{3}$, numerator and denominator have the same degree $2 n$. We symmetrize and now use the identities (B.1), (B.2). The result is the sum of two contributions. The first one is

$$
\frac{1}{2 n} \tau^{2 n}\left(\ell_{3}\right) \Xi^{2 n}\left(\ell_{3}, k\right)
$$

The second one comes only from the term of highest degree in $p_{3}$ of $\tau^{2 n}$ :

$$
-\frac{1}{2 n}\left(k \mathcal{B}_{1} / 2\right)^{2 n}
$$

Then, if $F$ is an odd function and

$$
\mathcal{L}(F)=\frac{1}{(2 \pi)^{3}} \int \frac{\mathrm{d}^{3} p \tau F\left(\tau \Xi\left(p_{3}, k\right)\right)}{\left(\ell_{3}-p_{3}\right)\left[(p+k / 2)^{2}+M^{2}\right]\left[(p-k / 2)^{2}+M^{2}\right]},
$$

the result is

$$
\mathcal{L}(F)=\int_{k \mathcal{B}_{1} / 2}^{\tau \Xi} F(z) \mathrm{d} z=\tau \int_{0}^{\Xi} F(\tau z) \mathrm{d} z-\frac{1}{2} k \int_{0}^{\mathcal{B}_{1}} F(k z / 2) \mathrm{d} z .
$$


A related integral is

$$
\mathcal{M}_{n}=\frac{1}{(2 \pi)^{3}} \int \frac{\mathrm{d}^{3} p p_{3} \tau^{2 n} \Xi^{2 n-1}\left(p_{3}, k\right)}{\left(\ell_{3}-p_{3}\right)\left[(p+k / 2)^{2}+M^{2}\right]\left[(p-k / 2)^{2}+M^{2}\right]} .
$$

We substitute $p_{3}=\ell_{3}-\left(\ell_{3}-p_{3}\right)$. The first term yields $\ell_{3} \mathcal{L}_{n}$. The second term leads to a ratio of a polynomial of degree $2 n$ over a polynomial of degree $(2 n-1)$. Using the identities (B.6), we find a sum of odd terms while the integrand is even.

$$
\mathcal{M}(F)=\ell_{3} \mathcal{L}(F)
$$

\section{The $\langle(\bar{\psi} \psi) \psi \bar{\psi}\rangle$ vertex: two-loop calculations}

We calculate the $\langle(\bar{\psi} \psi) \psi \bar{\psi}\rangle$ vertex (or 1PI) function up to two loops for $N$ large.

Notation. To simplify all expressions, we introduce the compact notation

$$
\begin{array}{ll}
D p_{1}=(p+k / 2)^{2}+M^{2}, & D m_{1}=(p-k / 2)^{2}+M^{2}, \\
D p_{2}=(q+k / 2)^{2}+M^{2}, & D m_{2}=(q-k / 2)^{2}+M^{2} .
\end{array}
$$

\section{C.1 Perturbative calculations}

One-loop results. We define

$$
W^{(1,2)}(x ; y, z)=\langle\bar{\psi}(x) \cdot \psi(x) \psi(y) \bar{\psi}(z)\rangle .
$$

We now calculate the corresponding vertex function in the Fourier representation, setting

$$
\tilde{\Gamma}^{(1,2)}(k ; \ell-k / 2, \ell+k / 2)=-E\left(\ell_{3}, k\right)-i \sigma_{3} F\left(\ell_{3}, k\right)
$$

with

$$
E=\sum_{n=0} E_{n} g^{n}, \quad F=\sum_{n=0} F_{n} g^{n}
$$

and the boundary conditions $E_{0}=1, F_{0}=0$.

We use the dressed fermion propagator (3.39) in such a way that only ladder diagrams have to be summed.

The order $g$ reduces to

$$
\begin{aligned}
& E_{1}=-\frac{1}{(2 \pi)^{3}} \int \frac{\mathrm{d}^{3} p 2 M p_{3}}{\left(\ell_{3}-p_{3}\right) D m_{1} D p_{1}} \\
& F_{1}=\frac{1}{(2 \pi)^{3}} \int \frac{\mathrm{d}^{3} p\left(4 t_{1}-D p_{1}-D m_{1}\right)}{2\left(\ell_{3}-p_{3}\right) D m_{1} D p_{1}} .
\end{aligned}
$$

Integrating, one obtains

$$
\begin{aligned}
& E_{1}=-2 M \ell_{3} \Xi\left(k, \ell_{3}\right)+2 M \mathcal{B}_{1}(k) \\
& F_{1}=2 t_{1} \Xi\left(k, \ell_{3}\right)-\frac{1}{2}\left[\Theta\left(\ell_{3}+k_{3} / 2\right)+\Theta\left(\ell_{3}-k_{3} / 2\right)\right] .
\end{aligned}
$$


We have also defined (eq. (5.24))

$$
\tilde{\Gamma}^{(1,2)}\left(k ; \ell-\frac{1}{2} k, \ell+\frac{1}{2} k\right)=-U^{-1}\left(\ell_{3}-\frac{1}{2} k_{3}\right) \tilde{V}^{(1,2)}\left(k ; \ell-\frac{1}{2} k, \ell+\frac{1}{2} k\right) U^{-1}\left(\ell_{3}+\frac{1}{2} k_{3}\right)
$$

with

$$
\tilde{V}^{(1,2)}\left(k ; \ell-\frac{1}{2} k, \ell+\frac{1}{2} k\right)=A\left(\ell_{3}, k\right)+i \sigma_{3} B\left(\ell_{3}, k\right) .
$$

We expand

$$
A=\sum_{n=0} A_{n} g^{n}, \quad B=\sum_{n=0} B_{n} g^{n}
$$

Then $A_{0}=1, B_{0}=0$ and

$$
\begin{aligned}
& A_{1}=-2 M \ell_{3} \Xi\left(k, \ell_{3}\right)+2 M \mathcal{B}_{1}(k) \\
& B_{1}=2 t_{1} \Xi\left(k, \ell_{3}\right),
\end{aligned}
$$

where all quantities are expressed in terms of the fermion physical mass $M$.

The expansion of $\tilde{V}^{(1,2)}$ to order $g$ inserted into expression (5.24) then generates contributions of order $g^{2}$ in the expansion of $\tilde{\Gamma}^{(1,2)}$. They are given by

$$
\begin{aligned}
\delta E_{2}= & t_{1}\left[\Theta\left(\ell_{3}-\frac{1}{2} k_{3}\right)+\Theta\left(\ell_{3}+\frac{1}{2} k_{3}\right)\right] \Xi\left(k, \ell_{3}\right) \\
& -\frac{1}{8}\left[\Theta\left(\ell_{3}-\frac{1}{2} k_{3}\right)+\Theta\left(\ell_{3}+\frac{1}{2} k_{3}\right)\right]^{2}, \\
\delta F_{2}= & -M\left[\mathcal{B}_{1}(k)-\ell_{3} \Xi\left(k, \ell_{3}\right)\right]\left[\Theta\left(\ell_{3}-\frac{1}{2} k_{3}\right)+\Theta\left(\ell_{3}+\frac{1}{2} k_{3}\right)\right] .
\end{aligned}
$$

The vector $\left(A_{2}, B_{2}\right)$ is then obtained by subtracting them from $\left(E_{2}, F_{2}\right)$.

Two-loop calculation. The two-loop contribution corresponds to a sum of diagrams, a vertex correction and two renormalizations of the fermion propagator, which are generated by expanding the one-loop term calculated with the dressed propagator.

Denoting by $q$ the additional integration vector, we obtain the vertex correction by first acting with the product $\mathcal{T}\left(\ell_{3}, q_{3}\right) \mathcal{T}\left(q_{3}, p_{3}\right)$, where $\mathcal{T}$ is the matrix (5.10), on the vector $(1,0)$.

The propagator renormalizations are inferred from the order $g$ in the expansion of $\mathbf{T}$ to order $g$. In the latter contribution, we replace the function $\Theta$ by its integral representation. With the choice of momenta of figure 4 , we substitute

$$
\Theta\left(p_{3}+k_{3} / 2\right)+\Theta\left(p_{3}-k_{3} / 2\right)=\frac{1}{(2 \pi)^{3}} \int \frac{\mathrm{d}^{3} q\left(D m_{2}+D p_{2}\right)}{\left(p_{3}-q_{3}\right) D m_{2} D p_{2}} .
$$

The common denominator of all contributions then is

$$
D=D m_{1} D p_{1} D m_{2} D p_{2}\left(\ell_{3}-q_{3}\right)\left(q_{3}-p_{3}\right) .
$$

After symmetrization over $(p, q)$, the factor $\left(q_{3}-p_{3}\right)$ cancels and the denominators of the integrands reduce to

$$
D^{\prime}=D m_{1} D p_{1} D m_{2} D p_{2}\left(\ell_{3}-p_{3}\right)\left(\ell_{3}-q_{3}\right)
$$


and the numerators become

$$
\begin{aligned}
\mathcal{N}\left(E_{2}\right)= & -\frac{1}{8}\left(D p_{1}+D m_{1}\right)\left(D p_{2}+D m_{2}\right)+\frac{1}{2} t_{1}\left(D p_{1}+D m_{1}+D p_{2}+D m_{2}\right) \\
& +2\left[M^{2} p_{3} q_{3}-t_{1} t_{2}-t_{1}\left[\ell_{3}\left(p_{3}+q_{3}\right)-p_{3} q_{3}\right]\right] \\
\mathcal{N}\left(F_{2}\right)= & \frac{1}{2} M p_{3}\left(D p_{2}+D m_{2}\right)+\frac{1}{2} M q_{3}\left(D p_{1}+D m_{1}\right)+2 M t_{1}\left(2 \ell_{3}-p_{3}-q_{3}\right) .
\end{aligned}
$$

The integration then is simple and yields

$$
\begin{aligned}
E_{2}= & -\frac{1}{8}\left[\Theta\left(\ell_{3}+k_{3} / 2\right)+\Theta\left(\ell_{3}-k_{3} / 2\right)\right]^{2} \\
& +t_{1}\left[\Theta\left(\ell_{3}+k_{3} / 2\right)+\Theta\left(\ell_{3}-k_{3} / 2\right)\right] \Xi\left(\ell_{3}, k\right) \\
& +2\left[\left(M^{2}-t_{1}\right) \ell_{3}^{2}-t_{1} t_{2}\right] \Xi^{2}\left(\ell_{3}, k\right)+2\left(t_{1}+M^{2}\right) \mathcal{B}_{1}^{2}(k), \\
& -4 M^{2} \ell_{3} \mathcal{B}_{1}(k) \Xi\left(\ell_{3}, k\right) \\
F_{2}= & M\left[\Theta\left(\ell_{3}+k_{3} / 2\right)+\Theta\left(\ell_{3}-k_{3} / 2\right)\right]\left[\ell_{3} \Xi\left(\ell_{3}, k\right)-\mathcal{B}_{1}(k)\right] \\
& +4 M t_{1} \Xi\left(\ell_{3}, k\right) \mathcal{B}_{1}(k) .
\end{aligned}
$$

Finally, the correction terms (C.1a), (C.1b) cancel all terms proportional to $\Theta$ functions, justifying to two-loop order the transformation (5.24), and one finds

$$
\begin{aligned}
A_{2}= & 2\left[\left(M^{2}-t_{1}\right) \ell_{3}^{2}-t_{1} t_{2}\right] \Xi^{2}\left(\ell_{3}, k\right)+2\left(t_{1}+M^{2}\right) \mathcal{B}_{1}^{2}(k), \\
& -4 M^{2} \ell_{3} \mathcal{B}_{1}(k) \Xi\left(\ell_{3}, k\right) \\
B_{2}= & 4 M t_{1} \Xi\left(\ell_{3}, k\right) \mathcal{B}_{1}(k) . \\
A_{2}= & -2\left\{\left[t_{1} t_{2}+\ell_{3}^{2}\left(t_{1}-M^{2}\right)\right] \Xi^{2}\left(\ell_{3}, k\right)+2 \ell_{3} M^{2} \mathcal{B}_{1}(k) \Xi\left(\ell_{3}, k\right)\right. \\
& \left.-\left(M^{2}+t_{1}\right) \mathcal{B}_{1}^{2}(k)\right\}, \\
B_{2}= & 4 M t_{1} \mathcal{B}_{1}(k) \Xi\left(\ell_{3}, k\right) . \\
A_{3}= & \frac{4}{3} M\left\{\ell_{3} \Xi^{3}\left[t_{1} t_{2}+\ell_{3}^{2}\left(t_{1}-M^{2}\right)\right]-3 \mathcal{B}_{1} \Xi^{2}\left[t_{1} t_{2}+\ell_{3}^{2}\left(t_{1}-M^{2}\right)\right]\right. \\
& \left.-3 \mathcal{B}_{1}^{2} \Xi \ell_{3}\left(M^{2}+t_{1}\right)+5 t_{1} \mathcal{B}_{1}^{3}\right\}, \\
B_{3}= & \frac{4}{3} t_{1} \Xi\left\{-\left[t_{1} t_{2}+\ell_{3}^{2}\left(t_{1}-M^{2}\right)\right] \Xi^{2}+3 \mathcal{B}_{1}^{2}\left(t_{1}+M^{2}\right)+4 \Xi \mathcal{B}_{1}^{3}\right\}, \\
A_{4}= & \frac{2}{3}\left\{\left[t_{1} t_{2}+\ell_{3}^{2}\left(t_{1}-M^{2}\right)\right]^{2} \Xi^{4}+4 M^{2} \ell_{3}\left[t_{1} t_{2}+\ell_{3}^{2}\left(t_{1}-M^{2}\right)\right] \Xi^{3} \mathcal{B}_{1}\right. \\
& -6 \Xi^{2} \mathcal{B}_{1}^{2}\left(t+M^{2}\right)\left[t_{1} t_{2}+\ell_{3}^{2}\left(t_{1}-M^{2}\right)\right]-4 \ell_{3} M^{2} \Xi \mathcal{B}_{1}^{3}\left(M^{2}+5 t_{1}\right) \\
& \left.+\mathcal{B}_{1}^{4}\left(5 t_{1}^{2}+18 t_{1} M^{2}+M^{4}\right)\right\}, \\
B_{4}= & \frac{8}{3} t_{1} M \mathcal{B}_{1} \Xi\left\{\left[t_{1} t_{2}+\ell_{3}^{2}\left(t_{1}-M^{2}\right)\right] \Xi^{2}+\left(M^{2}+5 t_{1}\right) \mathcal{B}_{1}^{2}\right\}
\end{aligned}
$$

\section{The $J_{3}$ vertex function at two loops}

We calculate now the $J_{3}=i \bar{\psi} \sigma_{3} \psi$ vertex function. The calculation is very similar to the scalar vertex function but the boundary conditions are now $E_{0}=0, F_{0}=1$. 
At one loop the calculation is simple and one finds

$$
\begin{aligned}
& E_{1}=2\left[-\left(\ell_{3}^{2}+t_{2}\right) \Xi\left(\ell_{3}, k\right)+\ell_{3} \mathcal{B}_{1}(k)\right]+\frac{1}{2} g\left[\Theta\left(\ell_{3}-k_{3} / 2\right)+\Theta\left(\ell_{3}+k_{3} / 2\right)\right] \\
& F_{1}=2 M\left[\ell_{3} \Xi\left(\ell_{3}, k\right)-\mathcal{B}_{1}(k)\right] .
\end{aligned}
$$

This suggests introducing the transformation (8.3),

$$
\left\langle J_{3} \psi \bar{\psi}\right\rangle_{1 \mathrm{PI}}=-U^{-1} V_{J_{3}}^{(2)} U^{-1}
$$

with

$$
\tilde{V}_{J_{3}}^{(2)}\left(k ; \ell-\frac{1}{2} k, \ell+\frac{1}{2} k\right)=A\left(\ell_{3}, k\right)+i \sigma_{3} B\left(\ell_{3}, k\right) .
$$

Then, $A_{0}=0, B_{0}=1$ and

$$
\begin{aligned}
& A_{1}=2\left[-\left(\ell_{3}^{2}+t_{2}\right) \Xi\left(\ell_{3}, k\right)+\ell_{3} \mathcal{B}_{1}(k)\right] \\
& B_{1}=2 M\left[\ell_{3} \Xi\left(\ell_{3}, k\right)-\mathcal{B}_{1}(k)\right] .
\end{aligned}
$$

The expansion of expression (8.3) then generates the additional contributions

$$
\begin{aligned}
\delta E_{2}= & M\left[\ell_{3} \Xi\left(\ell_{3}, k\right)-\mathcal{B}_{1}(k)\right]\left[\Theta\left(\ell_{3}-k_{3} / 2\right)+\Theta\left(\ell_{3}+k_{3} / 2\right)\right] \\
\delta F_{2}= & {\left[\left(\ell_{3}^{2}+t_{2}\right) \Xi\left(\ell_{3}, k\right)-\ell_{3} \mathcal{B}_{1}(k)\right]\left[\Theta\left(\ell_{3}-k_{3} / 2\right)+\Theta\left(\ell_{3}+k_{3} / 2\right)\right] } \\
& -\frac{1}{8}\left[\Theta\left(\ell_{3}-k_{3} / 2\right)+\Theta\left(\ell_{3}+k_{3} / 2\right)\right]^{2} .
\end{aligned}
$$

At two-loops, after some algebra, one obtains

$$
\begin{aligned}
E_{2}\left(\ell_{3}, k\right)= & M\left[\ell_{3} \Xi\left(\ell_{3}, k\right)-\mathcal{B}_{1}(k)\right]\left[\Theta\left(\ell_{3}-k_{3} / 2\right)+\Theta\left(\ell_{3}+k_{3} / 2\right)\right] \\
& +4 M t_{2} \ell_{3} \Xi\left(\ell_{3}, k\right) \mathcal{B}_{1}(k) \\
F_{2}\left(\ell_{3}, k\right)= & -\frac{1}{8}\left[\Theta\left(\ell_{3}-k_{3} / 2\right)+\Theta\left(\ell_{3}+k_{3} / 2\right)\right]^{2} \\
& +\left[\Theta\left(\ell_{3}-k_{3} / 2\right)+\Theta\left(\ell_{3}+k_{3} / 2\right)\right]\left[\left(\ell_{3}^{2}+t_{2}\right) \ell_{3} \Xi\left(\ell_{3}, k\right)-\mathcal{B}_{1}(k)\right] \\
& +2\left(\left(M^{2}-t_{1}\right) \ell_{3}^{2}-t_{1} t_{2}\right) \Xi^{2}\left(\ell_{3}, k\right)-4\left(M^{2}-t_{1}\right) \ell_{3} \Xi\left(\ell_{3}, k\right) \mathcal{B}_{1}(k) \\
& +2\left(M^{2}-t_{1}\right) \mathcal{B}_{1}^{2}(k) .
\end{aligned}
$$

Subtracting $\delta E_{2}$ and $\delta F_{2}$, we obtain

$$
\begin{aligned}
A_{2}\left(\ell_{3}, k\right)= & 4 M t_{2} \ell_{3} \Xi\left(\ell_{3}, k\right) \mathcal{B}_{1}(k) \\
B_{2}\left(\ell_{3}, k\right)= & 2\left(\left(M^{2}-t_{1}\right) \ell_{3}^{2}-t_{1} t_{2}\right) \Xi^{2}\left(\ell_{3}, k\right)-4\left(M^{2}-t_{1}\right) \ell_{3} \Xi\left(\ell_{3}, k\right) \mathcal{B}_{1}(k) \\
& +2\left(M^{2}-t_{1}\right) \mathcal{B}_{1}^{2}(k) .
\end{aligned}
$$

\section{E The $R$ two-point function}

First, we give now some details about the perturbative calculations of the $R$ two-point function. 


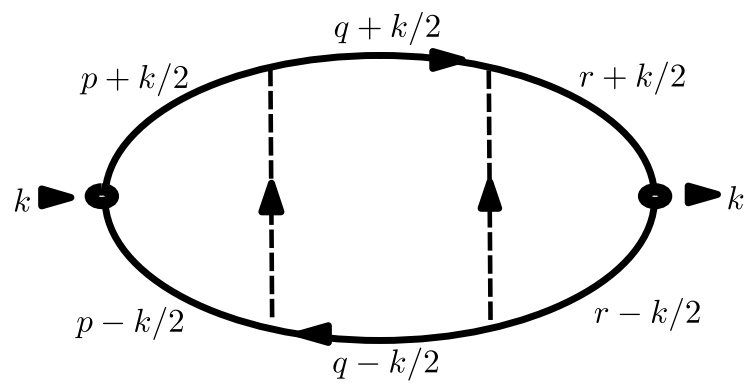

Figure 7. The $\bar{\psi} \psi$ two-point function at two loops. Dotted lines represent gauge fields.

\section{E.1 The $R$ two-point function at three loops}

The first diagram is represented in figure 7 while the other diagrams all correspond to propagator corrections applied to one-loop and two-loop diagrams. To display the different contributions, we complete the compact notation introduced in section $\mathrm{C}$. We define

$$
D p_{3}=(r+k / 2)^{2}+M^{2}, \quad D m_{3}=(r-k / 2)^{2}+M^{2} .
$$

Then, the denominator in the diagram is the product

$$
D p_{1} D m_{1} D p_{2} D m_{2} D p_{3} D m_{3}\left(q_{3}-p_{3}\right)\left(r_{3}-q_{3}\right),
$$

which, up to the factor $\left(q_{3}-p_{3}\right)\left(r_{3}-q_{3}\right)$, is totally symmetric in $(p, q, r)$.

After some algebra, the terms that give vanishing contributions due to rotation symmetry in the $(1,2)$ plane being omitted, the numerator can be written as

$$
\begin{aligned}
& -\frac{1}{4}\left(D p_{1}+D m_{1}\right)\left(D p_{2}+D m_{2}\right)\left(D p_{3}+D m_{3}\right) \\
& +\left(t_{2}+q_{3}^{2}\right)\left(D p_{1}+D m_{1}\right)\left(D p_{3}+D m_{3}\right) \\
& +t_{1}\left(D p_{2}+D m_{2}\right)\left(D p_{1}+D m_{1}+D p_{3}+D m_{3}\right) \\
& -4\left(t_{2}+q_{3}^{2}\right) t_{1}\left(D p_{1}+D m_{1}+D p_{3}+D m_{3}\right) \\
& -4 t_{1}^{2}\left(D p_{2}+D m_{2}\right)-4 M^{2} p_{3} r_{3}\left(D p_{2}+D m_{2}\right) \\
& +4 M^{2} q_{3} r_{3}\left(D p_{1}+D m_{1}\right)+4 M^{2} p_{3} q_{3}\left(D p_{3}+D m_{3}\right) \\
& +16\left(t_{2}+q_{3}^{2}\right) t_{1}^{2}+16 M^{2} t_{1}\left(p_{3} r_{3}-p_{3} q_{3}-q_{3} r_{3}\right) .
\end{aligned}
$$

The identity (B.5) implies that, in the numerator, all quantities symmetric in $(p, q, r)$ give a vanishing contribution. This leads to a notable reduction of the number of terms. Moreover, this implies that one can substitute

$$
\left(D p_{2}+D m_{2}\right)\left(D p_{1}+D m_{1}+D p_{3}+D m_{3}\right) \mapsto-\left(D p_{1}+D m_{1}\right)\left(D p_{3}+D m_{3}\right)
$$

as well as

$$
\begin{aligned}
& 4 M^{2} q_{3} r_{3}\left(D p_{1}+D m_{1}\right)+4 M^{2} p_{3} q_{3}\left(D p_{3}+D m_{3}\right) \mapsto-4 M^{2} p_{3} r_{3}\left(D p_{2}+D m_{2}\right) \\
& -k^{2} t_{1}\left(D p_{1}+D m_{1}+D p_{3}+D m_{3}\right) \mapsto k^{2} t_{1}\left(D p_{2}+D m_{2}\right) .
\end{aligned}
$$


One then obtains

$$
\begin{aligned}
& \left(t_{2}-t_{1}+q_{3}^{2}\right)\left(D p_{1}+D m_{1}\right)\left(D p_{3}+D m_{3}\right) \\
& -4\left(q_{3}^{2}-\frac{1}{4} k_{3}^{2}\right) t_{1}\left(D p_{1}+D m_{1}+D p_{3}+D m_{3}\right) \\
& -4 M^{2}\left(t_{1}+2 p_{3} r_{3}\right)\left(D p_{2}+D m_{2}\right) \\
& +4 t_{1} k^{2} q_{3}^{2}+16 M^{2} t_{1}\left(q_{3}-p_{3}\right)\left(q_{3}-r_{3}\right) .
\end{aligned}
$$

Introducing the integral representation,

$$
\Theta(\omega)=\frac{1}{(2 \pi)^{3}} \int \frac{\mathrm{d}^{3} q}{\left(\omega-q_{3}\right)\left(q^{2}+M^{2}\right)},
$$

one verifies that the first line then yields

$$
-\frac{1}{D p_{2} D m_{2}}\left(q_{3}^{2}-\frac{1}{4} k_{3}^{2}-M^{2}\right) \frac{\chi^{2}\left(q_{3}\right)}{g^{2}} .
$$

The second line can be expressed in the form

$$
-\frac{\left(q_{3}^{2}-\frac{1}{4} k_{3}^{2}\right)\left(k^{2}+4 M^{2}\right) \chi\left(q_{3}\right)}{g D p_{2} D m_{2}}\left[\frac{1}{\left(p_{3}-q_{3}\right) D p_{1} D m_{1}}+\frac{1}{\left(r_{3}-q_{3}\right) D p_{3} D m_{3}}\right] .
$$

Exchanging in the first term above $(r \longleftrightarrow q)$ and in the second $(p \longleftrightarrow q)$, one obtains

$$
\frac{\left(k^{2}+4 M^{2}\right)}{g D p_{1} D m_{1} D p_{3} D m_{3}\left(p_{3}-r_{3}\right)}\left[\left(p_{3}^{2}-\frac{1}{4} k_{3}^{2}\right) \chi\left(p_{3}\right)-\left(r_{3}^{2}-\frac{1}{4} k_{3}^{2}\right) \chi\left(r_{3}\right)\right],
$$

a form useful later.

Using the identity (B.1), one verifies that the third line can be rewritten as

$$
-\frac{M^{2}\left(k^{2}+4 M^{2}+8 p_{3} r_{3}\right)}{g D p_{1} D m_{1} D p_{3} D m_{3}\left(p_{3}-r_{3}\right)}\left(\chi\left(p_{3}\right)-\chi\left(r_{3}\right)\right) .
$$

Finally, the two last terms yield

$$
-\frac{1}{3}\left(k^{2}+12 M^{2}\right)\left(k^{2}+4 M^{2}\right) \mathcal{B}_{1}^{3}(k),
$$

where we have used the identities (B.1), (B.2).

Collecting all contributions, except the last line of expression (E.1), one obtains

$$
\begin{aligned}
& -\frac{1}{D p_{2} D m_{2}}\left(q_{3}^{2}-\frac{1}{4} k_{3}^{2}-M^{2}\right) \frac{\chi^{2}\left(q_{3}\right)}{g^{2}} \\
& -\frac{\left(k^{2}+4 M^{2}\right)}{D p_{1} D m_{1} D p_{3} D m_{3}\left(r_{3}-p_{3}\right) g}\left[\left(p_{3}^{2}-\frac{1}{4} k_{3}^{2}\right) \chi\left(p_{3}\right)-\left(r_{3}^{2}-\frac{1}{4} k_{3}^{2}\right) \chi\left(r_{3}\right)\right] \\
& +\frac{M^{2}\left(k^{2}+4 M^{2}+8 p_{3} r_{3}\right)}{D p_{1} D m_{1} D p_{3} D m_{3}\left(r_{3}-p_{3}\right) g}\left(\chi\left(p_{3}\right)-\chi\left(r_{3}\right)\right) .
\end{aligned}
$$


Propagator renormalizations in the one-loop diagram. Two insertions of the oneloop propagator corrections in the one-loop diagram (first diagram of figure 5), which can be also been inferred by using the dressed propagator and expanding to order $g^{2}$, yield

$$
\frac{1}{D p_{2} D m_{2}}\left(M^{2}+\frac{1}{4} k_{3}^{2}-q_{3}^{2}\right) \frac{\chi^{2}\left(q_{3}\right)}{g^{2}} .
$$

Propagator renormalizations in the two-loop diagram. The sum of all possible insertions of the one-loop propagator correction into the two-loop diagram contribution to $\langle R R\rangle$ (second diagram in figure 5) can be written as the ratio of

$$
\begin{aligned}
& M^{2}\left[\chi\left(r_{3}\right)-\chi\left(p_{3}\right)\right]\left(k^{2}+4 M^{2}+8 p_{3} r_{3}\right) \\
& +\left(k^{2}+4 M^{2}\right)\left[\chi\left(p_{3}\right)\left(p_{3}^{2}-\frac{1}{4} k_{3}^{2}\right)-\chi\left(r_{3}\right)\left(r_{3}^{2}-\frac{1}{4} k_{3}^{2}\right)\right] \\
& +\chi\left(p_{3}\right)\left(\frac{1}{4} k_{3}^{2}+M^{2}-p_{3}^{2}\right)\left(D p_{3}+D m_{3}\right)-\chi\left(r_{3}\right)\left(\frac{1}{4} k_{3}^{2}+M^{2}-r_{3}^{2}\right)\left(D p_{1}+D m_{1}\right)
\end{aligned}
$$

and

$$
g\left(r_{3}-p_{3}\right) D p_{1} D m_{1} D p_{3} D m_{3} .
$$

The expression (E.5a) exactly cancels the expression (E.3c).

The expression (E.5b) then cancels expression (E.3b).

Moreover, the expression (E.5c) can be rewritten as

$$
-\frac{\chi^{2}\left(p_{3}\right)}{g^{2}}\left(\frac{1}{4} k_{3}^{2}+M^{2}-p_{3}^{2}\right)-\frac{\chi^{2}\left(r_{3}\right)}{g^{2}}\left(\frac{1}{4} k_{3}^{2}+M^{2}-r_{3}^{2}\right) .
$$

After an appropriate permutation of variables, it cancels the sum of expressions (E.3a) and (E.4). The final result is

$$
-\frac{1}{3} g^{2}\left(k^{2}+12 M^{2}\right)\left(k^{2}+4 M^{2}\right) \mathcal{B}_{1}^{3}(k),
$$

\section{E.2 The $R$ two-point function at four loops}

Using now the formalism developed in section 5.4, we can calculate the $R$ two-point function at four loops. We set

$$
t_{1}=\frac{1}{2}\left(k_{3}^{2}-k^{2}\right), t_{2}=\frac{1}{2}\left(k^{2}+4 M^{2}\right),
$$

and factorize for each of the four set of integration variables the integral

$$
\frac{1}{(2 \pi)^{3}} \int \frac{\mathrm{d}^{2} v}{\left[(v-k / 2)^{2}+M^{2}\right]\left[(v-k / 2)^{2}+M^{2}\right]} .
$$

The product of the four integrals is the symmetric function of the remaining four integration variables, which we denote by $I(\omega)$.

Denoting $\omega_{1}, \omega_{2}, \omega_{3}, \omega_{4}$ the third components of the four vectors, we obtain an integral of the form

$$
\langle\tilde{R}(k) \tilde{R}(-k)\rangle_{4}=2 g^{3} M t_{2} \int \frac{\mathrm{d} \omega_{1} \mathrm{~d} \omega_{2} \mathrm{~d} \omega_{3} \mathrm{~d} \omega_{4} N(\omega)}{\left(\omega_{4}-\omega_{3}\right)\left(\omega_{3}-\omega_{2}\right)\left(\omega_{2}-\omega_{1}\right)} I(\omega)
$$


with

$$
\begin{aligned}
N(\omega)= & -2 t_{1} t_{2}\left(\omega_{4}-\omega_{3}+\omega_{2}-\omega_{1}\right)+4 t_{2}\left[\left(\omega_{4}-\omega_{3}\right) \omega_{2}^{2}+\omega_{3}^{2}\left(\omega_{2}-\omega_{1}\right)\right] \\
& +8 M^{2}\left(\omega_{1} \omega_{2} \omega_{3}-\omega_{4} \omega_{2} \omega_{1}+\omega_{4} \omega_{3} \omega_{1}-\omega_{4} \omega_{3} \omega_{2}\right) .
\end{aligned}
$$

The integral of the first term vanishes and this restores $O(3)$ symmetry. The second term yields

$$
-\frac{16}{3} M t_{2}^{2} \mathcal{B}_{1}^{4}(k)=-\frac{4}{3} M\left(k^{2}+4 M^{2}\right)^{2} \mathcal{B}_{1}^{4}(k) .
$$

The last term yields

$$
-\frac{8}{3} M^{3}\left(k^{2}+4 M^{2}\right) \mathcal{B}_{1}^{4}(k)
$$

Thus,

$$
\langle\tilde{R}(k) \tilde{R}(-k)\rangle_{4}=-\frac{4}{3} g^{3} M\left(k^{2}+4 M^{2}\right)\left(k^{2}+6 M^{2}\right) \mathcal{B}_{1}^{4}(k) .
$$

Under the assumption that the phase factor cancel, as observed up to three loops and later proved, the successive contributions have been determined up to eight loops.

\section{F Two-point $J_{3}$ calculations}

In this calculation, to keep the notation simple, we assume $M>0$.

\section{F.1 One-loop calculation}

The complete the one-loop calculation of $\left\langle J_{3} J_{3}\right\rangle$, we need

$$
X_{0}=\frac{1}{(2 \pi)^{3}} \int \frac{\mathrm{d}^{3} p p_{3}^{2}}{\left[(p+k / 2)^{2}+M^{2}\right]\left[(p-k / 2)^{2}+M^{2}\right]} .
$$

Then,

$$
\begin{aligned}
& \frac{\partial X_{0}}{\partial M}=-4 M \frac{1}{(2 \pi)^{3}} \int \frac{\mathrm{d}^{3} p p_{3}^{2}}{\left[(p+k / 2)^{2}+M^{2}\right]^{2}\left[(p-k / 2)^{2}+M^{2}\right]} . \\
& \frac{1}{(2 \pi)^{3}} \int \frac{\mathrm{d}^{3} p p_{\mu} p_{\nu}}{\left[(p+k / 2)^{2}+M^{2}\right]^{2}\left[(p-k / 2)^{2}+M^{2}\right]}=A\left(\delta_{\mu \nu}-\frac{k_{\mu} k_{\nu}}{k^{2}}\right)+B \frac{k_{\mu} k_{\nu}}{k^{2}} .
\end{aligned}
$$

Taking the trace, we obtain

$$
2 A+B=\frac{1}{(2 \pi)^{3}} \int \frac{\mathrm{d}^{3} p p^{2}}{\left[(p+k / 2)^{2}+M^{2}\right]^{2}\left[(p-k / 2)^{2}+M^{2}\right]}
$$

Since

$$
p^{2}=\frac{1}{2}\left[(p+k / 2)^{2}+M^{2}\right]+\frac{1}{2}\left[(p-k / 2)^{2}+M^{2}\right]-k^{2} / 4-M^{2},
$$

then,

$$
2 A+B=\frac{1}{2} \mathcal{B}_{1}(k)+\frac{1}{2} \Omega_{2}(M)-\frac{1}{4}\left(k^{2}+4 M^{2}\right) X_{1}
$$

with

$$
X_{1}=\frac{1}{(2 \pi)^{3}} \int \frac{\mathrm{d}^{3} p}{\left[(p+k / 2)^{2}+M^{2}\right]^{2}\left[(p-k / 2)^{2}+M^{2}\right]} .
$$


Moreover,

$$
\frac{\partial \mathcal{B}_{1}}{\partial M}=-4 M \frac{1}{(2 \pi)^{3}} \int \frac{\mathrm{d}^{3} p}{\left[(p+k / 2)^{2}+M^{2}\right]^{2}\left[(p-k / 2)^{2}+M^{2}\right]} .
$$

Thus,

$$
X_{1}=-\frac{1}{4 M} \frac{\partial \mathcal{B}_{1}}{\partial M}
$$

A second relation is obtained by acting with $k_{\nu}$ :

$$
B k^{2}=\frac{1}{(2 \pi)^{3}} \int \frac{\mathrm{d}^{3} p(k p)^{2}}{\left[(p+k / 2)^{2}+M^{2}\right]^{2}\left[(p-k / 2)^{2}+M^{2}\right]} .
$$

Then,

$$
k p=\frac{1}{2}\left[(p+k / 2)^{2}+M^{2}\right]-\frac{1}{2}\left[(p-k / 2)^{2}+M^{2}\right]
$$

and, thus,

$$
B k^{2}=-\frac{1}{2} \frac{1}{(2 \pi)^{3}} \int \frac{\mathrm{d}^{3} p k p}{\left[(p+k / 2)^{2}+M^{2}\right]^{2}}=\frac{1}{4} k^{2} \Omega_{2}(M) .
$$

We conclude

$$
B=\frac{1}{4} \Omega_{2}(M), \quad A=\frac{1}{4} \mathcal{B}_{1}(k)+\frac{1}{8} \Omega_{2}(M)+\frac{1}{32 M}\left(k^{2}+4 M^{2}\right) \frac{\partial \mathcal{B}_{1}}{\partial M} .
$$

Finally,

$$
\begin{aligned}
\frac{\partial X_{0}}{\partial M}= & -4 M\left[A\left(1-k_{3}^{2} / k^{2}\right)+B k_{3}^{2} / k^{2}\right] \\
= & -\frac{1}{8}\left(k^{2}+4 M^{2}\right) \frac{\partial \mathcal{B}_{1}}{\partial M}-M \mathcal{B}_{1}(k)-\frac{1}{2} M \Omega_{2}(M)+ \\
& +\frac{k_{3}^{2}}{k^{2}}\left[\frac{1}{8}\left(k^{2}+4 M^{2}\right) \frac{\partial \mathcal{B}_{1}}{\partial M}+M \mathcal{B}_{1}(k)-\frac{1}{2} M \Omega_{2}(M)\right] .
\end{aligned}
$$

Then, we notice

$$
\frac{\partial}{\partial M}\left[\frac{1}{8}\left(k^{2}+4 M^{2}\right) \mathcal{B}_{1}(k)\right]=\frac{1}{8}\left(k^{2}+4 M^{2}\right) \frac{\partial \mathcal{B}_{1}}{\partial M}+M \mathcal{B}_{1}(k)
$$

and

$$
\frac{1}{2} M \Omega_{2}(M)=-\frac{1}{4} \frac{\partial \Omega_{1}}{\partial M}
$$

We conclude that

$$
\begin{aligned}
X_{0}= & -\frac{1}{8}\left(k^{2}+4 M^{2}\right) \mathcal{B}_{1}(k)+\frac{1}{4} \Omega_{1}(M)+\frac{k_{3}^{2}}{k^{2}}\left[\frac{1}{8}\left(k^{2}+4 M^{2}\right) \mathcal{B}_{1}(k)+\frac{1}{4} \Omega_{1}(M)\right] \\
& + \text { const. }
\end{aligned}
$$

where the constant is a function of $k$ independent of $M$ and the whole expression should have no pole at $k=0$. The explicit residue of the pole is

$$
k_{3}^{2}\left(\frac{M}{16 \pi}+\frac{1}{16 \pi}(\Lambda-M)\right)
$$


Therefore, the integration constant should cancel the residue and the coefficient of $k_{3}^{2} / k^{2}$ becomes

$$
\frac{1}{8}\left(k^{2}+4 M^{2}\right) \mathcal{B}_{1}(k)+\frac{1}{4} \Omega_{1}(M)-\frac{\Lambda}{16 \pi} .
$$

To fix the complete integration constant, we can then take the $k=0$ limit. Then,

$$
X_{0}=\frac{1}{3}\left[\Omega_{1}(M)-M^{2} \Omega_{2}(M)\right]=\frac{\Lambda}{12 \pi}-\frac{M}{8 \pi} .
$$

Taking the same limit in equation (F.1), we obtain

$$
X_{0}=-\frac{1}{2} M^{2} \Omega_{2}(M)+\frac{1}{4} \Omega_{1}(M)=\frac{\Lambda}{16 \pi}-\frac{M}{8 \pi} .
$$

We thus have to add

$$
\frac{\Lambda}{48 \pi} \text {. }
$$

The complete expression reads

$$
\begin{aligned}
X_{0}= & -\frac{1}{8}\left(k^{2}+4 M^{2}\right) \mathcal{B}_{1}(k)+\frac{1}{4} \Omega_{1}(M)+\frac{\Lambda}{48 \pi} \\
& +\frac{k_{3}^{2}}{k^{2}}\left[\frac{1}{8}\left(k^{2}+4 M^{2}\right) \mathcal{B}_{1}(k)+\frac{1}{4} \Omega_{1}(M)-\frac{\Lambda}{16 \pi}\right] .
\end{aligned}
$$

\section{G Other current components and WT identities}

We have only considered the $J_{3}$ component of the current. Here, we give a few details over the three-point vertex function

$$
\tilde{\Gamma}_{\mu}(k ; p-k / 2, p+k / 2) \equiv\left\langle\tilde{J}_{\mu}(k) \psi(p-k / 2) \bar{\psi}(p+k / 2)\right\rangle
$$

where $J_{\mu}$ is defined as in eq. (8.1).

First, we can write a WT identity that relation the conserved current to the fermion inverse two-point function. It reads

$$
k_{\mu} \tilde{\Gamma}_{\mu}(k ; p-k / 2, p+k / 2)=\tilde{\Gamma}^{(2)}(p-k / 2)-\tilde{\Gamma}^{(2)}(p+k / 2) .
$$

In the large $N$ limit we know the right hand side exactly. From eq. (3.40),

$$
\begin{aligned}
\tilde{\Gamma}^{(2)}(p-k / 2)-\tilde{\Gamma}^{(2)}(p+k / 2)= & -U\left(p_{3}-k_{3} / 2\right)[i(\not p-\not k / 2)+M] U\left(p_{3}-k_{3} / 2\right) \\
& +U\left(p_{3}+k_{3} / 2\right)[i(\not p+\not k / 2)+M] U\left(p_{3}+k_{3} / 2\right) .
\end{aligned}
$$

Taken into account the explicit form (3.38) of $U$ and after a simple commutation, we obtain

$$
\begin{aligned}
& \tilde{\Gamma}^{(2)}(p-k / 2)-\tilde{\Gamma}^{(2)}(p+k / 2)=i k_{1} \sigma_{1}+i k_{2} \sigma_{2} \\
& \quad-U^{2}\left(p_{3}-k_{3} / 2\right)\left[i\left(p_{3}-k_{3} / 2\right) \sigma_{3}+M\right]+U^{2}\left(p_{3}+k_{3} / 2\right)\left[i\left(p_{3}+k_{3} / 2\right) \sigma_{3}+M\right] .
\end{aligned}
$$

One verifies then that $\tilde{\Gamma}_{\mu}$ has the simple form

$$
\tilde{\Gamma}_{\mu}=i \sigma_{\mu}+E_{\mu} \mathbf{1}+i F_{\mu} \sigma_{3},
$$

which is consistent with the WT identity.

Moreover, the quantities $\tilde{\Gamma}_{\mu}-i \sigma_{\mu}$, since they are only linear combinations of the matrices 1 and $\sigma_{3}$, all satisfy an integral equation of the form (5.31) but with different inhomogeneous terms. 
Open Access. This article is distributed under the terms of the Creative Commons Attribution License (CC-BY 4.0), which permits any use, distribution and reproduction in any medium, provided the original author(s) and source are credited.

\section{References}

[1] I.R. Klebanov and A.M. Polyakov, AdS dual of the critical $O(N)$ vector model, Phys. Lett. B 550 (2002) 213 [hep-th/0210114] [inSPIRE].

[2] E. Sezgin and P. Sundell, Massless higher spins and holography, Nucl. Phys. B 644 (2002) 303 [Erratum ibid. B 660 (2003) 403] [hep-th/0205131] [INSPIRE].

[3] E. Sezgin and P. Sundell, Holography in $4 D$ (super) higher spin theories and a test via cubic scalar couplings, JHEP 07 (2005) 044 [hep-th/0305040] [INSPIRE].

[4] R.G. Leigh and A.C. Petkou, Holography of the $N=1$ higher spin theory on AdS $S_{4}$, JHEP 06 (2003) 011 [hep-th/0304217] [INSPIRE].

[5] E.S. Fradkin and M.A. Vasiliev, On the gravitational interaction of massless higher spin fields, Phys. Lett. B 189 (1987) 89 [inSPIRE].

[6] M.A. Vasiliev, Higher spin gauge theories: star product and AdS space, in The many faces of the superworld, M.A. Shifman ed., World Scientific Singapore (2000), pg. 533 [hep-th/9910096] [INSPIRE].

[7] S. Giombi and X. Yin, Higher spin gauge theory and holography: the three-point functions, JHEP 09 (2010) 115 [arXiv:0912.3462] [INSPIRE].

[8] S. Giombi and X. Yin, Higher spins in AdS and twistorial holography, JHEP 04 (2011) 086 [arXiv: 1004.3736] [INSPIRE].

[9] S. Giombi and X. Yin, On higher spin gauge theory and the critical $O(N)$ model, Phys. Rev. D 85 (2012) 086005 [arXiv:1105.4011] [INSPIRE].

[10] S. Giombi, S. Prakash and X. Yin, A note on CFT correlators in three dimensions, JHEP 07 (2013) 105 [arXiv: 1104.4317] [INSPIRE].

[11] S. Giombi et al., Chern-Simons theory with vector fermion matter, Eur. Phys. J. C 72 (2012) 2112 [arXiv:1110.4386] [INSPIRE].

[12] O. Aharony, G. Gur-Ari and R. Yacoby, $D=3$ bosonic vector models coupled to Chern-Simons gauge theories, JHEP 03 (2012) 037 [arXiv:1110.4382] [INSPIRE].

[13] S. Jain, S.P. Trivedi, S.R. Wadia and S. Yokoyama, Supersymmetric Chern-Simons theories with vector matter, JHEP 10 (2012) 194 [arXiv:1207.4750] [INSPIRE].

[14] O. Aharony, G. Gur-Ari and R. Yacoby, Correlation functions of large- $N$ Chern-Simons-matter theories and bosonization in three dimensions, JHEP 12 (2012) 028 [arXiv: 1207.4593] [INSPIRE].

[15] G. Gur-Ari and R. Yacoby, Correlators of large-N fermionic Chern-Simons vector models, JHEP 02 (2013) 150 [arXiv:1211.1866] [INSPIRE].

[16] O. Aharony, S. Giombi, G. Gur-Ari, J. Maldacena and R. Yacoby, The thermal free energy in large-N Chern-Simons-matter theories, JHEP 03 (2013) 121 [arXiv:1211.4843] [INSPIRE]. 
[17] M. Moshe and J. Zinn-Justin, Quantum field theory in the large- $N$ limit: a review, Phys. Rept. 385 (2003) 69 [hep-th/0306133] [INSPIRE].

[18] W.A. Bardeen, M. Moshe and M. Bander, Spontaneous breaking of scale invariance and the ultraviolet fixed point in $O(N)$ symmetric $\phi^{6}$ in three-dimensions theory, Phys. Rev. Lett. 52 (1984) 1188 [INSPIRE].

[19] D.J. Amit and E. Rabinovici, Breaking of scale invariance in $\phi^{6}$ theory: tricriticality and critical end points, Nucl. Phys. B 257 (1985) 371 [InSPIRE].

[20] W.A. Bardeen, K. Higashijima and M. Moshe, Spontaneous breaking of scale invariance in a supersymmetric model, Nucl. Phys. B 250 (1985) 437 [INSPIRE].

[21] J. Feinberg, M. Moshe, M. Smolkin and J. Zinn-Justin, Spontaneous breaking of scale invariance and supersymmetric models at finite temperature, Int. J. Mod. Phys. A 20 (2005) 4475 [inSPIRE].

[22] W.A. Bardeen and M. Moshe, Spontaneous breaking of scale invariance in a $D=3 \mathrm{U}(N)$ model with Chern-Simons gauge fields, JHEP 06 (2014) 113 [arXiv:1402.4196] [INSPIRE].

[23] W.A. Bardeen, The massive fermion phase for the $\mathrm{U}(N)$ Chern-Simons gauge theory in $D=3$ at large-N, JHEP 10 (2014) 039 [arXiv:1404.7477] [INSPIRE].

[24] For an introduction see for example L. Alvarez-Gaumé, An introduction to anomalies, in Fundamental problems of gauge theory, Erice Italy 1985, G. Velo and A.S. Wightman eds., Plenum Press, New York U.S.A. (1986), pg. 93 [INSPIRE].

[25] J. Zinn-Justin, Chiral anomalies and topology, Lect. Notes Phys. 659 (2005) 167 [hep-th/0201220] [INSPIRE].

[26] G. 't Hooft, A planar diagram theory for strong interactions, Nucl. Phys. B 72 (1974) 461 [INSPIRE].

[27] For a review on matrix models see P. Di Francesco, P.H. Ginsparg and J. Zinn-Justin, 2D gravity and random matrices, Phys. Rept. 254 (1995) 1 [hep-th/9306153] [INSPIRE]. 